\title{
Calculation and Visualization of Range of Motion of Hip Joint from MRI
}

\author{
Sahar Aghayan \\ Thesis submitted to the \\ Faculty of Graduate and Postdoctoral Studies \\ In partial fulfillment of the requirements for the degree \\ Master of Computer Science \\ Ottawa-Carleton Institute for Computer Science \\ School of Information Technology and Engineering \\ University of Ottawa \\ Ottawa, Ontario, Canada \\ (C) Sahar Aghayan, Ottawa, Canada, 2014
}




\begin{abstract}
Femoro-Acetabular Impingement (FAI) is a hip joint disease which affects and impairs the range of hip motion during performing activities of daily living, jogging, walking, or climbing stairs due to the bony abnormalities of the joint. Ballet dancers and athletes (e.g. gymnasts and hockey players) put their hips at the risk of FAI by extremely moving the hip mainly by excessively rotating the joint.

In this research, we introduce a visualization system which helps surgeons to analyze the range of hip motions as well as to have a better communication with patients. These goals are achieved by presenting three dimensional (3D) visualizations of motion envelope by examining the maximum possible rotation of the digital hip bones. Our computer simulation system estimates, analyzes and visualizes the maximum hip range of motion (ROM) for the constructed 3D bone models that are extracted from Magnetic Resonance Images (MRI) after segmenting the bones. These tasks are accomplished by first calculating Hip Joint Center (HJC) which is center of rotation of femoral head on the 3D segmented MRI models followed by simulating hip motions with examining impingement between the femur and the acetabulum using our collision detection system.
\end{abstract}

In our collision detection system, surfaces of femoral head and acetabulum bones are sampled in the spherical coordinates based on rasterization and interpolation. Then, the distance between the femoral head and acetabulum are computed to prevent impingement between them. The maximum motion degree of femur bone within depression of acetabulum in every direction 
during the digital simulation shows the ROMs of the inputted MRI of the hip joint. Six primary plane motions (flexion/extension, abduction/adduction and internal/external rotation) as well as various combinations of these motions (maximum rotation of the hip between every two rotational movements) and successive movements (maximum rotational movement of the hip per another rotational movement) are simulated and analyzed along with 3D visualization of estimated range of these motions.

Generally, the ROM differs by some factors such as age, gender, ethnicity, and geographic location. For instance, newborns up to age two have considerably greater motion in hip flexion and hip abduction than adults. Our system by 3D visualization of motion envelope will provide a platform to understand quicker and better the effect of bony morphology of the hip joint on the possible ROM.

We also examine the long-standing question about moving center of rotation related to ROM. We found out the ROM becomes bigger especially when the center moves outward to the direction of acetabulum axis.

This thesis does not consider the effect of muscle and other surrounding connective tissue on the hip ROM since they can be altered significantly by physical training to show the potential of maximum ROM. For example a ballerina has a bigger ROM leading a bigger motion envelope compared with non-dancers. Hence we visualize the range of joint motions and their envelopes that are obtained from the osseous anatomy of the hip joint. The osseous anatomy of the joint is the most fundamental and permanent factor of ROM which indicates the maximum motion that the joint can achieve if the muscle and other connective tissues are perfectly trained. 


\section{Acknowledgments}

I would like to take this opportunity to express my profound gratitude and deep regards to my guide, Prof. WonSook Lee for her exemplary understanding, guidance, support and constant encouragement throughout the course of this thesis.

I also take this opportunity to express a deep sense of gratitude to my sister, Niloofar, for her cordial support and valuable information, which helped me in completing this task through various stages.

I would like to thank Dr. Kawan Rakhra and Dr. Paul Beaulé (Division of Orthopaedic Surgery, Faculty of Medicine, University of Ottawa) for the valuable knowledge and comments provided by them on the medical aspects. I am grateful for their cooperation during the period of my research study.

My special thanks go to my dear friend, Sasha, who constantly encouraged me with his love, never-ending support and all the laughter he gave me throughout all those years, which were not always so easy.

I also would like to thank my friend, Mahbubeh for being always here for me when I needed a good laughter or a piece of advice.

Last but not least, I owe my deepest gratitude to my parents for their endless love and for having faith in me, encouraging me in every decision I made in my life and always believing in my choices that led me to this place. 


\section{Dedication}

\section{To my}

Mother and Father

for their endless love, support and encouragement 


\section{Table of Contents}

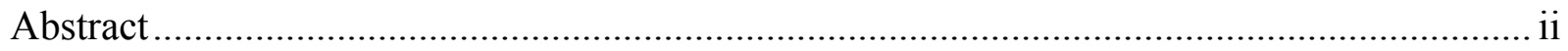

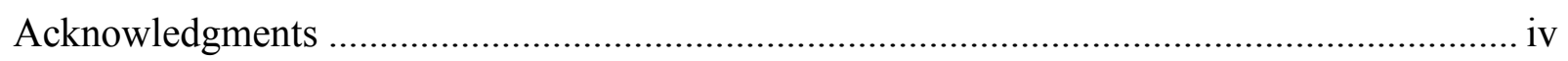

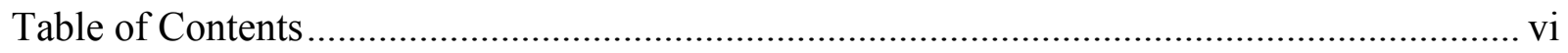

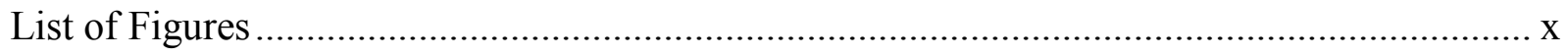

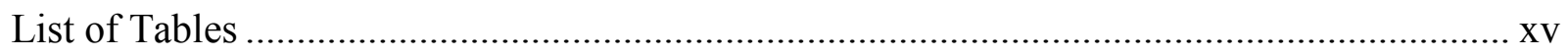

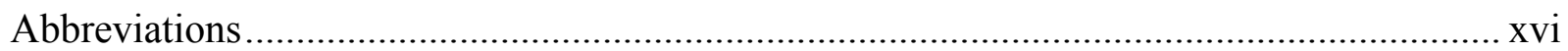

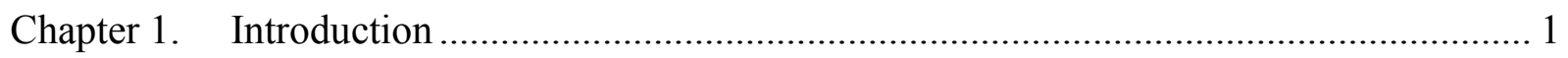

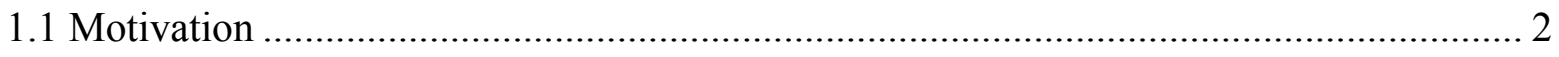





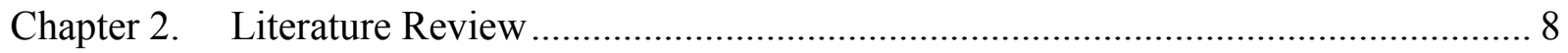

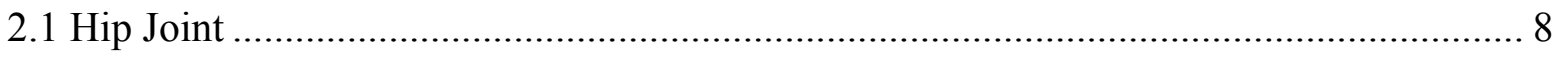

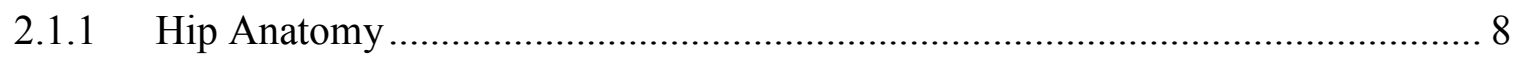

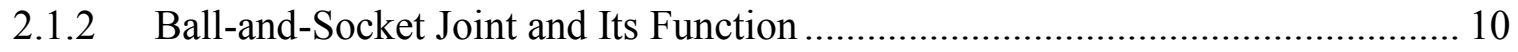

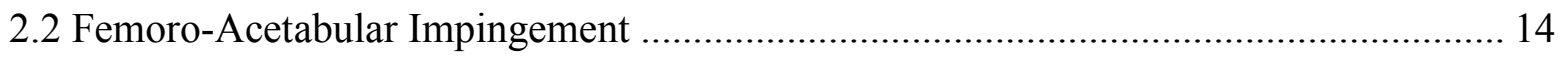

2.2.1 Physical Examination of the Hip in Real-Life ............................................ 15 
2.2.2 Treatments of Femoro-Acetabular Impingement ........................................ 16

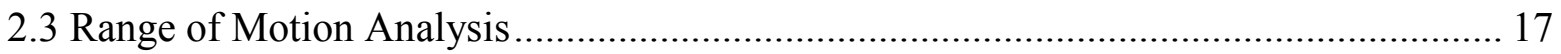

2.3.1 Hip Joint Center ................................................................................... 18

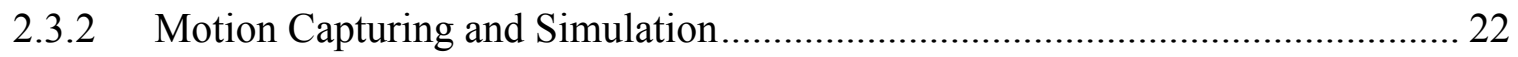

2.3.3 Computer Simulation of Impingement Detection ........................................ 24

2.4 Collision Detection Methods ............................................................................... 25

2.4.1 Bounding Volume Hierarchy ......................................................................... 25

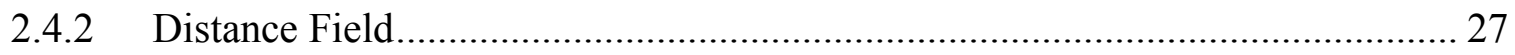



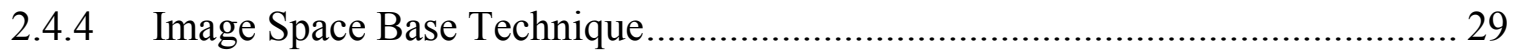

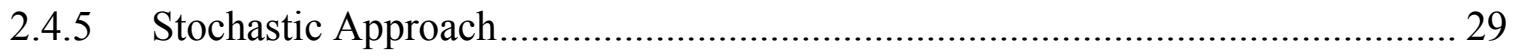





3.1 Construction of 3D Hip Bones ........................................................................... 34

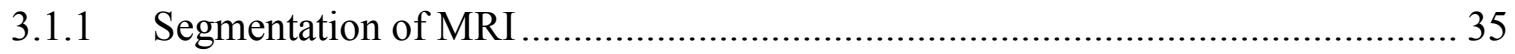

3.1.2 Construction of 3D Surface Models........................................................... 37

3.2 Hip Joint Center Calculation .............................................................................. 38

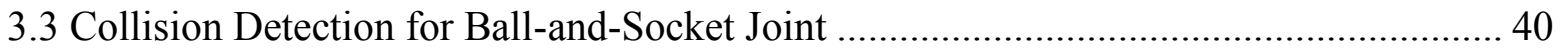




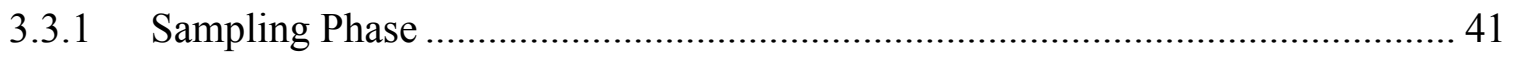

3.3.2 Collision Detection Phase …………………............................................. 50

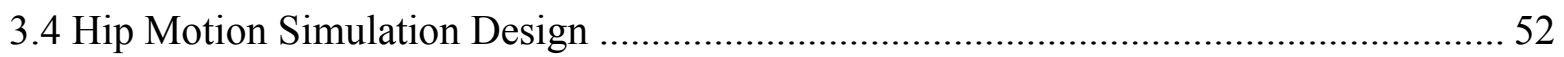

3.4.1 Simulation of Rotational Movements …………............................................. 53

3.4.2 Moving Hip Joint Center........................................................................... 58

Chapter 4. Result and Visualization of Range of Motion ...................................................... 61

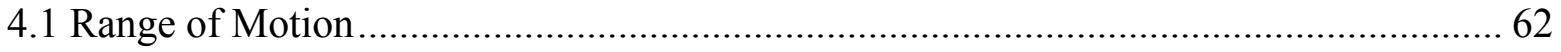





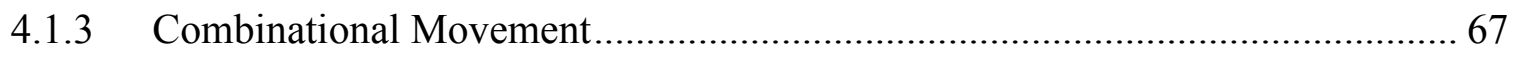

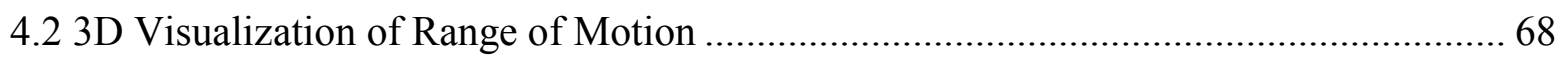

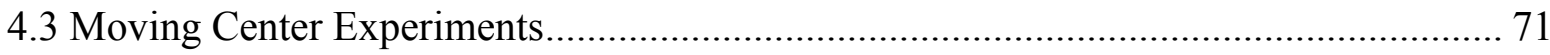



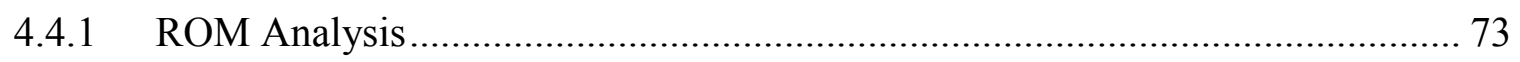

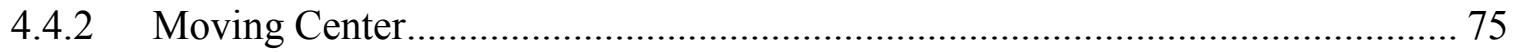

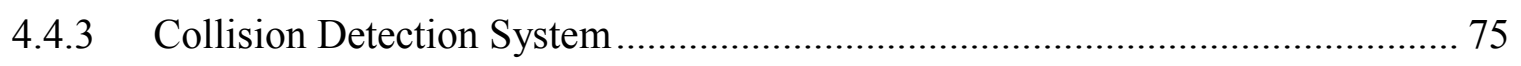



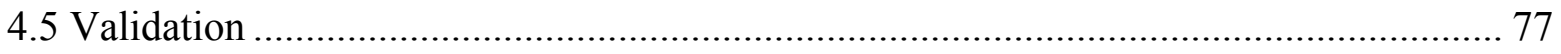

viii 
Chapter 5. Conclusion......................................................................................... 81



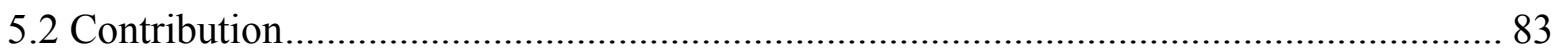

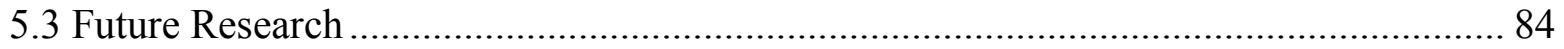

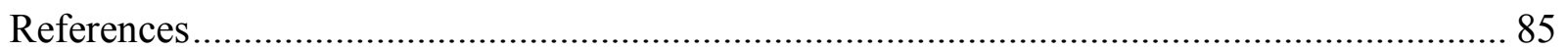






\section{List of Figures}

Figure 1.1: Process pipeline of our proposed system. .................................................. 6

Figure 2.1: Anterior view of bony feature of hip joint (reproduced from [8]). ....................... 8

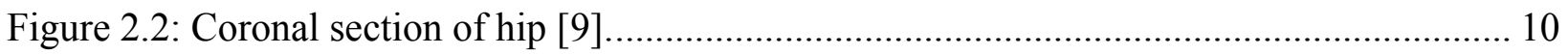

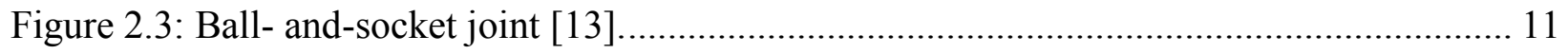

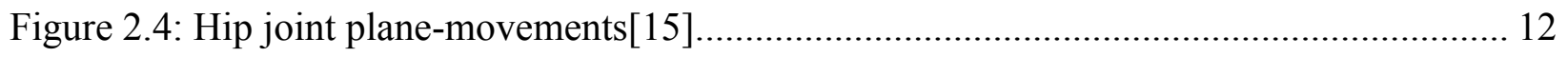

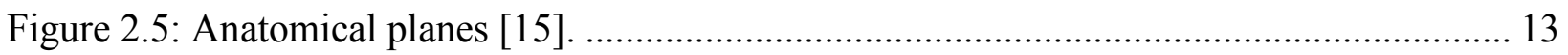

Figure 2.6: (A) Normal morphology of the hip joint without impingement when it is flexed in (B). (C) Cam deformity in a neutral pose when anterosuperior impingement occurred when the femur is flexed in (D). (E) A pincer deformity in a neutral position, where anterosuperior impingement occurred when the femur is flexed in $(F)$. (G) Combination of a cam deformity and a pincer deformity where cause the anterosuperior impingement and the postero-inferior impingement as shown in $(\mathrm{H})$. The excess bones are shown in gray color and the impingement



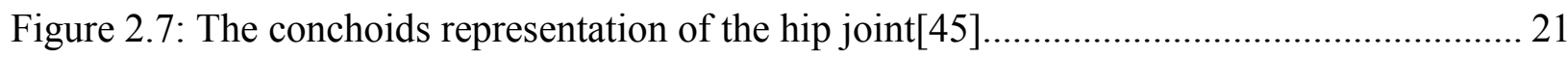

Figure 3.1: High-level view of our motion simulation and visualization system..................... 33

Figure 3.2: a-c) segmented MR images in axial, sagittal and coronal planes from ITK-SNAP. d) 3D constructed femur (shown in red) and pelvis (shown in blue) models. ............................. 36 
Figure 3.3: Cross-section of upper femur that displays compact and spongy bones distributions $[70]$. 37

Figure 3.4: a) Point cloud constructed from segmented MRI of right femur. b) Result of 3D mesh reconstruction and geometry correction proposed by Attene [72] c) 3D model surface after reducing its complexity. 38

Figure 3.5: Result of Hough transform in anterior, right lateral, posterior and left lateral views of a right femur model (respectively from left to right). 39

Figure 3.6:2D sketch of our collision detection algorithm for ball-and socket joint from left to right: The surfaces of the hip bones are sampled according to HJC (red dot) and checked for collision. If the distance (d) between samples of two surfaces is less than or equal zero, there is collision between two surfaces otherwise no collision is detected. 40

Figure 3.7: Calculation of acetabulum center: a) four markers (red dots) on acetabulum is set up, three on the rim and one on its depth. b,c) Result of spherical approximation of acetabulum..... 42

Figure 3.8: Illustration of bounding sphere (red color) for the acetabulum that is localized on the center of acetabulum with radius $\mathrm{R}^{\prime}$ ( $\mathrm{R}$ is the radius of the sphere fitted to quasi-spherical portion of the acetabulum). 43

Figure 3.9: Sample a random point in triangle 45

Figure 3.10: Random generation of sample points on femoral head surface proportionate to each triangle area of mesh. 45

Figure 3.11:2D conceptual illustration of surface sampling (sampling precision of $0.05^{\circ}$ ): red dots represent HJC as center of spherical coordinate. Dash lines demonstrate the sample points xi 
distance from the center. (a) Black dots represent instances of sampling points on the femoral head surface. (b) Black squares represent the generated sample points on surface of acetabulum.

Figure 3.12: Spherical coordinate system. 48

Figure 3.13: Triangle rasterization: a bounding box (red box) is fitted around each triangle in order to iteratively generate sample points by using rasterization and interpolation.

Figure 3.14: 2D conceptual representation of collision detection procedure: (a) samples point of each femoral head (black dots) and acetabulum (black square) surfaces are represented from HJC (red dot). (b) Distance difference between sample points of both models surfaces (black arrows) is computed...... 51

Figure 3.15: Illustration of coordinate system of 3D proximal femur and pelvis bones based on HJC. 54

Figure 3.16: Six main plane-movements of the hip joint: a)flexion b)extension, c)abduction, d)adduction, e)internal rotation, f)external rotation. 55

Figure 3.17 a-f) Illustration of successive movement; flexion movement per abduction movement. a) Hip in neutral pose where the abduction degree is zero. $b, d$ and $f$ show the maximum flexion per abduction movement of a $\left(0^{\circ}\right)$, c $\left(20^{\circ}\right)$, e $\left(40^{\circ}\right)$, respectively. 56

Figure 3.18. a-e) Illustration of combinational movement of the hip joint that shows the maximum rotational movement between flexion (b) and abduction (e) movement, around 12 to 3 clock hours hand ( "a" shows the hip in neutral pose) 57 
Figure 3.19: Illustration of a projected clock on the transverse plane (the black square) which is passing through HJC (noted that center of clock is placed at the HJC). The left snapshot is topview and the right is front-view. The axis $\mathrm{Z}$ is the front of body and the axis $\mathrm{X}$ is inward. 58

Figure 3.20: Illustration of center of the acetabulum (indicated as COA in red) compare with the HJC or center of the femoral head (indicated as COF in black). 60

Figure 3.21: Illustration of moving the HJC a)outward and b)inward the acetabulum. 60

Figure 4.1: Illustration of impingement zone (red color) during flexion movement. 63

Figure 4.2: Illustration of impingement zone (red color) during abduction movement. 63

Figure 4.3: Maximum rotational angles $\left(^{\circ}\right)$ of flexion and extension in diverse abduction degrees.

Figure 4.4: Maximum rotational angles $\left(^{\circ}\right)$ of flexion and extension in diverse adduction degrees. 65

Figure 4.5: Maximum flexion rotational angles (degrees) of hip joint in degrees of external and internal rotations. 66

Figure 4.6: Maximum rotational angles $\left({ }^{\circ}\right)$ of hip joint around clock hours hand when the clock face is projected on the transverse plane that is passing through the HJC (center of clock is placed at the HJC). 68

Figure 4.7: Envelope visualization of Figure 4.6 which represents hip combinational movements. Top row is colored representation of this envelope and bottom row is wired representation of the envelope, from different views. 
Figure 4.8: Envelope visualization of Figure 4.3 representing hip successive movements; abduction with flexion and extension. Blue envelope represents the extendibility of the hip joint per abduction movement while the red envelope represents the flexibility of abducted joint (yellow line shows when the hip is abducted only) 70

Figure 4.9: Comparison between the effects of moving center on the maximum ROMs. .......... 72

Figure 4.10: color scale of the difference from femoral head surface and fitted sphere. 78 


\section{List of Tables}

Table 2.1: Normal values for the range of motion of the hip joints which are measured in real-life [14]. 11

Table 2.2:Difference in mean active range of motion (in degree) for ages 25-39 years compared with ages 60-74 years by sex and race groups which are measured in real-life [7]. 12



Table 3.1: Anatomical reference axes and planes of hip joint rotational movements. 54

Table 4.1: Our result of rotational degrees for six extreme plane-movements of right hip around a fixed HJC. 62

Table 4.2: Maximum ROMs of six plane-movements of hip joint when the HJC is moved to the acetabulum center, outward the acetabulum depression compared with fixed HJC (center of femoral head). 71

Table 4.3: Maximum ROMs of six plane-movements of hip joint when the HJC is moved to the acetabulum center, inward the acetabulum depression compared with fixed HJC. 72

Table 4.4: Quantitative errors obtained from difference between femoral head surface and fitted sphere. 78

Table 4.5: Performance analysis of our collision detection algorithm. 79

Table 4.6:Evaluation of our computed ROMs with the normal ranges [14] and mean active range of motion for white men with ages 25-39 years [7] 80 


\section{Abbreviations}

2D Two Dimensional

3D Three Dimensional

AABB Axis Aligned Bounding Box

ADF Adaptively Distance Field

BVH Bounding Volume Hierarchy

CT Computed Tomography

DICOM Digital Imaging and Communication in Medicine

DOP Discrete Orientation Polytope

DOF Degree Of Freedom

FAI Femoro-Acetabular Impingement

HJC Hip Joint Center

LUT Look-Up Table

MRI Magnetic Resonance Imaging

OBB Oriented Bounding Box

PSIS Pubic Symphysis Iliac Spine

PSIS Anterior Superior Iliac Spine

ROM Range of Motion

RSID Rapid Spherical Impingement Detection

SL Sampling List

SST Spherical Sliding Table 


\section{Chapter 1. Introduction}

A massive amount of factors including biochemical, genetic, and common morphological abnormalities may lead to early Hip Osteoarthritis (OA) [1] [2] [3] especially in young and active patients such as ballet dancers and athletes. Femoro-Acetabular Impingement (FAI) is characterized as an anatomical morphology of the acetabulum and the proximal femur which causes abnormal contact at the hip joint and attenuates articular cartilage thickness. Some investigation shows that some movement increase load and consequently stress in the joint [4]. Indeed, sporting activities that require repetitive and extreme movements (e.g. dancing and gymnastic exercises) cause degeneration of labrum and frequent impingement between bones. Bones rub against each other causing stiffness and pain. Consequently, FAI impairs hip joint and leads to limitation of movement and declines range of motion (ROM) [5] [6].

ROM is defined as the permitted motion of the hip joint and it is obtained from impingementfree motion. A number of anatomical (e.g. gender, age and ethnicity) and training-related (activity level) factors affects the maximum possible ROM [7]. For example, hip rotations decrease with increasing age[7].

In order to diagnose FAI, different clinical evaluation of the hip joint is employed before any operation, which includes physical hip examinations, magnetic resonance imaging (MRI) or computed tomography (CT) examinations and morphological analysis of bony structures [7]. Clinical hip examinations are mainly aimed to localize pain and determine joint ROM. During the examinations, different hip rotational movements are executed, for instance, internal rotation degree for flexed and adducted hip in a patient with positive impingement test are assessed. On 
the other hand, surgical operation of hip joint involves soft tissues and osseous repairs with the purpose of improvement in the clearness for hip motion. Since the hip operations are highly risky, it is essential that surgeon has an effective vision about the range of joint motion before operation to know exactly about the surgery strategy and to minimize the risk of miss-operation.

\subsection{Motivation}

A typical way of motion study is based on markers placement and motion capture systems in real-life. However, it has disadvantages of being dependent and sensitive to the markers placement, skin cloth artifacts and size of the markers. In addition, it requires a specialized experimental room setting for motion capture and it is difficult and painful to obtain maximal motions. Furthermore, it is difficult to isolate the effect of bone outlines out of anatomical factors such as muscles, tendon and other connective tissues which are merged in motion capture in reallife. Also various factors such as warm up and stretching exercise affect the acquired motion capture data resulting greater ROM.

Thereby, we are motivated to develop a computer-assisted simulation system to digitally simulate and analyze maximum hip rotational movements from bony morphologies of provided MRI data. In other words, since the aforementioned anatomical factors (muscles and connective tissues) are variable and can be altered by doing physical training, we are motivated to digitally simulate maximum movements only from bony structures of the hip.

Also, a computer-assisted technology can help to better quantify hip kinematics and kinetics in function of hip joint bony morphologies, with the aim of a better understanding of degenerative hip disease etiologies in active and young patients. Thus, we are motivated to develop a three 
dimensional (3D) visualization system to simulate hip joint motion and estimate the maximum ROM while a collision detection algorithm is used to restrict hip movement to prevent impingement between the osseous anatomy of the proximal femur and the acetabulum. Additionally, since extreme ranges of motion are evaluated mainly from bone morphologies, path and possible range of hip motion that are restricted by bones impingements can be estimated and analyzed through a computerize simulation. Quantification of the maximum attainable ROM is much painless and effortless With the use of digital simulation and visualization technologies. This also gives us the chance to investigate hip bony morphological constraints to determine whether a person (specifically a dancer) has the potential to do some extreme motions and exercises or not.

A 3D simulation and visualization of patient's hip joint and its ROM can help surgeon to have a better communication to patients and it will provide a valuable insight into the understanding of hip pathology. A 3D visualization of motion envelope provides a quick and intuitive understanding of the effect of FAI on the maximum possible range of hip motion. Therefore, the FAI patient would be able to broadly understand why she/he needs to have the surgery and how this operation can improve her/his joint mobility.

It can also be used for non-FAI patients:

(i) To advise athletes, how big their maximum possible hip movement is to prevent impingement between the osseous anatomy of the proximal femur and the acetabulum by pushing too hard.

(ii) To encourage non-athlete people to do more exercise to train their muscles by determining the maximum ROMs achievable with their hip bone shapes. 
Moreover, it had been discussed that the hip joint does not move around a fixed center of rotation, rather movement of the hip joint is associated with a firm amount of translation. Therefore, through this research, we examine the possibility of the ROM improvement by moving the center of rotation in direction of acetabulum axes. Our digital simulation system allows us to do this experiment.

\subsection{Overview of the System}

The system is composed of several steps to achieve our goal as shown in Figure 1.1.

- Segmentation of MRI: MRI of patients are collected and manually segmented by using ITK SNAP software to obtain bony structure of the hip with verification from medical doctors.

- 3D surface construction of the femur and the pelvis bones: 3D mesh models of the proximal femur and the pelvis are constructed and refined from segmented MRI of the hip joint.

- Calculation of HJC: The center of 3D proximal femur is calculated based on the best sphere fitted to its head and it is considered as HJC.

- Computer simulation of motion: Based on bony morphological constraints of the hip joint, movement of the hip around a fixed HJC is digitally simulated. The hip joint motions are simulated digitally as rotational movement in three different planes, flexion/extension in sagittal plane, abduction/adduction in frontal plane, and internal/external rotation in transverse plane. The combination of these motions and successive motions are also constructed for the hip joint. The movement of the hip in 
every direction is restricted by a collision detection algorithm associated with bones morphologies of the joint. Collision detection algorithm is based on sampling of the faces of triangulated mesh models (the femoral head and the acetabulum) and comparison of distance between sampled surfaces. In addition, the HJC is moved in direction of the acetabulum axes and aforementioned movements are conducted for the new center with the aim of obtaining greater ROMs.

- ROM analysis and visualization: The maximum degree of rotation for every motion of the hip is calculated and analyzed. The ROM and its envelope is visualized for every conducted motion. Moreover, the calculated ranges from moving center are analyzed to determine at which position hip joint reaches the maximum motion. 


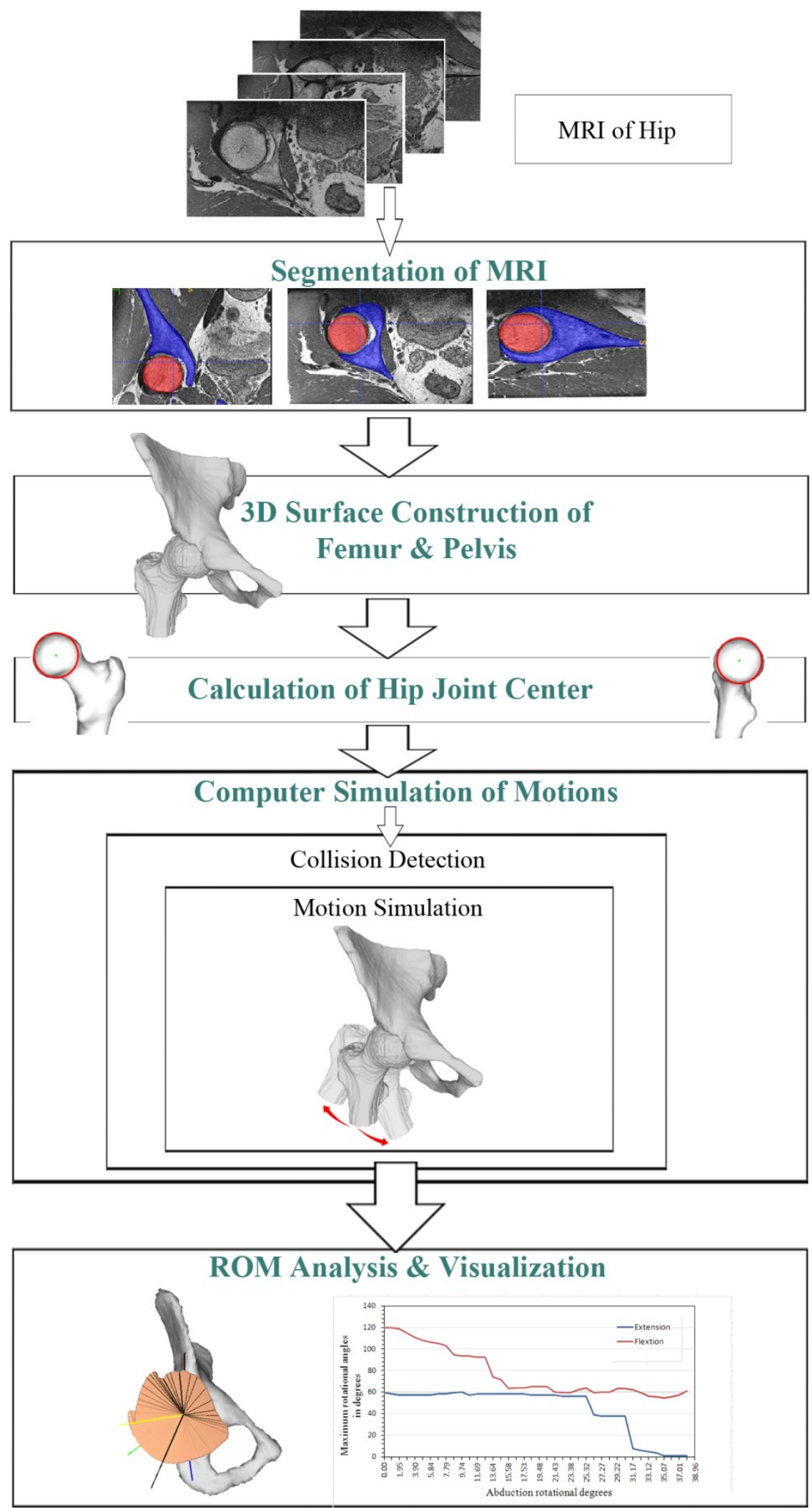

Figure 1.1: Process pipeline of our proposed system. 


\subsection{Overview of the Thesis}

The rest of this thesis is organized as follow:

- In chapter 2, we have detailed the anatomy and functionality of the hip joint; the literature review on FAI, methodologies for hip motion analysis based on different methodologies for the hip joint center calculation and collision detection, details on the algorithms and studies conducted for simulation and visualization of hip ROM.

- Chapter 3 outlines the methodologies used for this research, experiments performed along with sample result.

- In chapter 4, we describe the experimental setup and implementation detail, exhibit and analyze the results and also we demonstrate the sample visualization result of the system. This chapter also discusses the system evaluation results and limitation of the current work.

- Chapter 5 marks our conclusion along with our contribution, potential and future direction of work. 


\section{Chapter 2. Literature Review}

\subsection{Hip Joint}

\subsubsection{Hip Anatomy}

The hip joint is a ball and socket joint which is designed to be a stable, weight bearing joint. It is located where the thigh bone (femur) meets the pelvic bone (Figure 2.1).

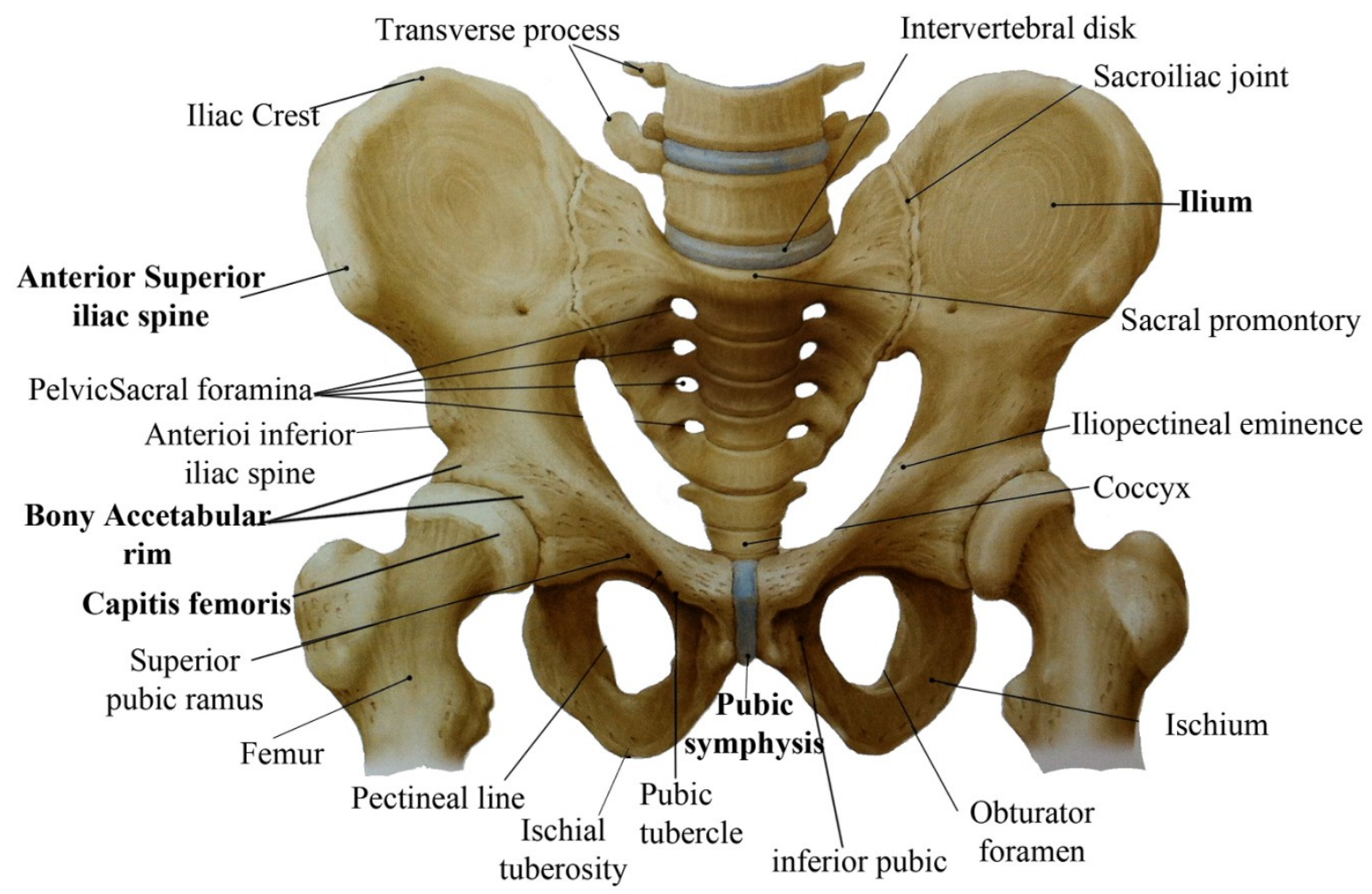

Figure 2.1: Anterior view of bony feature of hip joint (reproduced from [8]).

Femur is labeled as the longest and strongest bone [9] in the human body which its features consist of a rounded head with the indentation of fovea capitis, greater and lesser trochanters, 
and medial and lateral condyles [8]. The ball-shaped head of the femur is attached to the femoral shaft which is the main tubular part of femur by the femoral neck [10]. Femoral neck varies in length depending on body size and gives a hip joint a wide range of movement [10]. The upper segment (head) of the femur fits tightly inside the cavity in the pelvic bone that forms the socket, also known as the acetabulum [11].

Both the femoral head and the acetabulum are covered with a smooth, cushioning layer called articular cartilage. The articular cartilage reduces friction during joint movement. While the cartilage covers the entire head of the femur, the acetabulum cartilage is in the shape of a horseshoe with a cup_like depression (fossa) in the center of the socket [8]. This fossa contains soft tissue and a ligament connecting the femoral head to the socket. Although not clearly delineated, "the ligament of femoral head (ligamentum capitis femoris) would extend within the acetabulum fossa to the fovea capitis femoris" [9] (Figure 2.2). The ligament of femoral head has "little function other than protecting and transmitting the major arterial supply to the femoral head" [9]. Besides, the socket has a rim of hard fibrocartilage called the labrum. The labrum acts as a gasket, sealing in joint fluid and maintaining lubrication and nutrition. It "plays a role in normal joint development and in distribution of forces around the joint" [10].

In addition, numerous tendons surround the hip, as well as muscles. Generally, the internal structure of the bones can be classified by the two types of osseous tissue; compact and spongy bone. Compact bone (Cortical bone) is dense and homogeneous shaping the outer layer of bone. It provides maximum strength for the bones with minimal weight. Spongy bone (Cancellous) is composed of small needlelike pieces of bone called trabeculae which gives strength in order to tensile forces while minimizing weight. 


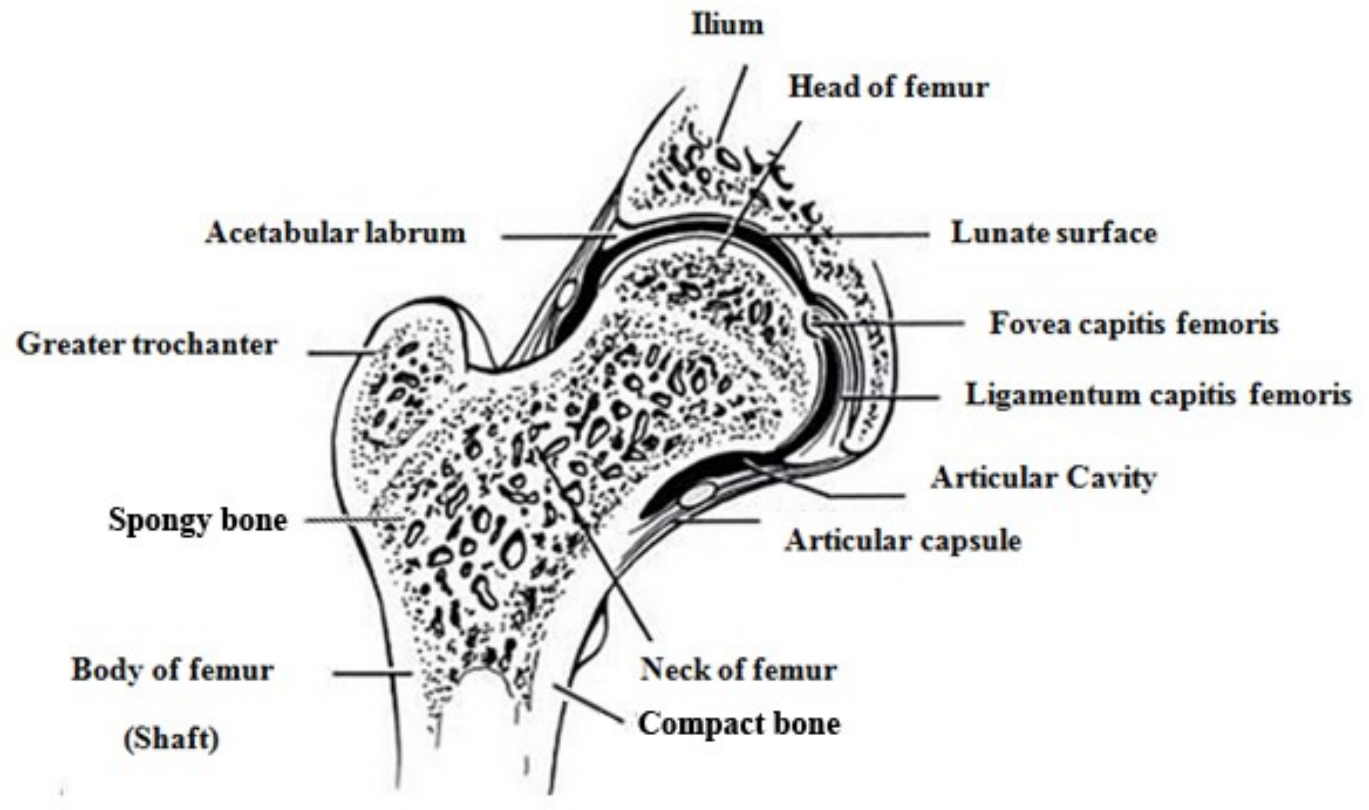

Figure 2.2: Coronal section of hip [9].

\subsubsection{Ball-and-Socket Joint and Its Function}

Ball-and-socket joint comprises the most degree of freedom (DOF), hence it is the most mobile of the purely rotational joints [12]. Three independent rotational degrees of freedom are characterized for hip joint as an enarthrodial ball-and-socket joint due to articulated rigid body segments; the femoral head and the acetabulum (Figure 2.3). In general each specific joint has a normal range of motion that is expressed in degree, constrained by joint limit (Table 2.1). Range of motion (ROM) refers to the distance and direction which a joint can move to its full potential from its neutral pose. Hip joint undergoes movement in an assortment of motion axes that allow flexion/extension in sagittal plane, abduction/adduction in frontal plane, and internal/external rotation in transverse plane (Figure 2.5). It is important to note that ROM varies due to age, gender, ethnicity, and geographic location [7] (Table 2.2). For example hip rotations decrease with increasing age. 

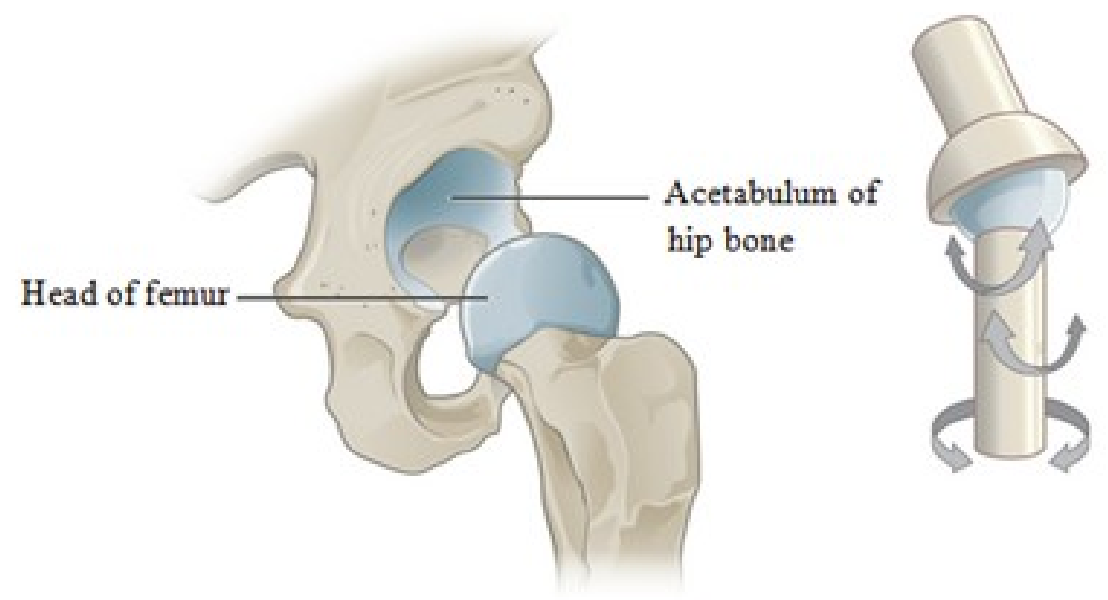

Figure 2.3: Ball- and-socket joint [13].

Table 2.1: Normal values for the range of motion of the hip joints which are measured in reallife [14].

\begin{tabular}{|c|c|}
\hline Motion & Range ( $^{\circ}$ ) \\
\hline Flexion & $0-125$ \\
\hline Extension & $115-0$ \\
\hline Abduction & $0-45$ \\
\hline Adduction & $45-0$ \\
\hline Medial (Internal) Rotation & $0-45$ \\
\hline Lateral (External) Rotation & $0-45$ \\
\hline
\end{tabular}


Table 2.2:Difference in mean active range of motion (in degree) for ages 25-39 years compared with ages 60-74 years by sex and race groups which are measured in real-life [7].

\begin{tabular}{|l|c|c|c|c|c|}
\hline \multicolumn{1}{|c|}{ Motion } & $\begin{array}{c}\text { Combined } \\
\text { Group }\end{array}$ & White Men & White Women & Black Men & Black Women \\
\hline Flexion & 122 & 123 & 123 & 115 & 116 \\
Ages 25-39 yr & 118 & 118 & 119 & 118 & 106 \\
Ages 60-74 yr & & & & & \\
Extension & 22 & 22 & 22 & 19 & 17 \\
Ages 25-39 yr & 17 & 17 & 16 & 16 & 12 \\
Ages 60-74 yr & & 46 & 44 & 41 & 38 \\
Abduction & 44 & 39 & 40 & 38 & 37 \\
Ages 25-39 yr & 39 & 34 & 33 & 32 & 27 \\
Ages 60-74 yr & 33 & 31 & 29 & 27 & 25 \\
Internal Rotation & 30 & 33 & 36 & 32 & 32 \\
Ages 25-39 yr & 34 & 27 & 32 & 27 & 28 \\
Ages 60-74 yr & 29 & & & & \\
External Rotation & & & & & \\
Ages 25-39 yr & & & & & \\
Ages 60-74 yr & & & & & \\
\hline
\end{tabular}
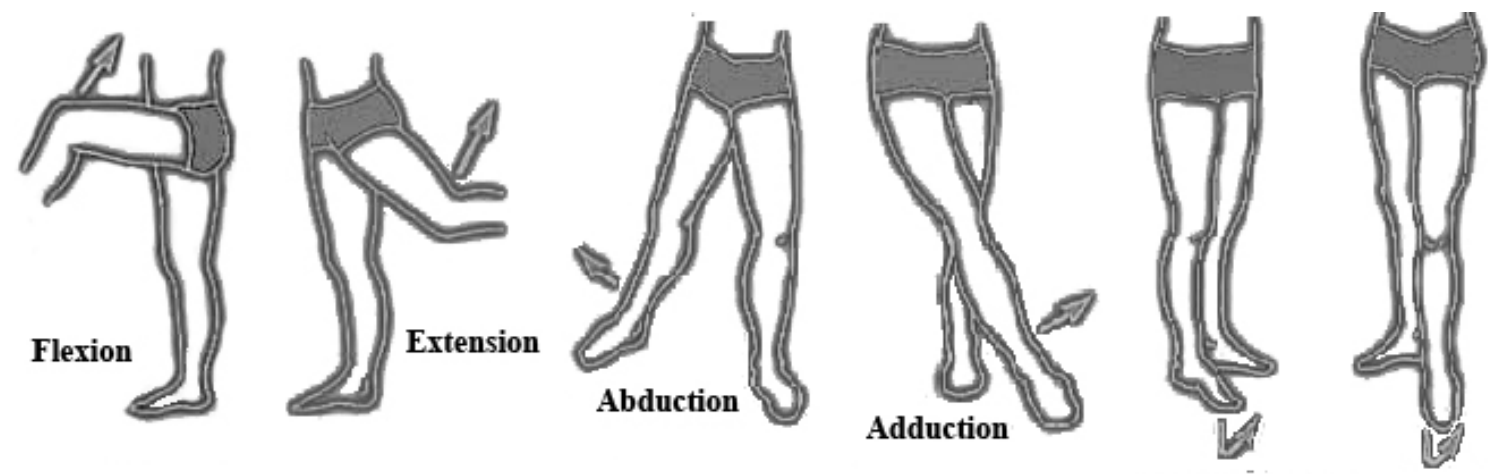

Medial Rotation

Lateral Rotation

Figure 2.4: Hip joint plane-movements[15]. 


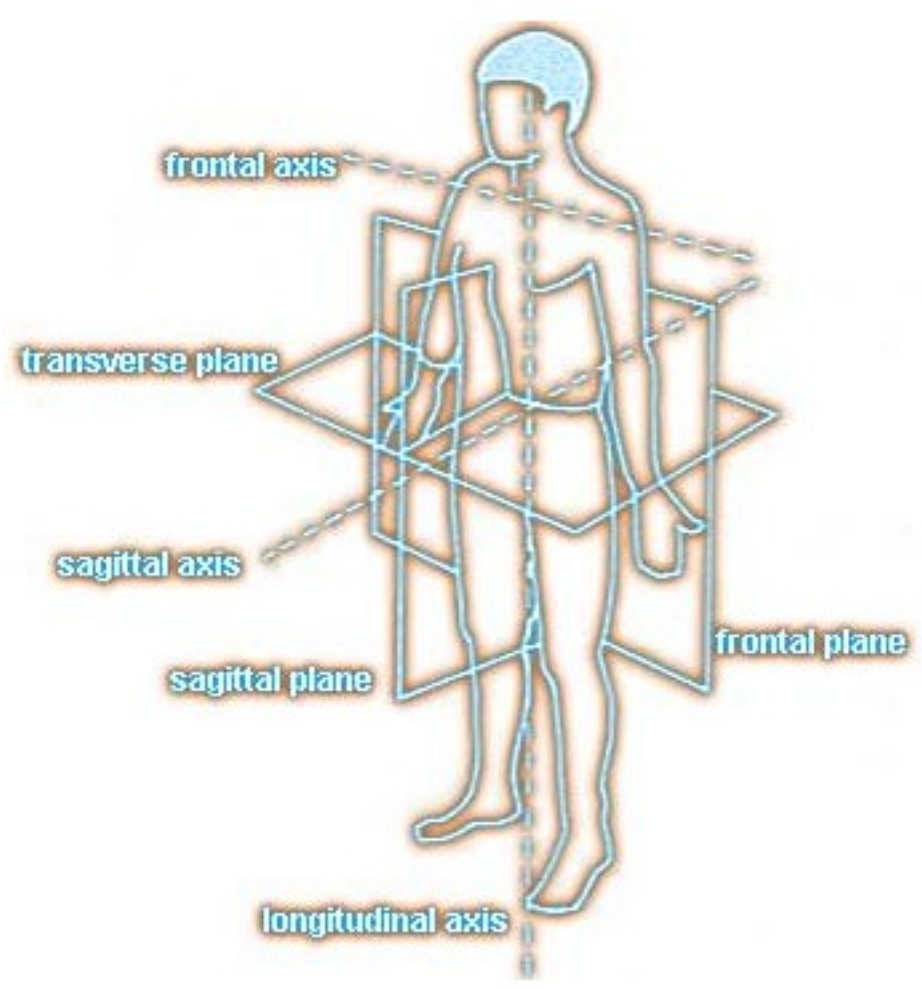

Figure 2.5: Anatomical planes [15].

Table 2.3: Anatomical terms relating to directions [11].

\begin{tabular}{|c|c|}
\hline Anatomical Terms & Directions \\
\hline Medial & Toward the middle of the body \\
\hline Lateral & Away from the middle of the body \\
\hline Proximal & Nearest to a reference point \\
\hline Inferior & Below \\
\hline Superior & Above \\
\hline Anterior & Toward the front \\
\hline Posterior & Toward the back \\
\hline
\end{tabular}




\subsection{Femoro-Acetabular Impingement}

In the context of the hip joint, the morphological abnormality of femur and acetabulum is known as Femoro-Acetabular Impingement (FAI) [3] [16] [17] [18] which occurs by either point or regional loading of the femur, typically the femoral head-neck junction against the acetabular rim [19]. In general, two types of FAI can be distinguished, pincer and cam FAI, which limit the joint rotation (Figure 2.6). Often, there is a combination of both, with one type being predominant (Figure 2.6). The pincer FAI is characterized by overcoverage of the femoral head by the acetabulum [3] or acetabulum retroversion [16]. This morphological abnormality of acetabulum increases the relative depth of the acetabulum resulting in severe contact with the femoral neck. "Continued impact of the relatively normal femoral neck against the acetabular rim leads to pincer abutment and lesions in the acetabular rim" [19]. In contrast, the cam FAI is caused by osseous bump deformity on the femoral head-neck junction [2] [3] which leads a nonspherical femoral head. During forceful motion especially flexion, acetabulum rim rubs this bumb on the femoral head neck junction [19]. This abnormal contact attenuates acetabular cartilage with outside-in abrasion or avulsion consecutively leads delamination and detachment of acetabulum labrum. Consequently, FAI provokes abnormal contact stress and potential joint impair and it leads a significant reduction in ROM, which restricts daily activities of individuals. This abnormality specifically limits hip flexion and internal rotation in $90^{\circ}$ flexion. When interviewing the patient, it is important to elicit any history of previous hip disease or hip surgery

of the patient. Also, a description of the present condition is documented. After documentation of the patient history and symptoms, a careful physical examination is critical. 


Normal joint

Figure 2.6: (A) Normal morphology of the hip joint without impingement when it is flexed in (B).

(C) Cam deformity in a neutral pose when anterosuperior impingement occurred when the femur is flexed in (D). (E) A pincer deformity in a neutral position, where anterosuperior impingement occurred when the femur is flexed in $(F)$. $(G)$ Combination of a cam deformity and a pincer deformity where cause the anterosuperior impingement and the postero-inferior impingement as shown in (H). The excess bones are shown in gray color and the impingement areas are shown in red color.

\subsubsection{Physical Examination of the Hip in Real-Life}

The physical examination [20] [7] of hip should begin with a close inspection of the patient's posture, stance, and gait followed by palpation and evaluation of abductor strength, since it commonly reveals slight weakness. Moreover, hip ROM should be assessed carefully. One 
common examination is assessment of internal rotation degree of the flexed and adducted hip when a patient has a positive impingement test with either cam or pincer FAI.

Patient is examined in supine position. In a patient with negative X-Ray radiography suspected to intra-articular impingement, the affected hip is passively flexed to ninety and leg is internally rotated and adducted. If there is abnormal contact between the anterior-superior acetabular rim and femoral neck, pain may be elicited and this reproduces the symptom of groin pain. Posterior impingement of the hip is assessed with the patient in a prone position or it may be tested by having the patient in supine position with dangling their legs off the end of an examination table. The affected leg is extended and externally rotated by the examiner (passive movement), and the opposite limb may held flexed by the patient (active movement). This provocative test produces postero-inferior impingement with deep seated groin pain.

\subsubsection{Treatments of Femoro-Acetabular Impingement}

Based on FAI severity, different kinds of surgical techniques can be employed. FAI treatments primarily aim to involve soft tissues and osseous repairs in order to improve the clearance for hip motion and alleviation of hip joint pain caused by abutment of femoral head against acetabular $\operatorname{rim}[1]$.

The standard treatment of FAI is considered as open osteochondroplasty which the femoral head is dislocated. In cam impingement, removing any non spherical osseous portion of femoral head improves the neck offset and joint rotation boundary [21]. Other treatment that applies on pincer impingement is periacetabular osteotomy which includes reducing excessive pressure of the femoral head on the rim of the acetabulum and improving instability of the femoral head within the acetabulum [22]. These improvements are accomplished by cutting the bone around 
the acetabulum in order to rotate it into a more stable, horizontal position of coverage on top of the femoral head. Moreover, Arthroscopic is another type of treatment for FAI was introduced by Sampson [23] which reattaches and excises the labral tear. It is an "edition of the open surgical procedure which follows the same steps and precautions of the open surgical technique in combination with standard techniques for arthroscopy of the central and peripheral compartment of the hip" [24].

\subsection{Range of Motion Analysis}

ROM analysis often requires determination of the HJC location and also precise detection of collision [6] [25] [26]. There are many studies in this area, which are fulfilled by the cadaveric specimens [27], mechanical linkages [28] [29], live human body [30] and 3D anatomical models which are constructed from medical data [31] [32] [33] [34] [35]. Some of these researches are aimed to analyze the effect of the cam and pincer FAI [30] on the hip kinematics and also effect

of hip joint parameters (joint center and axes of rotation) on the ROM [36]. Besides, some other researchers investigates the extreme ROMs for healthy and specific type of the hip to verify if repetitive extreme motion can be a factor of joint degeneration through excessive labral deformations [32]. In these studies, they particularly concentrate on simulation of hip motion in extreme position for dancers and athletes. However, the motions data are mostly attained from motion capture systems. Hence, the bones remain inaccessible and the estimation results are embedded with soft tissues such as muscles and fat.

In general, all studies mainly deliberate on calculation of $\mathrm{HJC}$ and simulation of rotational motions restricted by collision detection along with the selected anatomical axis of rotation [32] 
[36]. Predominantly a computer-assisted model would make simulation and analysis of motion much easier and palpable.

\subsubsection{Hip Joint Center}

Motion study regularly calls for determination of HJC location either by functional (dynamic) or predictive (static) approach. The functional approach estimates the HJC from recorded optical motion capture data that are recorded by using stereophotogrammetry or from computersimulation [28] [26] and the predictive approach estimates HJC based on relative position of anatomical landmarks.

The functional method was applied by calculating the centre of the best sphere described by the trajectory of markers placed on the thigh during several trials of hip rotations recorded by a motion capture system such as VICON [28]. The accuracy of the functional method depends on ROMs, the type and amplitude of the movement of the femur relative to the pelvis, markers location, and the number of data samples. Thus, it may be inefficient when motion is limited and it would result HJC location errors of $26 \mathrm{~mm}$ [26] [28] [37]. However, the error would be much more during motions like walking, ascending stair and descending stair [26]. While motion capture system is widely used for examining the functional HJC, this center can also be calculated through a computer simulated system. Kang et al. [6] reconstructed the 3D mesh surfaces from medical images. They set a temporary center of rotation for the hip based on geometry of the femoral head followed by simulation of rotational motions for the hip. Then, based on the fact that the center of rotation is fixed during the motion and also with keeping a constant joint space between the models, the center is displaced. The acquired center is the point 
of femur which remained fixed during circular movement of the hip. This functional method is experimentally accurate to within a tolerance of $\pm 0.1 \mathrm{~mm}$.

In contrast, prediction methods estimated the geometric HJC location based on distance between palpable anatomical landmarks by using regression equations and anthropometric measurements with the independent variables describing the geometry of the pelvis [38] [39]. HJC was predicted to lie directly distal to the midpoint of a line between the pubic symphysis iliac spine (PSIS) and the anterior superior iliac spine (ASIS) in a frontal plane projection and directly medial to the greater trochanter in the sagittal plane [39]. Generally, the location of HJC is expressed as a constant percentage distance between the markers either from skin landmarks [39] or directly from radiographs [38]. Calculated center may differ according to the location of anatomical landmarks and expert opinion [32]. This approach would result the HJC with an average error of 25-30 $\mathrm{mm}[28]$.

Moreover, Geometric HJC can be established by fitting a circle in 2D medical images or a sphere to the sphere shaped femoral head in 3D simulated model. Through computer-simulated markerless method, the center of femoral head can be calculated by using square fit method [33], Hough Transform method [40] or repetitively adjusting a sphere to convex part of the femur [41]

[6]. We introduce a method to estimate the geometric center of femoral head using Hough Transform method in the next chapter.

\section{Hough Transform Method}

The Hough Transform (HT) is a standard method for shape recognition in digital images [42] [43]. There are some broad advantages in this method including robustness to noise, shape distortions and occlusions/missing parts of an object. This method starts with passing the 
grayscale 2D images of object through an edge detection phase by utilizing Canny Edge detection method and the preliminary result would be a Boolean image. After that, the local gradient is determined for every point which is nonzero in the edge image. This gradient is employed to increment every point in the accumulator from a specified minimum to a specified maximum distance. Meanwhile, the positions of all of these nonzero pixels in the edge image are recorded. The centers are subsequently chosen as candidates from those points in the 2D accumulator if they meet the criteria to be above some known threshold and larger than all of their immediate neighbors. Based on the accumulator values of these candidate centers, centers are sorted in a descending order. As a result, the centers which have the most supporting pixels appear first. Then, for each center, all of the nonzero pixels which are built earlier are considered. These pixels are arranged based on their distance from the center. Resolving from the smallest distances to the maximum radius, a single radius is chosen that has the best support by the nonzero pixels. A center is accepted if it has adequate support from the nonzero pixels in the edge image and if it has an adequate distance from any formerly selected center.

\subsubsection{Fixed Center vs. Moving Center}

In some studies, it had been considered that the hip joint does not move around a fixed center of rotation, rather movement of the hip joint is associated with a firm amount of translation [44] [32] [41]. Menschik [45] determined that the both femoral head and acetabulum can be better represented as the conchoids shape rather than a perfect sphere (Figure 2.7). Therefore, as the result of conchoid morphology of the femur, there might be a translation for the center or rotation during the joint movements. 




Figure 2.7: The conchoids representation of the hip joint [45].

Puls et al. [27] tried to incorporate this knowledge into their application. They proposed an Equidistance method that used a dynamic hip joint center and attempt to maintain an equidistant joint space between the femur and acetabulum through additional femur translation during motion. The motions of cadaveric models were tracked by a navigation system and the locations of impingements were digitized with a pointer. Then, within the motion path, the impingement location and its extent were analyzed and evaluated [27]. On the other hand, Teschner et al. [25] mentioned that since the motion is restricted by impingement, the accuracy of HJC has no effect on the motion simulation result.

In this research, we examine the possibility of extreme ROM improvement based on bony morphology of the hip joint; explicitly by moving the HJC through a digital simulation. Similarly, it has been claimed in a recent research by Yazdifar et al. [36], who were able to demonstrate that changing the center of rotation inward and outward the acetabulum changes the exact location of impingement and subsequently the risk of FAI. They performed an experiment on a patient diagnosed with Cam FAI. The CT scans of the patient hip joint were acquired and the motion data were obtained through a motion capture system. Corresponding to their 
observation with displacement of HJC outward the acetabular, there would be a significant rise in the ROM and will change the angle at which the impingement happens.

However, both spheres and conchoids provided a good approximation of hip joint geometry and the difference in estimation of HJC derived from conchoids or sphere is not substantial (fitting error less than $0.5 \mathrm{~mm}$ ) [45]. As the simulation of motion for conchoids shape is nondeterministic, hence most of the researches in the area of motion simulation and impingement detection of the joint come with sphere representation of the joint.

\subsubsection{Motion Capturing and Simulation}

The hip has an essential role in most activities of daily living. Not only is the hip responsible for "distributing weight between the appendicular and axial skeletons, also it is the joint from which motion is initiated and executed" [7]. Several studies have shown that hip motion is restricted in the joint with morphological abnormalities and correspondingly the ROM is impaired. Moreover, the acetabular cartilage is at the risk of being attenuated as the result of abnormal shear stresses [41] [46].

On the other hand, Extreme motions may lead to early OA and inversely the abnormality of hip may cause reduction in extreme motion. Kennedy et al. [30] attempted to estimate and assess the effect of Cam FAI on the maximal dynamic motion of hip by using a motion capturing system. Their results revealed that maximal hip dynamic mobility decreased in internal rotation and abduction, flexed external rotation, total hip transverse ROM and total hip sagittal ROM in the FAI group compared with the healthy matched control.

Furthermore, some researches indicated that some dancing movements may damage the hip joint, which lead to early osteoarthritis specially FAI. In a recent research, the captured motions 
of dancers were simulated and impingement was detected using computer assisted technology [4]. They demonstrated that the frequency of impingement and amount of subluxation increased with actuating significant stress in the dancers' hip joint during extreme motions.

Generally, a digital simulation of hip joint provides better understanding of motion and pathology of the hip joint. Computer simulation of the hip joint usually starts by reconstructing 3D meshes from cadaveric, CT [47] or MR Images and estimating the HJC [48] [28]. Once the 3D model is constructed, the desired medical rotation can be interactively applied on the joint by rotating the virtual femur about estimated HJC [48].

Some software is also available to simulate and analyze the hip motion. Charbonnier et al. [43] presented a software which is designed to improve hip joint $\mathrm{OA}$ understanding using 3D anatomical models that are constructed from MRI and based on motion capture data. The software constructs 3D hip models from MRI data and based on the optical motion capture data, system computes the hip joint kinematics and ROM. Moreover, a collision detection algorithm is used to virtually locate abnormal contacts between the proximal femur and the labrum.

However, the kinematics data including the HJC and ROM values are mostly obtaining through a motion capture system, even in the current computer simulation systems. A motion study based on markers and motion capture systems have disadvantages of being dependent and sensitive to the markers placement, skin cloth artifacts and generic calculation. In addition, it requires a specialized experimental room setting for motion capture and it is difficult and painful to obtain maximal motions. In addition, it is difficult to isolate the effect of bone outlines out of anatomical factors such as muscles, tendon and other connective tissues which are merged in 
motion capture in real-life. Thereby a versatile markerless simulation of hip joint kinematics and ROM improves accuracy and efficiency of the system.

Despite the studies on simulating hip kinematics and analyzing the effect of different kind of FAI on the ROM [6] [49] [5] [41], there is no much research in the area of $3 \mathrm{D}$ visualization of hip ROM envelope from 3D segmented MRI model. Hence, we are inspired to develop a simulation and visualization tool to estimate the maximum impingement-free ROM from bony structure of the hip to evaluate the maximum possible mobility of the joint without considering the soft tissue effects. The $3 \mathrm{D}$ visualization system can also help to improve communication between surgeon and patients.

\subsubsection{Computer Simulation of Impingement Detection}

Impingement detection (also known as interference detection) is performed by using a collision detection method in the computer simulation. It aims to automatically report a geometric contact when it is about to occur or has actually occurred. For many years, collision detection has been one of the most interesting topics in the field of computer graphics, computational geometry, robotics and especially medical simulation. Furthermore, collision detection is considered to have an important role in motion analysis and simulation in the kinematics and kinetics evaluation of human joints, particularly hip joint.

In many of these application areas, collision detection is considered as a major computational bottleneck. In the recent years, tremendous numbers of approaches have been proposed in order to tackle the collision problem for rigid and deformable objects. In general, the geometric models can be classified as polygonal objects, splines or algebraic surfaces. All the existing approaches involve contact analysis and spatial reasoning in static and dynamic environments. In the past 
surveys [50] [51], the existing collision detection methods are mainly classified into bounding volume hierarchies (BVH) [52] [51], distance fields [53] [51] [54], ray tracing [55], image-space techniques [56] [50] [51], spatial subdivision [53] and stochastic method [25] [57].

The following section 2.4 focuses on the general collision detection approaches which can be used in any applications for computerized objects. The applications on the hip joint area are described in section 2.4.6.

\subsection{Collision Detection Methods}

This section describes in details the current existing collision detection methods which are mentioned in section 2.3.3. Medical adaptation to this particular area of hip impingement is described in the last subsection while other sections describe more the general methodologies of collision detection.

\subsubsection{Bounding Volume Hierarchy}

Bounding volume hierarchies (BVH) are the most common data structures used for collision detection either for rigid or deformable objects [55] [51]. BVH recursively and iteratively divides the object into different partitions. BVH can be called as "discrete representation of level of details of objects" [50]. BVH prunes and checks the collision from first level to the leaf level with the topology of the tree. Hierarchy includes a coarse representation of the object at first

level and includes more details of the object at further levels. At the last level which is the leaf level, the hierarchy generally includes the object primitives (lines, triangles). Various types of bounding volumes are used, such as axis aligned bounding box (AABB) trees [58] [52], oriented 
bounding box (OBB) trees [59], discrete orientation polytopes (DOPs) [60] [61] and convex hull trees [62].

In AABB data structure, all the boxes in a tree have the same orientation since the boxes are aligned to the axes of the model's local coordinate system [58]. AABBs in the trees are then transformed as the models are moved or rotated in the scene [52]. On the contrary, OBBs can be arbitrarily oriented respect to coordinate axes and yield to approximate objects tighter than AABBs but updating an $\mathrm{OBB}$ tree is significantly more complex. Although AABBs can be implemented much easier [59], but if two objects are very close and have multiple contacts, the performance slow down and become a major bottleneck in the simulation. Meanwhile, Discrete orientation polytopesa (k-DOPs) was proposed as a bounding volumes to provides more precise tightness compared to bounding spheres and AABBs and relatively lower cost of interference tests and updates compared to OBBs and convex hulls [60]. k-DOPs are convex polytopes whose facets are determined by half spaces with outward normals coming from a small set of $\mathrm{k}$ orientations [60] [61]. However, the surface distance information and depth penetration is neglected in all of the BVHs variety.

In general, the real-time calculation of updating BVHs is difficult to be achieved and it involves coordinate transformation and realignment [50]. Moreover, BVHs are inefficient at distance computation and it is not preferred for computationally expensive simulations. In addition, the computations performed in these methods are not efficient enough for joint-related applications, due to their generality [35]. 


\subsubsection{Distance Field}

Distance field specifies the minimum distance to a closed surface for all points in the field and it will separate the space into inside space and outside space [51] [63]. Distance field is sometimes called distance volumes [64] or distance functions [65]. The distance map of the object surface is computed by a specific distance function and the collisions queries are performed on these values. In addition, the evaluation of distances and normals needed for collision detection and response is extremely fast and independent of the geometric complexity of the object.

Uniform 3D grids, octrees and BSP-trees are different data structures representing distance fields [63]. Uniform grids computes distance values for each grid point and reconstruct intermediate values by trilinear interpolation. These data structures are easy to implement and computation time is short. On the other hand, uniform grids needs huge memory specially when representing a high resolution object with sharp features. So as to tackle these problems, adaptively sampled distance fields (ADFs) was proposed by Frisken et al. [66] which stores data in a hierarchy. This feature increases the sampling rate in regions of fine details. ADFs are usually stored in an Octree (one node has up to eight child nodes) among various spatial data structures. During construction of ADFs, each cell subdivides until it reaches maximum tree depth. ADFs provide a good compression ratio as stated in [51]. For collision detection purposes, special care has to be taken in order to guarantee continuity between different levels of the tree Compared to uniform grids.

BSP-tree uses a piecewise linear approximation [67] of the distance field, which is not necessarily continuous. Memory consumption can be reduced by using BSP-tree data structure 
even further [67] but unfortunately, the construction of the BSP-tree is computationally expensive [67]. Finding the minimum distance between a given point and a triangle can be done efficiently using Voronoi regions of the features of the triangle [56]. A discrete Voronoi diagram [56] is computed by rendering a three dimensional distance mesh for each Voronoi site (a set of primitives). A Voronoi diagram divides space into regions, where each region consists of all points that are closer to one site than to any other. This algorithm has to proceed slice by slice due to complexity of 3D distance meshes. Thus, a huge number of triangles have to be rendered, slowing down this method considerably.

Distance fields provide highly robust collision detection, since they divide space strictly into inside and outside but it requires a time consuming preprocessing step. However, distance fields computation has to be continuously updated like BVHs and real-time implementation of deformable collision detection using this method is difficult.

\subsubsection{Spatial Subdivision}

Spatial subdivision is a simple and versatile method which speeds up the collision detection process by utilizing discrete cells [53] [51]. Different types of grids are used including uniform grid, spatial hashing, octrees, kd-tree and BSP trees. In general, algorithms based on spatial subdivision are independent of topology changes of the object [51], which makes it particularly interesting for deformable and rigid objects. Moreover, spatial subdivision can be used to detect collision and self-collision. Algorithm divides the space into certain cells and it checks collision for each grid cell. Consecutively, it tests all the primitives inside the collide grids. Collision detection based on spatial subdivision depends on some parameters such as the size and the shape of the grid cells [54]. Experiments suggest that the optimal cell size is about the same as 
that of the bounding box for a single object primitive. In spatial subdivision the data structure must be chosen very precisely to be efficient and it is flexible with respect to computational time and memory [53].

\subsubsection{Image Space Base Technique}

Image space base techniques are usually convenient to implement on the graphics hardware (GPU) [50]. This technique usually utilizes projections of objects in order to accelerate queries computations. Since they do not require any time-consuming preprocessing or special data structure, they are especially appropriate for environments with dynamically deforming objects [51]. This method can be used to detect collisions and self-collisions like spatial subdivision method. It uses frame buffer operations to implement a virtual ray casting algorithm for every pixel that detects interference between objects [68]. The edges of the objects are written to the depth buffer and the objects which they penetrated each other are detected by using a virtual raycasting algorithm [68]. Since image-space techniques work with discretized representations of objects, they do not provide exact collision information [50].

\subsubsection{Stochastic Approach}

Recently, inexact methods have become a point of interest in collision detection researches. A stochastic approach [51] detects collisions on the basis of statistical information and probabilistic principles. Stochastic methods select random pairs of colliding features (vertices, edges, triangles, etc.) as an initial guess of the potential overlapping regions. Hence, this technique trades off accuracy for computation time. In this method, approximated polygonal models are established. Whenever an object deforms or moves the overlapping region at a time, still remains 
as interest region in the next time. This method is restricted to rigid objects. However, accurate collision detection cannot be achieved since it is based on the probability. Therefore, a realistic simulation is impossible to be accomplished with stochastic methods. At the same time, "humans cannot distinguish between physically-correct and physically-plausible behavior of objects" [57]. Therefore, it is accepted to enhance the performance of collision detection, while its precision is reducing.

\subsubsection{Collision Detection for Hip Joint}

Some collision detection methods are proposed to be efficient enough in medical simulation. In this section, we discuss about some methods which are highly adapted in biomechanical simulation of hip joint where the meshes are quasi-spherical [34] or near-spherical [33]. Arbabi et al. [35] proposed a collision detection method which relies on cylindrical segmentation of space around a given rotation axis. During cylindrical segmentation of space both vertices of fixed and mobile objects are mapped from Cartesian coordinates to cylindrical coordinates. Therefore, the space is then cylindrically partitioned into ring cells. Each fixed object surface polygon indices in corresponding cells of intersecting ring. Then, the algorithm searches for collision for the ring cell containing each mobile vertex. It is assumed that the mobile object is rotating around constant rotational axes. Hence a mobile vertex can only collide with the fixed object surface polygons stored in the unique associated ring. The colliding pairs and penetration depth are computed by checking the angular distance between the mobile vertex and the fixed object surface polygons indexed in the table cell in the direction defined by the rotation axis. Although this method provides the penetration depth details, sometimes two surfaces are close enough to create forces on the surfaces without any penetration. Therefore, this method does not 
have flexibility to provide strain or stress information. Moreover, it has a long computational step since each vertex of mobile object is cylindrically projected to fixed object surface for every rotational axis and also penetration depth is estimated for all rotational angle of individual vertex. Relying on theses characteristic, the cylindrical segmentation method won't be a good choice for complex objects.

Maciel et al. [34] recommended a spherical sliding method which proceeds in two stages. During initialization step, a mesh under a certain conditions is defined as reference mesh (fixed mesh) and it is spherically sampled. The proximities information of two meshes is stored in cells of a table named Spherical Sliding Table (SST). Each table cell uniquely characterizes an orientation of a ray from origin of reference mesh toward second mesh to determine the closest intersect face. This is achieved by exhaustively searching for each vertex of a mesh model A; as reference mesh, is near to which triangle in model B. During collision detection step, this SS table is exploited as for every vertex of second mesh (mobile mesh); its signed distance to the closest SST of reference mesh is obtained and compared. The approximation resulting from the sampling allows this approach to be exploited for real-time interactions. However, the method returns approximated collision results which are not suitable for accurate applications.

Similarly, Cai et al. [33] proposed an impingement detection system named Rapid Spherical Impingement Detection system (RSID) based on sampling algorithm and surface-to-surface distance measurement in order to observe the impingement between acetabulum and femur. In the sampling step, the hip joint is considered as a ball and socket joint, so the geometric coordinates of triangulate mesh models are mapped to two dimensional (2D) spherical coordinate grids with the distance data from the origin. For each triangle of model, the sample points are generated through polygon rasterization and their distance data are stored in a data structure 
named look-up table (LUT). The distances of densely sampled points of the mesh surfaces are interpolated from triangle vertices. Then, the impingements are detected based on the distance difference of acquired sample points when one object is on or inside the other object surface.

Collision detection approach proposed by Cai et al. [33] has some similarities to the method proposed by Maciel et al. [34], since both methods are based on spherical sampling and surface distance evaluation. However, RSID system is based on more efficient and accurate sampling method and data structures. Sampling rates in RSID is set as $7200 * 3601$ (precision of $0.05^{\circ}$ ) in real-time, conversely Maciel et al. [34] varied the size of the SST from $10 * 10$ to $400 * 400$ (precision of $0.45^{\circ}$ ) which is much less resolution. Moreover, evaluating the proximity of one vertex to one triangle in an orientation is not a good strategy since more triangles may appear in same orientation. The inefficiency and inaccuracy of Maciel's method lead poor model geometry information in addition to a high computation cost $\left(\mathrm{O}\left(\mathrm{n}^{2}\right)\right)$. RSID is a rapid collision detection system which is efficient for joint simulation and also is applicable to deformable objects. Moreover, it is fast enough for real-time simulations at millisecond level of computing with less computational cost $(O(n))$. Therefore, we implemented our collision detection algorithm based on this method. 


\section{Chapter 3. Overview of Methodology}

In this chapter, we show how our system is designed and implemented to simulate and visualize the maximum motion of the hip joint which is achievable from bony morphologies of the femur and the acetabulum as illustrated in Figure 3.1.

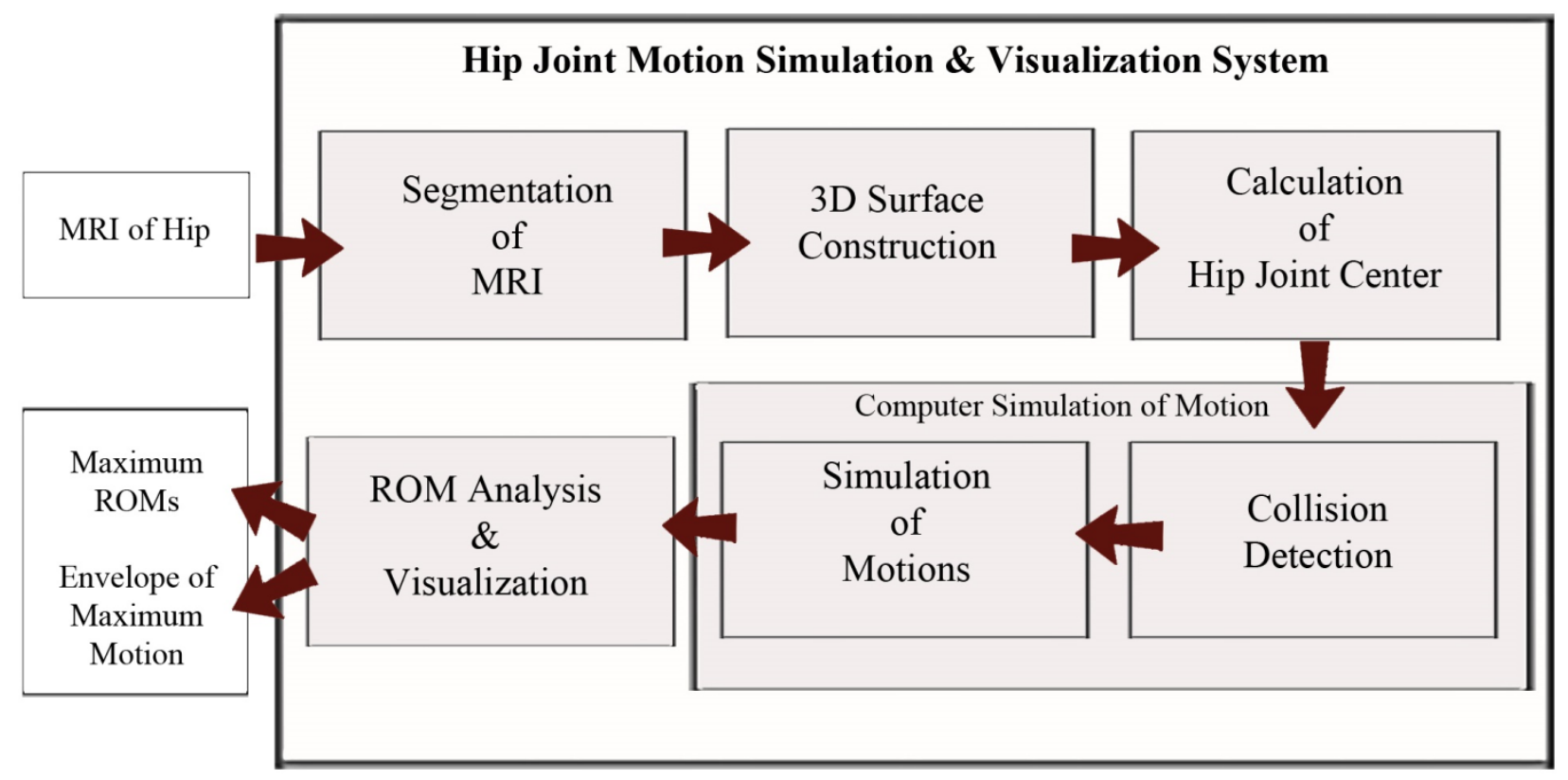

Figure 3.1: High-level view of our motion simulation and visualization system.

Our motion simulation and visualization system starts with segmentation of bones from MRI and construction of 3D bone models, followed by motion simulation around the calculated HJC with the control of collision detection algorithm. The motion simulation aims to compute the maximum ROMs of the joint in each direction, as well as visualization of maximum motions envelopes. 
As described in the literature review, motion simulation and analysis call for modeling of the hip joint. The HJC is estimated over the 3D constructed anatomical surface models which are segmented from medical images dataset; MRI data is used for our experiment, since it has advantage of not using radiation while $\mathrm{CT}$ scans do. Moreover, MRI is more versatile than the $\mathrm{X}$ Ray and is used to examine a large variety of medical conditions and also depicts both soft tissue and bone. The surface models of bony hip joint after 3D construction are enhanced and refined to ensure the accuracy and efficiency of the system.

The subsequent articular surfaces of the hip joint, femoral head and acetabulum, are assumed to have spherical shapes as the ball-and socket joint where the femoral head is considered as the ball while the acetabulum as the socket. We start with assumption of sharing a common center between the acetabulum and the $\mathrm{HJC}$ that is the center of rotation of the femoral head. Then, joint motion is designed around the fixed $\mathrm{HJC}$ as constrained by impingement avoidance algorithm depending on bony anatomical of the joint. The femur rotates around the estimated HJC and stops whenever impingement is detected using a collision detection algorithm. ROM is calculated as the degree of maximum possible impingement-free motion in every direction from bony outline of the joint without considering the surrounding connective soft tissues factors. Later on, we break the assumption of the common center in section 3.4.2. Hence, the center of acetabulum is calculated in addition to center of femoral head (HJC) and the effect of translation of the HJC in direction of acetabulum center on the maximum ROM is evaluated in section 4.3.

\subsection{Construction of 3D Hip Bones}

Human joint computer simulations usually start with constructing three-dimensional (3D) meshes of the joint tissues (bones, cartilages, etc.) from CT or MR Images [47]. MR images can 
be acquired at an arbitrary orientation to optimally attain the 3D structure, while CT would call for reformation of axial data, typically resulting loss of resolution. CT datasets can be good candidates to make 3D bone surface models, but due to high resolution of these images and high exposure to radiation, it is risky to the patient [69]. On the contrary, MRI datasets are good materials for making 3D surface models and they are proper for the purpose of our study.

On the other hand, majority of femur and acetabulum meshes which are available online by various websites and companies suffer from lack of scientific structure and topology. Moreover, there might be collision between the femur and the acetabulum in the neutral pose of these models. Therefore, they are not suitable to be considered as the system inputs. In this section we explain the process involved in our system to construct 3D bones of the hip joint from MRI.

\subsubsection{Segmentation of MRI}

In recent years, the number of software to segment 3D medical images and construct 3D data has been growing rapidly. To achieve highly accurate 3D models of the patient's anatomy, ITKSNAP and Mimics are the most commonly used toolkits which are freely available. In this study, ITK-SNAP 2.4.0 software is used to construct the 3D bone of the hip from MR images of a male patient. It utilizes active contour techniques leading a semi-automatic segmentation .It provides manual delineation and image navigation to its users. One hundred and sixty MRI slices in format of Digital Imaging and Communications in Medicine (DICOM) files are imported into the software and segmented semi-manually (Figure 3.2) to obtain 3D point clouds of the femur and the pelvis models (consist of 51245 and 126402 vertices respectively) (Figure 3.4 (a)). After that, the segmented results are verified by a medical doctor from Ottawa General Hospital. 

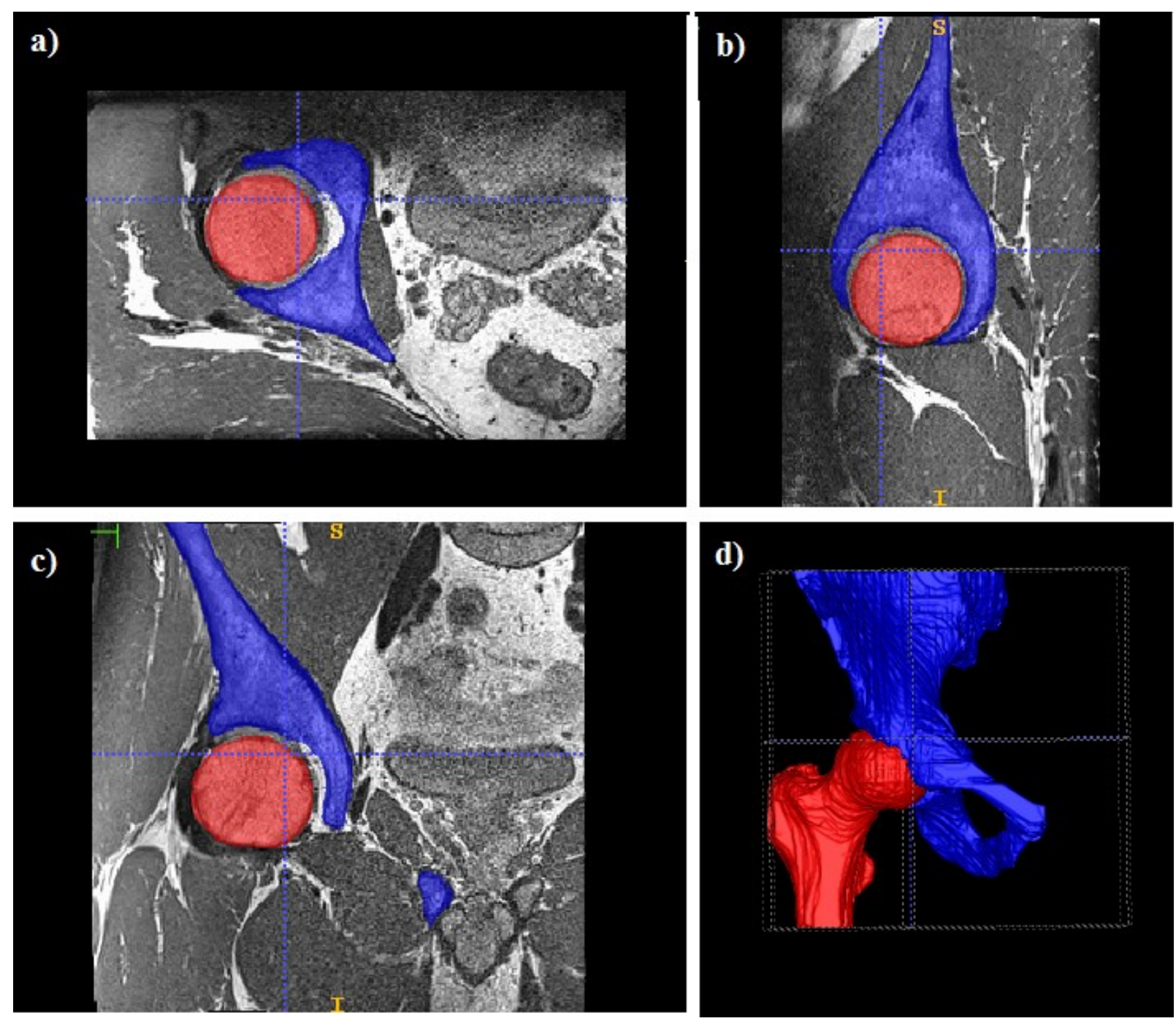

Figure 3.2: a-c) segmented MR images in axial, sagittal and coronal planes from ITK-SNAP. d) $3 D$ constructed femur (shown in red) and pelvis (shown in blue) models.

As we mentioned in section 2.1.1, bones consist of two distinct morphological type of bones; compact and spongy bones. The spongy bone is surrounded by a compact bone shell which is very thin and its thickness varies between 1-5 mm. Compact bone shell is thicker at the body (shaft) of femur than its head (Figure 3.3). However, compact bone is easier to distinguish according to its high contrast compare to surrounding soft tissue. Consequently, the femoral head 
is harder to segment than femur body due to its proximity to the acetabulum and containing much thinner compact bone. In our study, the compact bone is segmented too. Therefore, the constructed 3D models of the bones as shown in Figure 3.2(c) are made of both cortical and spongy layers of bones.

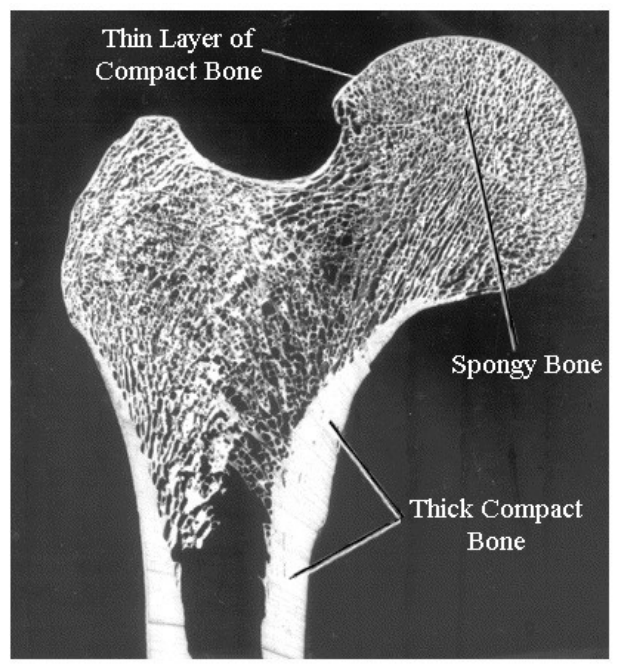

Figure 3.3: Cross-section of upper femur that displays compact and spongy bones distributions [70].

\subsubsection{Construction of 3D Surface Models}

A surface construction algorithm called the Ball-Pivoting [71] is applied to incrementally build an interpolating triangulation of the acquired point clouds. This technique is efficient in terms of execution time and storage requirements and robust enough to handle the noise present in real data. Meanwhile constructed polygon meshes of femur and acetabulum may contain degenerate elements, self-intersecting or overlapping parts, surfaces holes, and many other flaws that make them not suitable for a widespread usage. Consecutively, an automatic repairing procedure proposed by Attene [72] is used to remove all the aforementioned defects and 
transform the raw digitized meshes into single manifold and watertight triangle meshes (Figure 3.4(b)). The complexity of obtained triangulated meshes has been reduced to 7943 and 37536 vertices for the femur and the pelvis respectively by Autodesk Meshmixer08 software with the purpose of lower computation and less memory cost (Figure 3.4(c)).

The main idea of the construction of computer-assisted model is that $3 \mathrm{D}$ anatomical models can provide valuable insight into the understanding of hip pathology and motion visualization. After construction of 3D mesh surfaces, some calculations are carried out and further motion analyses are performed on these gained models.

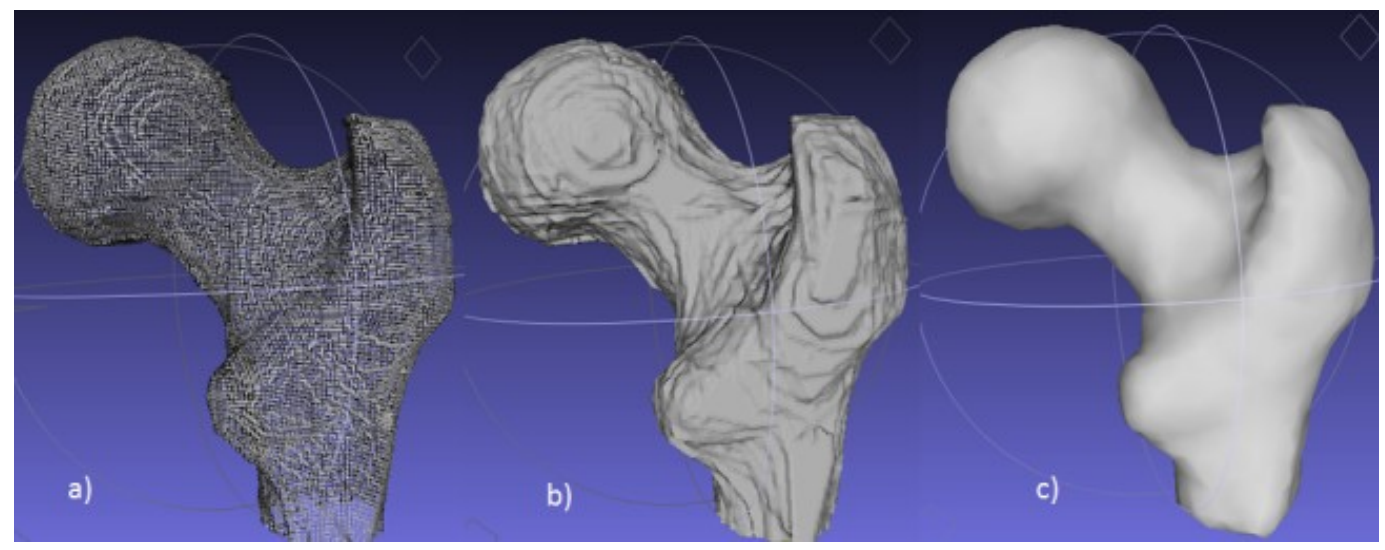

Figure 3.4: a) Point cloud constructed from segmented MRI of right femur. b) Result of 3D mesh reconstruction and geometry correction proposed by Attene [72] c) 3D model surface after reducing its complexity.

\subsection{Hip Joint Center Calculation}

Hip joint exhibits a ball and socket joint; hence at this stage of research, the center of hip joint is considered as the center of ball which is femoral head. Geometric calculation of the HJC exploits estimation of best sphere fitted to the sphere portion of femoral head. This center 
depends on the morphology of bony outline of the femoral head. Accordingly, the geometric femoral head center is computed in three main steps as follow:

(i) 3D to 2D projection: 3D femur mesh model constructed from MRI is converted to $2 \mathrm{D}$ by orthogonal projection in four different views (left/right lateral, anterior and posterior). For each anatomical view of femur, the system captures a 2D image in gray-scale.

(ii) Image Processing: For each view, Gaussian filter has been applied to 2D gray scale image in order to remove noises and then Hough Transform method (described in section 2.3.1) is performed in smoothen images to estimate the center and radius of circle fitted to the femoral head in 2D as shown in Figure 3.5.

(iii) $2 \mathrm{D}$ to $3 \mathrm{D}$ projection: In the last step, the $2 \mathrm{D}$ images are projected back to $3 \mathrm{D}$ and an average center from different views is measured.

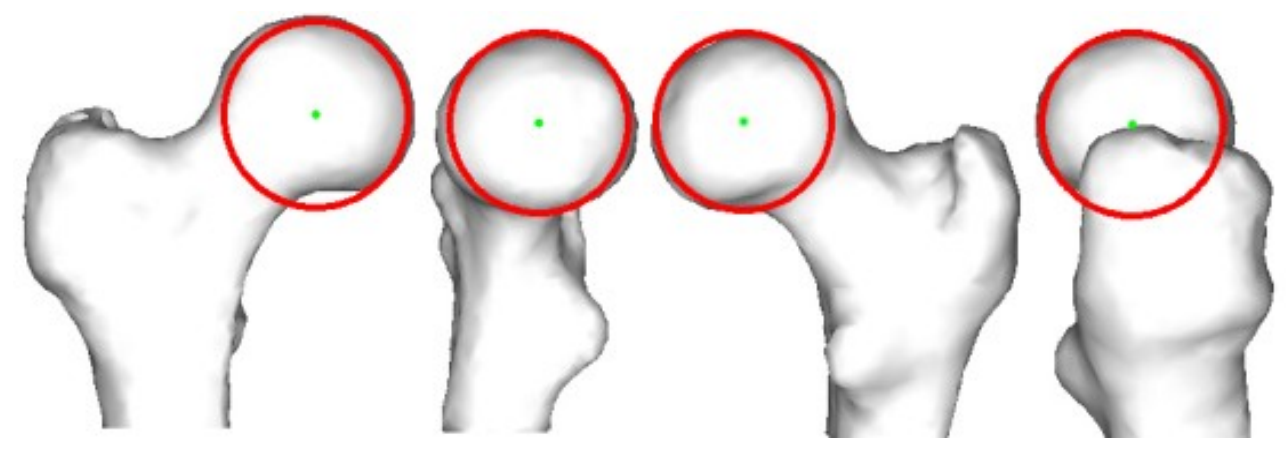

Figure 3.5: Result of Hough transform in anterior, right lateral, posterior and left lateral views of a right femur model (respectively from left to right). 


\subsection{Collision Detection for Ball-and-Socket Joint}

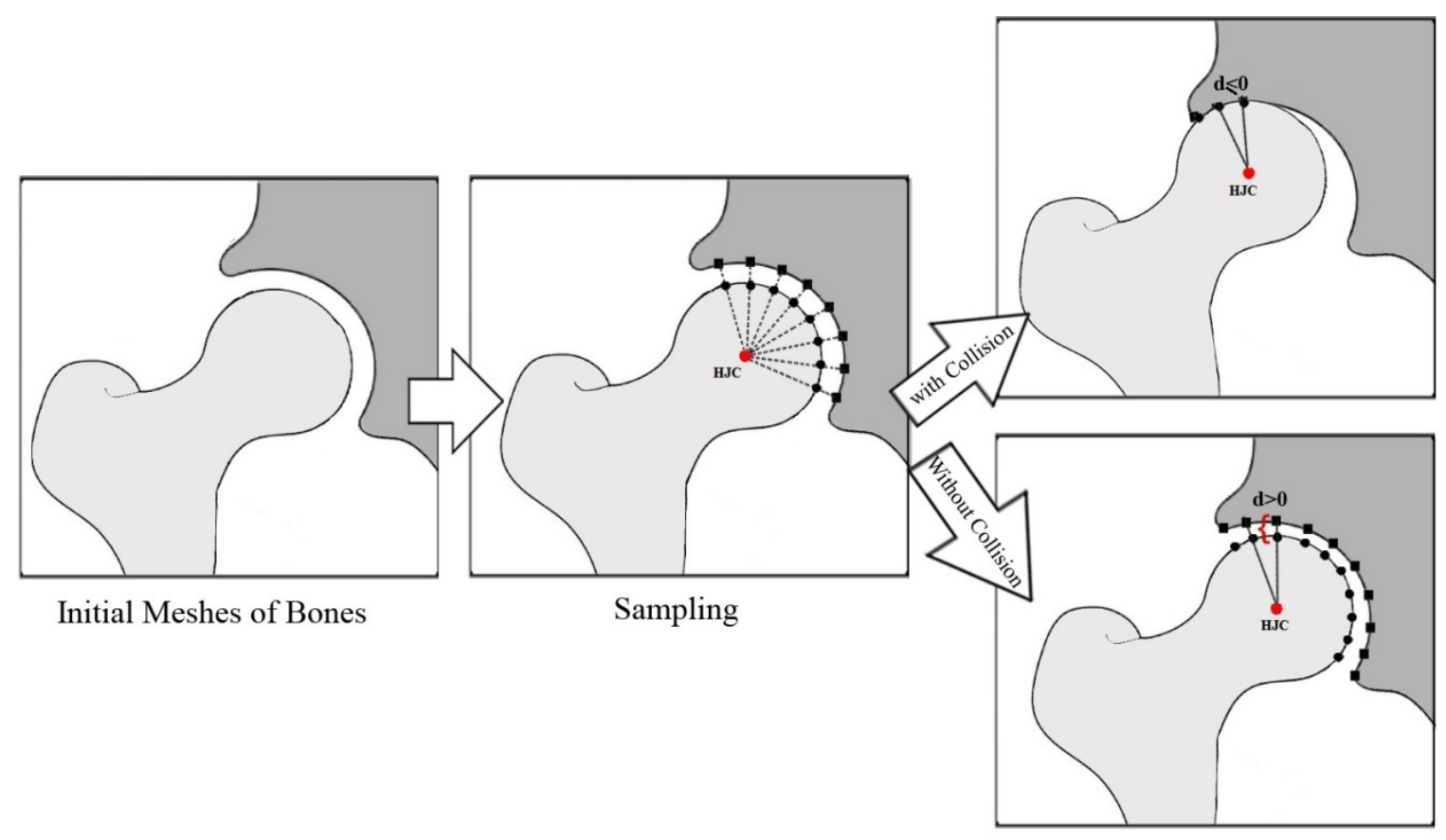

Collision Detection

Figure 3.6:2D sketch of our collision detection algorithm for ball-and socket joint from left to right: The surfaces of the hip bones are sampled according to HJC (red dot) and checked for collision. If the distance (d) between samples of two surfaces is less than or equal zero, there is collision between two surfaces otherwise no collision is detected.

Simulation of hip motion requires precise collision detection [6] [25] when 3D computerized model is constructed from segmented CT, MRI or etc. While tremendous number of collision detection techniques for rigid bodies has been recommended in static and dynamic environment, we need a highly efficient collision detection algorithm to detect the real time interaction between the femur and the acetabulum during motion. Therefore, inspired by Cai et al. [33] and Maciel [34], we implement a collision detection system as shown in Figure 3.6 to realize realtime interaction between the proximal femur and the acetabulum during hip motion. The system 
is developed as it detects all type of impingements covering intra-articular, anterosuperior and poster-inferior impingements. Detection of collision between two 3D surface models is performed in two main stages. First at the sampling phase, the quasi-spherical portions of the femoral head and the acetabulum surface models are spherically sampled. Then, at collision detection phase, based on distance difference between two sampled surface models, collision has been detected.

\subsubsection{Sampling Phase}

At the sampling phase, the system gets the triangulated proximal femur and pelvis models and extracts their quasi-spherical portion based on fitting sphere to the femoral head and the acetabulum rim independently. Sphere fitting to the femoral head is described in the aforementioned section (3.2). For the pelvis model, the sphere fitted to concave acetabulum is estimated based on placement of control points (markers) on the acetabulum where for our experiment four control points is used (Figure 3.7).

From analytic geometry, we know that there is a unique sphere that passes through four noncoplanar points if, and only if, none three combinations of four points be collinear. So four control points $\left(x_{1}, y_{1}, z_{1}\right),\left(x_{2}, y_{2}, z_{2}\right),\left(x_{3}, y_{3}, z_{3}\right),\left(x_{4}, y_{4}, z_{4}\right)$ are set up on the acetabulum, three points on the acetabulum rim and one on its depth as shown in Figure 3.7(a). The sphere with center $(X, Y, Z)$ and radius $(R)$ is evaluated based on the polynomial equation (1) and it is acquired by solving determinant equation (2).

$$
\left(x_{i}-X\right)^{2}+\left(y_{i}-Y\right)^{2}+\left(z_{i}-Z\right)^{2}=R^{2}, i=1,2,3,4
$$




$$
\left|\begin{array}{lllcc}
x^{2}+y^{2}+z^{2} & x & y & z & 1 \\
x_{1}^{2}+y_{1}^{2}+z_{1}^{2} & x_{1} & y_{1} & z 1 & 1 \\
x_{2}^{2}+y 2^{2}+z 2^{2} & x_{2} & y_{2} & z 2 & 1 \\
x_{3}^{2}+y 3^{2}+z 3^{2} & x_{3} & y_{3} & z 3 & 1 \\
x^{2}+y_{4}^{2}+z 4^{2} & x_{4} & y_{4} & z 4 & 1
\end{array}\right|=0
$$
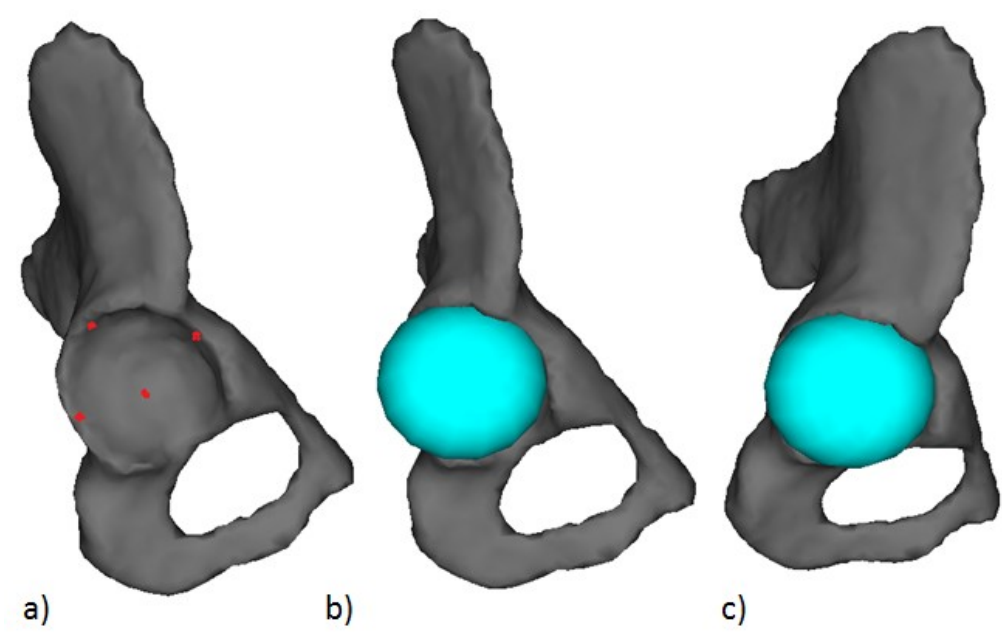

c)

Figure 3.7: Calculation of acetabulum center: a) four markers (red dots) on acetabulum is set up, three on the rim and one on its depth. b,c) Result of spherical approximation of acetabulum.

For each of the proximal femur and the acetabulum models, one bounding sphere is placed in their local coordinates which is centered in the center of its tightly fitted spheres with the radius, $R^{\prime}$. Radius $R^{\prime}$ is set to be greater than $R$ to ensure the efficiency of the system in detection of anterosuperior and poster-inferior impingement (Figure 3.8). Consecutively, those triangles which are sited inside the bounding spheres are selected and listed to be sampled. The geometrical information of these vertices is stored in a table named Sampling List (SL). 
Moreover, if a triangle vertex or edge is placed inside the bounding sphere, it is taken into consideration and is added to the SL tables to improve accuracy of system.



Figure 3.8: Illustration of bounding sphere (red color) for the acetabulum that is localized on the center of acetabulum with radius $R^{\prime}$ ( $R$ is the radius of the sphere fitted to quasi-spherical portion of the acetabulum).

Generally, digital simulation of collision detection is according to bones surfaces collision. Therefore, the surfaces of 3D bone models; femur and acetabulum are sampled and more points in the sampling mean more accurate collision detection between two surfaces. However, the computation burden is higher.

It is noted that the geometrical information of the acetabulum is calculated according to the HJC (center of the femoral head) which is also center of world.

In order to sample the surface of two triangulated mesh models, two sampling methods are implemented and compared in the following subsections. First, sampling method is based on random point generation inside each triangle stored in SL tables. This sampling method is carried in 3D Cartesian coordinates. On the other hand, another method is developed which is sampling 
based on rasterization. The triangles which are stored in SL table are mapped to 2D spherical coordinates and consecutively are sampled based on rasterization and interpolation with coefficients of barycentric coordinates. The sampled points of each model are stored for further analysis in a data structure called LUT which is a 2D array that is indexing by the spherical coordinates. However, in our collision detection algorithm, the surfaces of listed triangles in SL tables are sampled as the method described in section 3.3.1.2 to ascertain the collision between the models during hip motion.

\subsubsection{Random Generation of Sample Points}

One way to sample the surface of a mesh model is to generate a number of random points within each triangle bounds until we get a desired number of samples as shown in Figure 3.10. This method is an easy method to sample a space or surface with variable density. In order to guarantee a more uniform coverage of the samples, it's better to weight the probability for a triangle to be sampled according to its area, so that larger triangles will be sampled more often and hold more samples along their surfaces. Therefore, our method for generating random sample points inside each triangle proceeds as follows:

First, we iteratively go through all triangles of triangulated mesh models (femoral head and acetabulum) which are stored in SL table and for each triangle; we compute its area along with the cumulative area of triangles visited so far. The total number of sample points for $\mathrm{K}$ triangles is set to $\mathrm{N}=1024^{2} 1024^{2}$ for the initial experiment. Then, we pick the number of sample point $n_{i}$ for each triangle with probability proportional to its area. The area of a triangle with vertices $\mathrm{A}, \mathrm{B}$ and $\mathrm{C}$ is half of the magnitude of the cross product of vectors $\mathrm{AB}$ and $\mathrm{BC}$ as equation (3): 


$$
\text { area }=\frac{1}{2} *|A B \times B C|
$$

The sample point $P$ inside the triangle $T$ with the barycentric coordinates, $u$ and $v$, is obtained by generating two random number, $\xi_{a}, \xi_{b}$, between 0 and 1 as following equations (4) (5).

$$
\begin{aligned}
& u=1-\sqrt{\xi_{a}} ; v=\xi_{b} * \sqrt{\xi_{a}} \\
& P=u * A+v * B+(1-u-v) * C
\end{aligned}
$$

Where $u \in[0,1], v \in[0,1]$ and $u+v \in[0,1]$.

Intuitively, $\sqrt{\xi_{a}}$ sets the percentage from vertex $A$ to the opposing edge, while $\xi_{b}$ represents the percentage along that edge (Figure 3.9). Taking the square root of $\sqrt{\xi_{a}}$ gives a more uniform random point with respect to surface area.

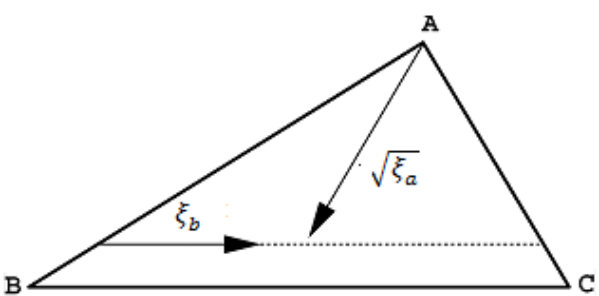

Figure 3.9: Sample a random point in triangle


Figure 3.10: Random generation of sample points on femoral head surface proportionate to each triangle area of mesh. 
Similarly, Sik et al. [73] proposed a sampling approach based on predefined density for hairs modeling applicable in computer animation. However, this sampling method is not efficient enough for our collision detection system, since it speeds down the processing time of detection phase as it is random and nondeterministic. Slow convergence and large open spaces between samples are other disadvantages of this methodology. Although, the large open spaces can be reduced by increasing the number of sample points, this requires vast computation and storage leading to decline the speed of computation. Therefore, in the following section 3.3.1.2, we introduce our sampling method which is not only more robust, but also more efficient in term of computational time.

\subsubsection{Spherical Sampling based on Rasterization}

We develop our sampling method inspired by the proposed algorithm by Cai et al. [33] based on rasterization with the resolution degraded to 0.05 per unit (sampling precision) as shown in Figure 3.11. Rasterization is a process in computer graphics and image processing which transforms the geometric primitives (triangles, circles, polygons) into the pixelised images (raster image) and is achieved by sampling the scene at the pixel intervals. With smaller pixel size, image has higher resolutions. 

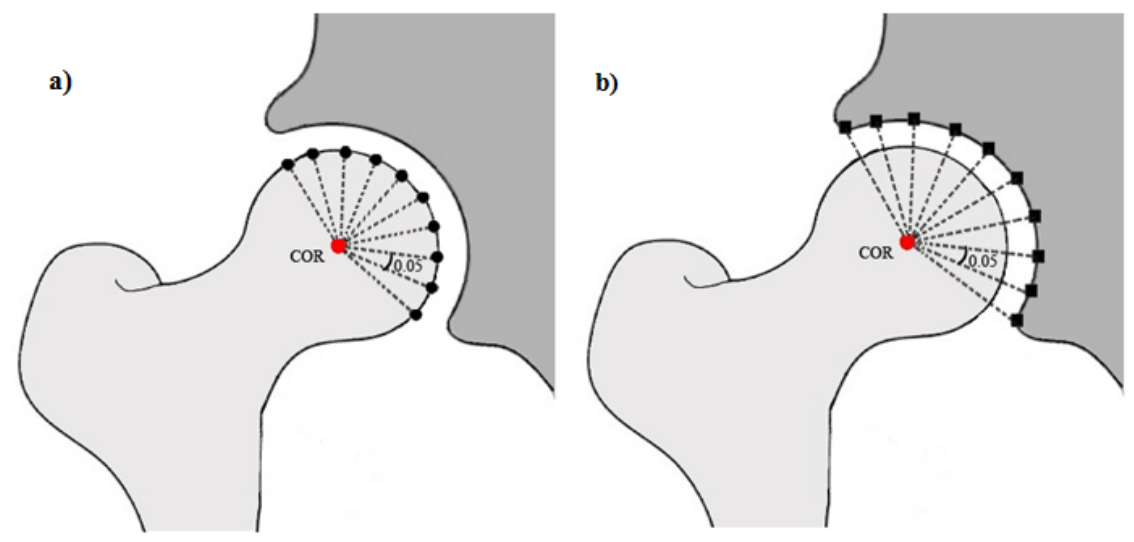

Figure 3.11:2D conceptual illustration of surface sampling (sampling precision of $0.05^{\circ}$ ): red dots represent HJC as center of spherical coordinate. Dash lines demonstrate the sample points distance from the center. (a) Black dots represent instances of sampling points on the femoral head surface. (b) Black squares represent the generated sample points on surface of acetabulum.

At first, we map the models from $3 \mathrm{D}$ coordinate to $2 \mathrm{D}$ grid. The accumulated triangles in SL tables of both mesh models (proximal femur and pelvis) are transformed to a 2D spherical grid based on equation (6) and (7). For every triangle in SL table, every vertex is matching to a unique vertex in 2D spherical grid. Each combination of the two angle parameters $\theta$ and $\varnothing$, in the spherical coordinate system (Figure 3.12) represents a unique ray in 3D Cartesian coordinate system. With one more parameter; distance $d$, they produce a one-to-one correspondence to a vertex $(\mathrm{x}, \mathrm{y}, \mathrm{z})$ in the Cartesian coordinates system. Therefore, each vertex $(\mathrm{x}, \mathrm{y}, \mathrm{z})$ in Cartesian coordinates corresponds to a unique vertex $(\Theta, \varnothing, d)$ in spherical coordinates.

$$
x=d \sin \phi \sin \theta ; y=d \cos \phi ; z=d \sin \phi \cos \theta
$$

Where $d \in[0, \infty), \theta \in[0,360)$ and $\phi \in[0,180]$. 


$$
d=\sqrt{x^{2}+y^{2}+z^{2}} ; \theta=\tan ^{-1}\left(\frac{x}{z}\right) ; \phi=\cos ^{-1}\left(\frac{y}{d}\right)
$$

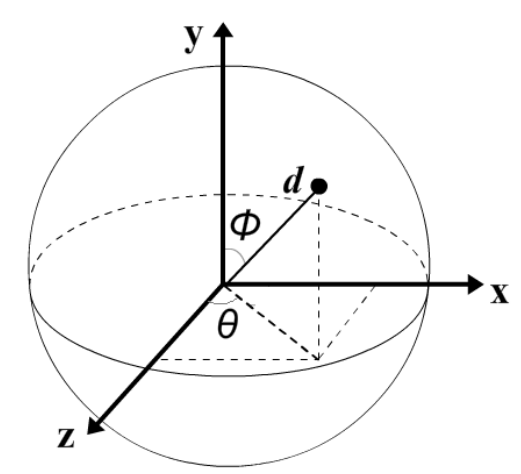

Figure 3.12: Spherical coordinate system.

Once the triangles in SL table are mapped to 2D grid, we rasterize all triangles to generate sample points. We can ensure the accuracy and efficiency of system with a rapid and wellorganized sampling method. With homogeneous and determinant sampling, we improve the computational time of collision detection as samples distance differences divulge the collision. Therefore, sampling procedure is vital for collision detection process.

Our sampling procedure is mainly based on rasterization and interpolation, but in order to achieve the homogenous and analogous sample points in two models we make some changes in rasterization step. The sampling method is modified as, we determine a tight bounding box for each mapped triangles in 2D spherical grid, as it contains all of the pixels in the triangle according to the predefined sampling precision $\left(0.05^{\circ}\right)$. This bounding box can simply be calculated based on the minimum and maximum of $\theta$ and $\varnothing$ of triangle vertices. Minimum and maximum of width and high of the bounding box are the nearest multiples of 0.05 to minimum and maximum of $\theta$ and $\varnothing$ of triangle vertices respectively (Figure 3.13). This ensures that the neighboring samples points of both models are placed in same orientations from HJC, with same 
distances between the points. Also, it guarantees that the sample points of both surface models are comparable.

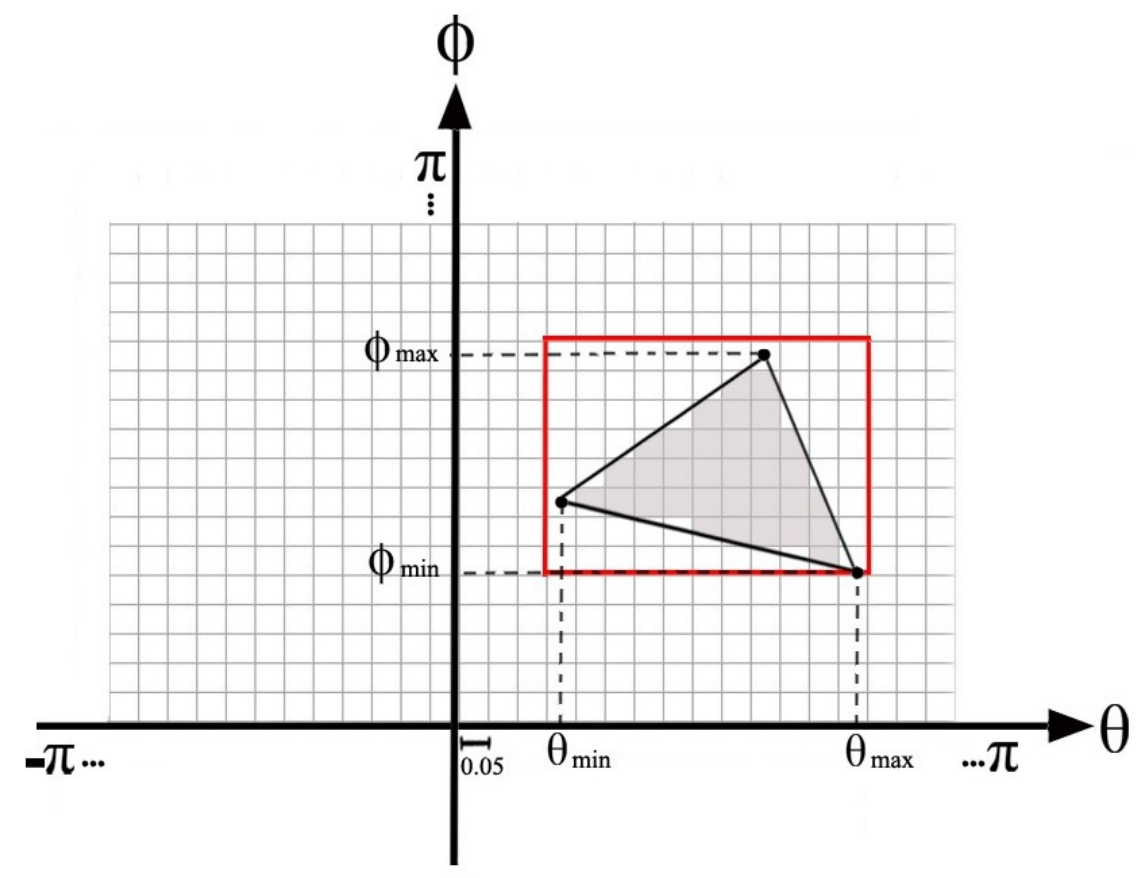

Figure 3.13: Triangle rasterization: a bounding box (red box) is fitted around each triangle in order to iteratively generate sample points by using rasterization and interpolation.

Once we identify the box, we loop over each pixel in the box, incrementing $\theta$ and $\varnothing$ by $0.05^{\circ}$ each time, and we compute the barycentric coordinates as the process of rasterization. New $\theta$ and $\varnothing$ values are the orientation parameters of generated sample points. Only those points are accepted that lie on the triangle primitives (face, edge, vertex). The barycentric coordinates also serve as interpolation coefficients. Distance $d$ of a pixel (representing the grey value) in a certain orientation $\left(\theta_{i}, \varnothing_{i}\right)$ is interpolated from the corresponding vertices of triangle from barycentric coordinates. 
A 2D LUT for each model is constructed which is indexed by spherical coordinate's $\theta, \varnothing$ (as ordinate and abscissa respectively) and filled by -1 value (denotes the cell is empty). Each time, the interpolated distance value $\left(d_{i}\right)$ of the sample point in the orientation $\theta_{i}, \varnothing_{i}$ is stored in the corresponding cell of LUT $\left(\operatorname{LUT}\left[\theta_{i}\right]\left[\varnothing_{i}\right]=d_{i}\right)$. On the other hand, there are some vertices and edges that are common between two or more triangles, so the algorithm discards those to prevent multiple calculations. The size of the LUT $[\theta][\varnothing]$ is considered as $25,927,200((360 / 0.05) *$ $(180 / 0.05+1))$, since the sampling precision is considered as $0.05^{\circ}$ degree. Once the sample points are generated and the LUT tables of both mesh model (proximal femur and pelvis) is filled with interpolated distance value; the distance difference between two surfaces as illustrated in Figure 3.11 are compared to ascertain the collisions and penetrations during movements.

If a model rotates, the new oriented sample points can be computed easily since the related positions between two models do not change and their geometric information are based on the HJC (which is also center of world).

Our experiment with two sampling methods shows that the spherical sampling of the surfaces based on rasterization is superior in term of speed and computational time. Also, it improves the efficiency and accuracy of collision detection as a result of uniformly sampling the surfaces and using a well-structured table like LUT to store the samples.

\subsubsection{Collision Detection Phase}

The sample points are generated for each surface mesh model and successively are stored in their LUTs. The next phase after sampling procedure is collision detection. Subsequently, the distance values of the samples points that approximate surfaces of both models are compared to ensure that there is a sufficient space between them as shown in Figure 3.14. So, the LUT of the 
acetabulum is compared to the LUT of the femoral head. Moreover, if a collision occurred then impingement zone is determined.
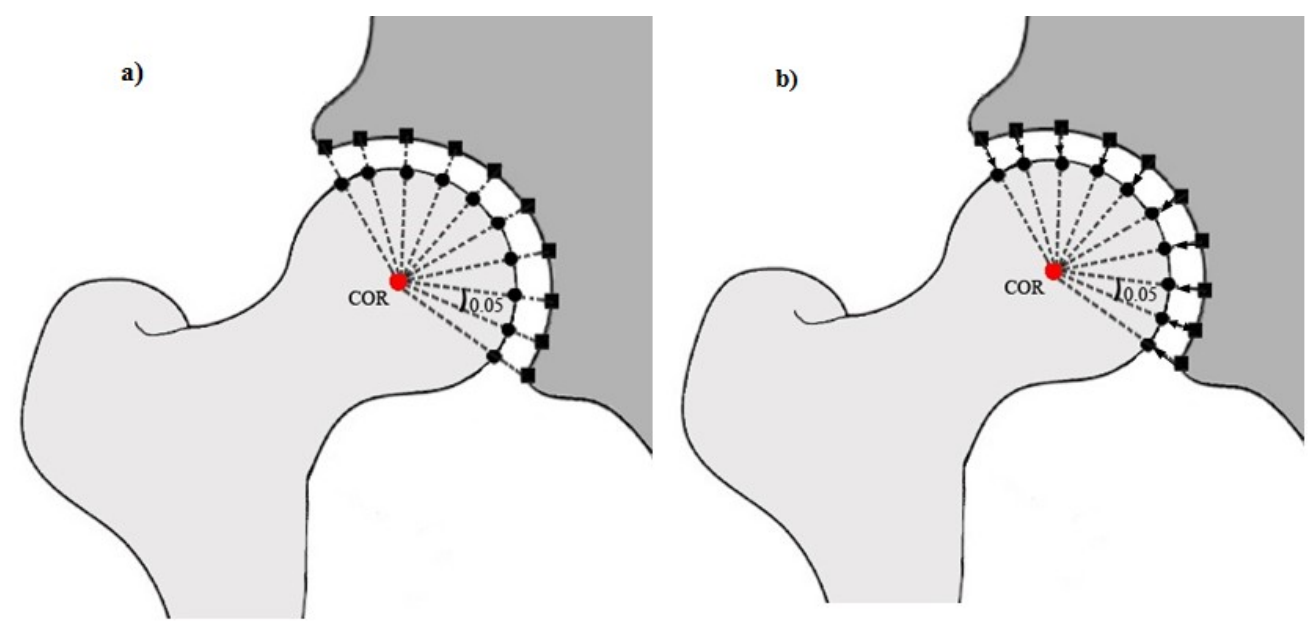

Figure 3.14: 2D conceptual representation of collision detection procedure: (a) samples point of each femoral head (black dots) and acetabulum (black square) surfaces are represented from HJC (red dot). (b) Distance difference between sample points of both models surfaces (black arrows) is computed.

Each cell of LUTs indicates a unique sample point in the surface which is distinguishable by its orientations $\theta, \varnothing$. We compute the distance diference between two sampled surfaces by comparing their LUTs cell in the corresponding indices. Since the LUTs of sample points are indexed same by $\theta, \varnothing$ and sample points for neighboring surfaces are congruent (same orientation); therefore comparing those LUTs provides us exact and correct detection of impingements. If a cell in an orientation is empty (-1), it means there is no collision in that direction. For those that are not empty, the distance differences of samples are compared. In general a constant distance between two mesh models is considered as the thickness of cartilage (2mm). If distance differences between two sampled surfaces become less than the cartilage 
thickness, it means that there is a collision and the models are penetrating. On the other hand, if the distances between two sampled surfaces are greater than the allowed distance, there is no collision between them. In overall, collision or impingement is recognized if one object is on or inside another object.

This collision detection algorithm, based on large sampling of surface mesh models and surface to surface distance measurement improves the accuracy and precision of the system. In addition, employment of a well-organized data structure like LUT optimizes the performance of collision detection procedure and simplifies its computation. On the other hand, it accelerates the detection of impingement in dynamic environment during hip motion as the sample points are rapidly generated and distance difference is computed in trivial amount of time. Generally, in a static position, it takes less than three seconds to sample the surfaces and detect the collision between models, regardless the number of sample points.

\subsection{Hip Motion Simulation Design}

In this section, we explain the design of our motion simulation system. A bony hip joint is simulated in 3D space in order to analyze and visualize hip maximal motions. The 3D bones of the joint are constructed from segmented MR images and their motions are simulated as that the femur rotates around the computed $\mathrm{HJC}$ which is center of the femoral head and about the arbitrary axes while the pelvis bone is fixed. In section 3.4.1, we simulate the motions of the hip joint at its extremity with the assumption of sharing a common center between the acetabulum and the HJC that is the center of rotation of the femoral head. Then, the ROM of the hip joint in each direction is calculated and visualized for further analysis. In this research, the ROMs of the hip joint represent the maximum possible rotational angles of the femur around the HJC. 
Generally, hip joint motions are restricted by bony morphology of the joint disregarding the effect of connective soft tissues factors since they are variable and flexible. Later on, we examine the possibility of $\mathrm{HJC}$ movement in direction of acetabulum center with the intention of maximizing the ROM in section 3.4.2.

\subsubsection{Simulation of Rotational Movements}

Hip joint motion is simulated as rotational movement in consequence of ball-and-socket joint and three DOF is considered due to articulated femoral head and acetabulum. As describe in section 2.1.2, normally, the hip joint undergoes six different rotational movements including flexion/extension in sagittal plane, abduction/adduction in frontal plane, and internal/external rotation in transverse plane. In our computer simulation system some combinational movement are designed rather than six main rotational movements.

After 3D construction of the proximal femur and the pelvis bone models from MR images,

they are registered in the system respectively as fixed and variable models. Then, the center of femoral head is calculated and considered as the HJC, as we explained in section 3.2. The coordinate systems of 3D proximal femur and pelvis bones are set according to the computed HJC as show in Figure 3.15. 


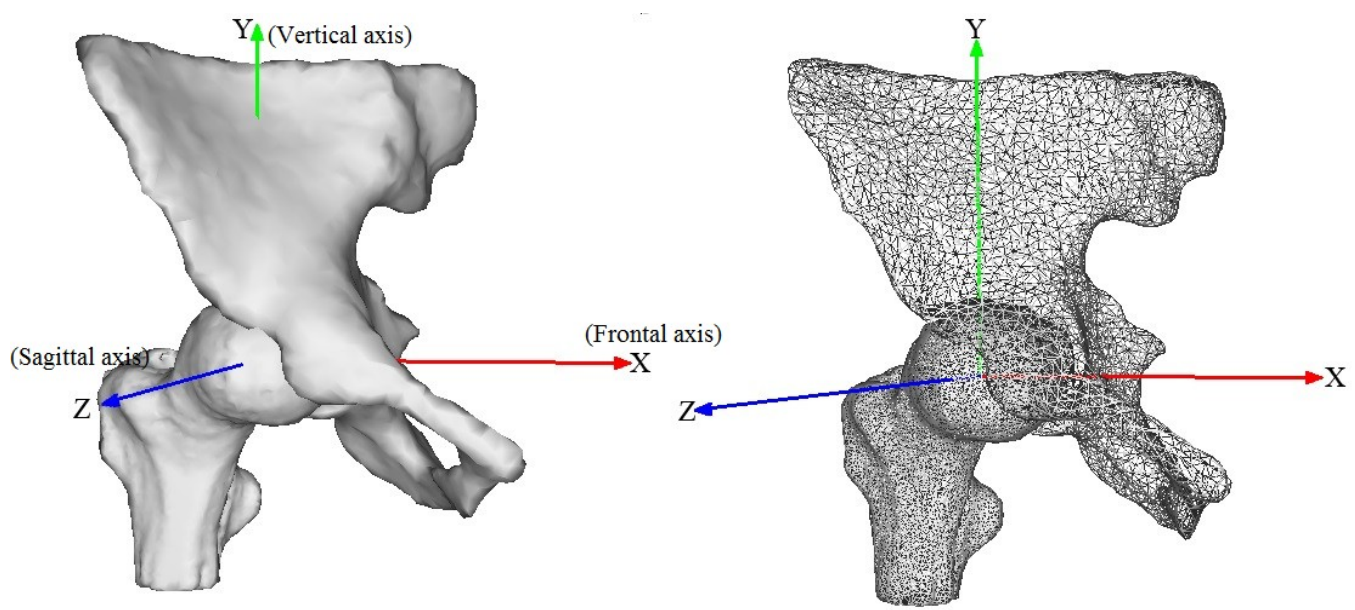

Figure 3.15: Illustration of coordinate system of $3 D$ proximal femur and pelvis bones based on HJC.

Consecutively, the proximal femur bone model is rotated around the calculated HJC within depression of the acetabulum around different axes while the pelvis bone model is fixed. The rotational movements of the hip joint, as illustrated in Figure 3.16, are conducted as rotation of the femur about the frontal (X-axis), sagittal (Z-axis) and vertical (Y-axis) axes in clockwise and counter clockwise (right-handed) to form the six main motions of the joint (Table 3.1).

Table 3.1: Anatomical reference axes and planes of hip joint rotational movements.

\begin{tabular}{|c|c|c|}
\hline Motion & Plane & Axis \\
\hline Flexion/Extension & Sagittal & Frontal \\
\hline Abduction/Adduction & Frontal & Sagittal \\
\hline Internal/External Rotation & Transverse & Longitudinal (Vertical) \\
\hline
\end{tabular}





Figure 3.16: Six main plane-movements of the hip joint: a)flexion b)extension, c)abduction, d) adduction, e) internal rotation, f)external rotation.

Each movement of the hip is restricted by the collision detection algorithm associated with bony morphological constraints. The digital proximal femur rotates around the HJC and stops whenever it detects collision. Collision detection, as described in section 3.3, is based on 2D spherical sampling of mesh models surfaces and surface to surface distance measurement. The sample points which are generated on the surface models in 2D spherical grid are stored in LUTs and then both LUTs are compared to ascertain the collision. During hip motion, the pelvis model is considered as fixed mesh model and it remains static. Therefore, we do not need to update the LUT of the acetabulum whenever a motion is applied. Moreover, the acetabulum position does not change related to the femoral head during rotational movement, since both surface models are centered on the HJC which is also center of world. 
Every time that proximal femur rotates, the system checks the distance between two bone models to examine if there is a collision between the bones or the distance between two models is less than the assigned uniform cartilage thickness $(2 \mathrm{~mm})$. The maximum possible rotation degrees of the proximal femur around the HJC is calculated for every direction about the rotational axes and considered as the ROM of the joint.
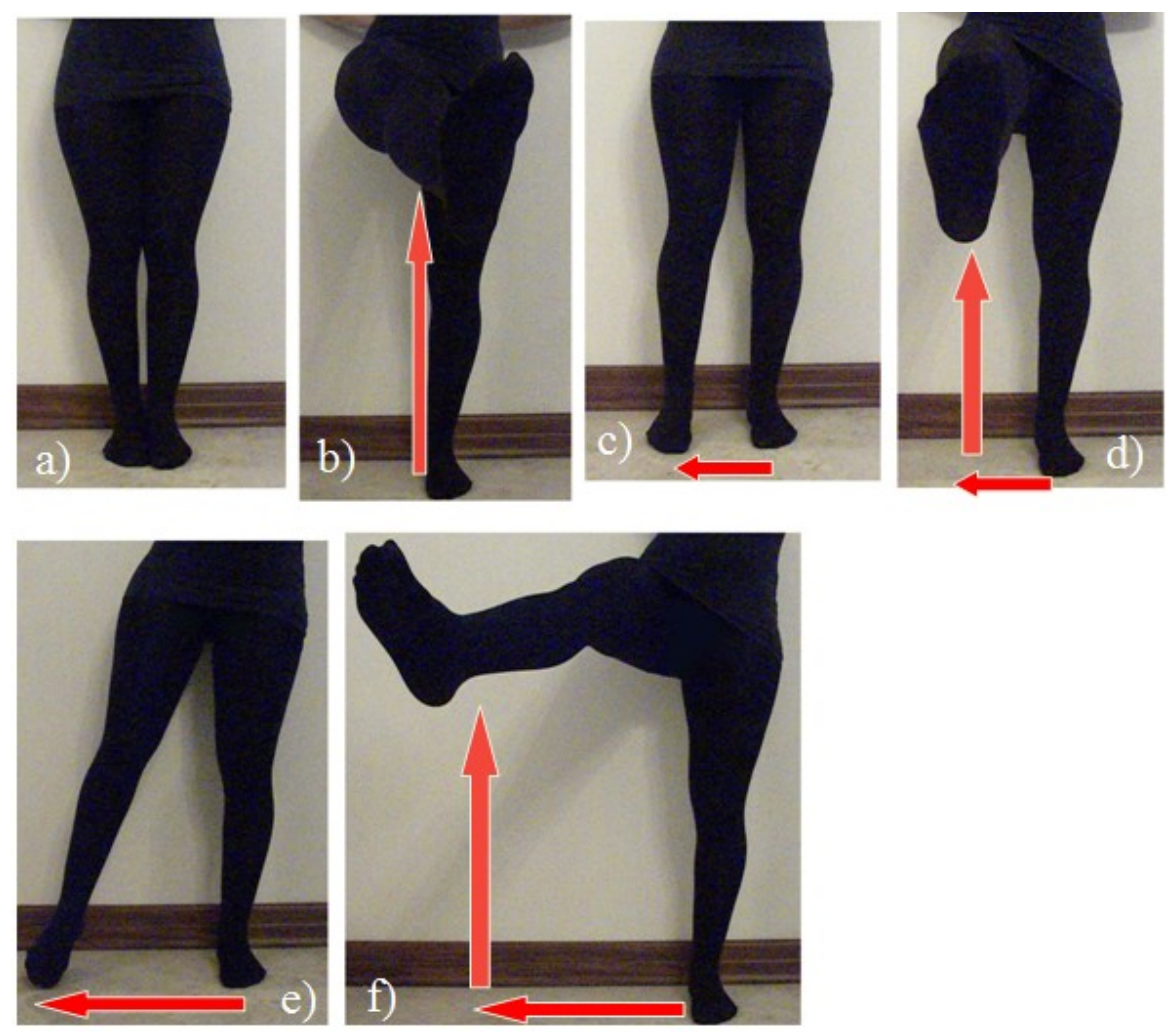

Figure 3.17 a-f) Illustration of successive movement; flexion movement per abduction movement.

a) Hip in neutral pose where the abduction degree is zero. $b, d$ and $f$ show the maximum flexion per abduction movement of a $\left(0^{\circ}\right), c\left(20^{\circ}\right), e\left(40^{\circ}\right)$, respectively.

In addition to six plane-movements of the hip, successive movements, as maximum rotational movement per degree of another rotational movement (e.g. maximum flexion movement per abduction movement) (Figure 3.17) and combinational movement of the hip joint are simulated. 
The successive movements of the joint are simulated to analyze flexibility and extendibility of the joint when it is abducted/adducted or internally/externally rotated. On the other hand, the combinational movement of the joint is simulated to analyze the maximum achievable rotational movement of the joint between every two main rotational movements like Figure 3.18.
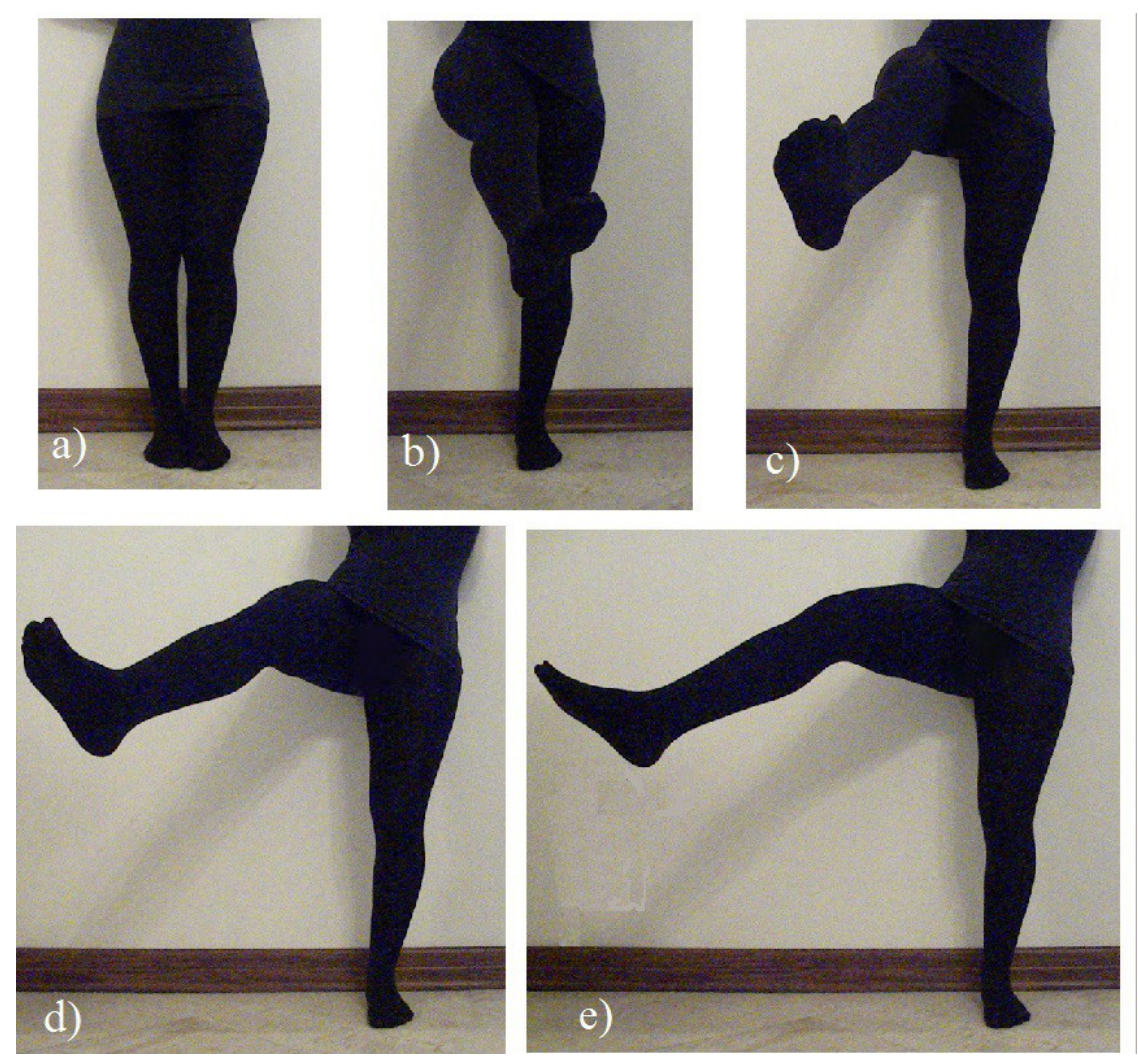

Figure 3.18. a-e) Illustration of combinational movement of the hip joint that shows the maximum rotational movement between flexion (b) and abduction (e) movement, around 12 to 3 clock hours hand ("a" shows the hip in neutral pose).

We simulate a combinational movement for the joint as rotation of the femur around the HJC about the rotational axes which are the clock hours hand as illustrated in Figure 3.19. A clock system is designed for the $3 \mathrm{D}$ femur bone in a way that, the face of the clock is projected on the transverse plane which is passed through the HJC as shown in Figure 3.19. The center of clock is 
placed at the HJC and divides the femoral head into 12 sectors. The 12 and 6 o'clock are placed on frontal axis and the 3 and 9 o'clock are placed on sagittal axes (Figure 3.19). After that, the maximum rotational movement of the joint is performed around the clock hours hand in clockwise, from 12-6-12 o'clock (right-handed). The maximum degrees that hip can rotate around each axis (clock hours hand); restricting by the collision detection algorithm, is considered as the maximum ROMs of that o'clock. The envelopes of these extreme movements are assessed and visualized in section 4.2. Hip ROM is analyzed during these movements to evaluate the bone morphology effects in the ROM degrees as preoperative surgical planning of hip replacement and also in assessment of hip extreme motion. The results of these simulations are presented in Chapter 4.


Figure 3.19: Illustration of a projected clock on the transverse plane (the black square) which is passing through HJC (noted that center of clock is placed at the HJC). The left snapshot is topview and the right is front-view. The axis $Z$ is the front of body and the axis $X$ is inward.

\subsubsection{Moving Hip Joint Center}

There is a long-standing question in hip joint study which is if the HJC is fixed or it is moving. Most of the studies in the area of motion simulation and collision detection treat the HJC as a 
fixed point. On the other hand, there are some researches which show the center of femoral head is not fixed and the rotation of the femur is associated with gliding movement as we mentioned in section 2.3.1.1. Therefore, there can be the possibility of improvement in hip mobility.

Through this research, the possibility of moving HJC is examined and the ROM variation is analyzed through our computer simulation system. In order to fulfill this goal, center of acetabulum is calculated for 3D pelvis bone model as shown in Figure 3.20. This calculation is based on best sphere fitting to four markers (control points) on its rim and depth as explained in section 3.3. Afterward, the HJC is moved inward and outward acetabulum in direction of acetabulum center and axes (Figure 3.21). Consequently, the local coordinate system of the femur is computed based on the new center. Successively, six main plane rotational movements of the hip is conducted around the new center and about the rotational axes of the acetabulum. During each rotational movement, the maximum degree of impingement-free motion is computed with the control of impingement detection system. Then, the maximum ROM degrees are computed for each movement from bony structure of the joint and compared with the ROM result of fixed center. 


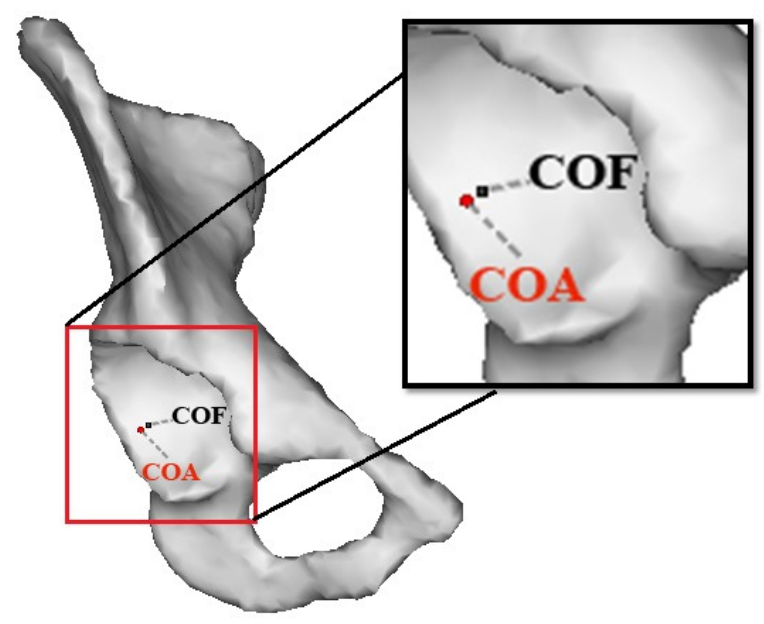

Figure 3.20: Illustration of center of the acetabulum (indicated as COA in red) compare with the HJC or center of the femoral head (indicated as COF in black).



Figure 3.21: Illustration of moving the HJC a) outward and b)inward the acetabulum. 


\section{Chapter 4. Result and Visualization of Range of Motion}

The system is developed with the aim of simulation and visualization of hip maximal motions from MRI. To achieve this objective, MRI data of the right hip of a 37 years old Caucasian male patient with the weight of $88 \mathrm{~kg}$ is acquired for our experiment. The resolution of MR image is 256 pixels by 256 pixels and the thickness of each slice is $0.81 \mathrm{~mm}$. While many works are developed in order to construct 3D models from medical data (CT or MRI), but our research focus is not on $3 \mathrm{D}$ construction. Hence, we do not further analysis $3 \mathrm{D}$ construction methods and we use ITK-SNAP 2.4.0 toolkit to segment our bone models. Therefore, One hundred and sixty MRI slices in format of DICOM are segmented by using ITK-SNAP toolkit. After that, our segmented images are verified with a medical doctor; a musculoskeletal radiologist, from Ottawa General Hospital. Successively, some preprocessing steps completed on the segmented MR images as discussed in section 3.4 and as the result, 3D mesh models of the proximal femur and the pelvis bones are constructed.

The complexity of obtained 3D triangulated meshes is reduced to 7943 and 37536 vertices for the femur and the pelvis, respectively. As the process of motion simulation, the HJC is estimated from the constructed bone models as section 3.2. Consecutively, different motions are conducted which are restricted by a collision detection algorithm according to morphology of the bones (section 3.3). ROM for each movement is computed and the envelope of the motion is visualized. 


\subsection{Range of Motion}

\subsubsection{Six Main Plane-Movements}

Through our computer-based motion simulation system, the upper limits of rotational angles for pair of plane-movements (flexion/extension, internal/external rotation, and abduction/adduction) about different axes are evaluated as shown in Table 4.1, for 3D constructed bones of the adult male after segmentation of MRI. The ROM values represent the maximal rotational degrees of the hip movements that are constrained by the joint morphology. These degrees are the permitted relative motion of the hip joint. Moreover, the impingement zones during flexion and abduction are illustrated in Figure 4.1 and Figure 4.2 , respectively.

Table 4.1: Our result of rotational degrees for six extreme plane-movements of right hip around a fixed HJC.

\begin{tabular}{|c|c|}
\hline Motion & Ranges ( ${ }^{~}$ ) \\
\hline Flexion & 119.8 \\
\hline Extension & 59.4 \\
\hline Abduction & 38.96 \\
\hline Adduction & 36.03 \\
\hline Intemal Rotation & 35.06 \\
\hline External Rotation & 30.2 \\
\hline
\end{tabular}




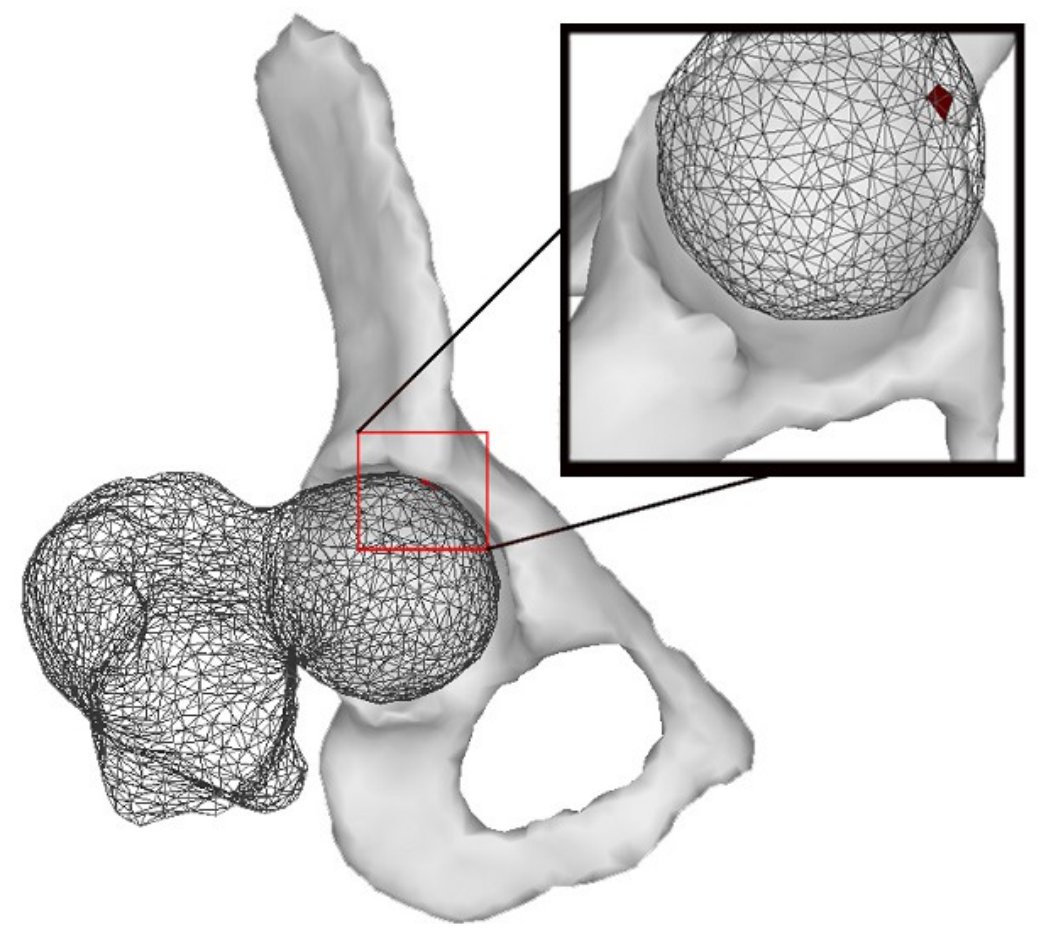

Figure 4.1: Illustration of impingement zone (red color) during flexion movement.

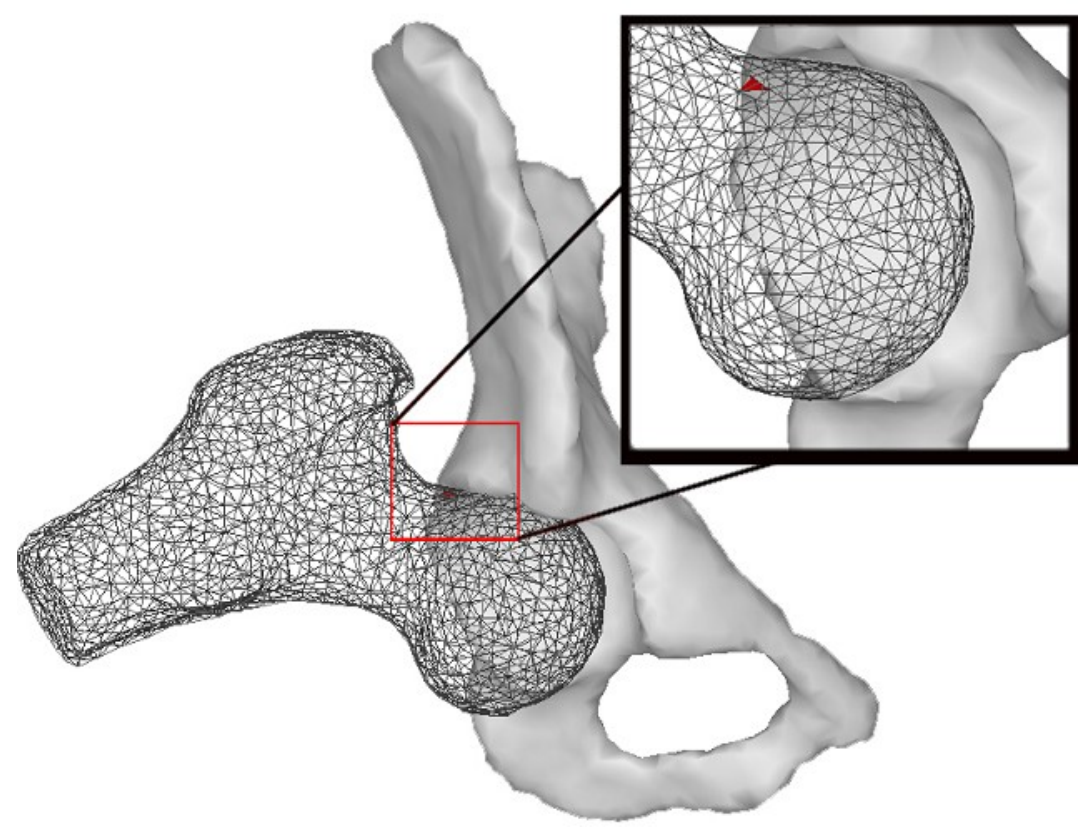

Figure 4.2: Illustration of impingement zone (red color) during abduction movement. 


\subsubsection{Successive Movements}

Four pairs of successive motions are carried out, adduction with flexion and extension and also abduction with flexion and extension (Figure 4.3, Figure 4.4). These graphs demonstrate that how ROMs of flexion and extension movements are changed in related to abducted and adducted hip joint. It can clearly be seen that both graphs show descending trends. The abducted and adducted hip show less flexibility and extendibility compared to neutral pose. Figure 4.3 depicts that flexion rotational angles are declined moderately, down to $54.55^{\circ}$ with increasing abduction to $35.07^{\circ}$ degree and then are slowly increased to $61.36^{\circ}$ degree at the maximum abduction rotational angle. While, the extension rotational degrees are remained steady with abduction up to $25.32^{\circ}$ and then is decreased roughly to about $1^{\circ}$ degree with a fluctuating trend.



Figure 4.3: Maximum rotational angles $\left({ }^{\circ}\right)$ of flexion and extension in diverse abduction degrees. 
On the other hand, with increased adduction angles flexion degrees are slightly decreased to approximately $105^{\circ}$ and extension degrees are steeply dropped to about $1^{\circ}$ degree. In extension movement, this decrease is considerably but in flexion, firstly range is approximately peaked to $125^{\circ}$ in $5^{\circ}$ degree of adduction and then declined slowly (Figure 4.4).

By and large, the flexibility and extendibility of the joint is diminished by continuously abducting or adducting the hip and this decrease is noticeably for extension movement.

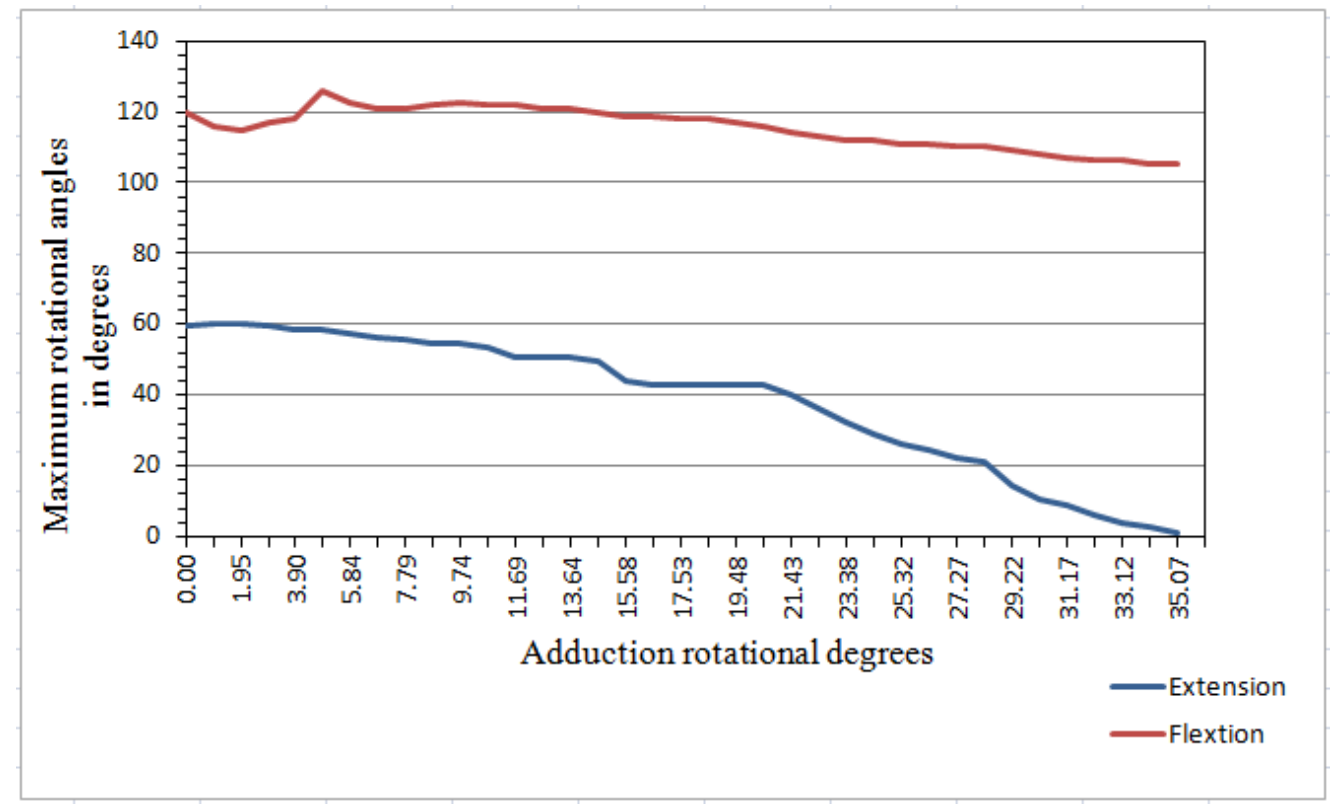

Figure 4.4: Maximum rotational angles $\left({ }^{\circ}\right)$ of flexion and extension in diverse adduction degrees.

In addition, two successive motions; external and internal rotation with flexion, are performed to evaluate the flexibility of internally and externally rotated hip. Figure 4.5 shows that with increasing internal and external rotational angles, the flexibility of the hip is reduced with almost similar trends. When the external rotational angle amplified, flexion rotational angles firstly fluctuate approximately between $105^{\circ}$ to $120^{\circ}$ in seventeen degrees of external rotation and then from $17.5^{\circ}$ to $18.5^{\circ}$ degrees of external rotation, it is decreased sharply to $25^{\circ}$ and is moderately 
sank to about $2^{\circ}$ in maximum external rotational angle, with a plateau of flexion between $19.48^{\circ}$ and $23.4^{\circ}$ degrees of external rotation. In contrast, with increase in degree of internal rotation to $4^{\circ}$ degree, the maximum flexion angle is remained stable and then it is decreased a bit and is remained unchanged again. From $10.7^{\circ}$ to $25.3^{\circ}$ degree of internal rotation, the flexion degree goes up slowly to $125^{\circ}$. In $26^{\circ}$ degree of internal rotation, flexion degree is plunged to $18.5^{\circ}$ and decreased gently all over to $5.8^{\circ}$ in the maximum degree of internal rotation (Figure 4.5).

Overall, Figure 4.5 shows that flexibility of hip joint is diminished by rotating the joint internally or externally.

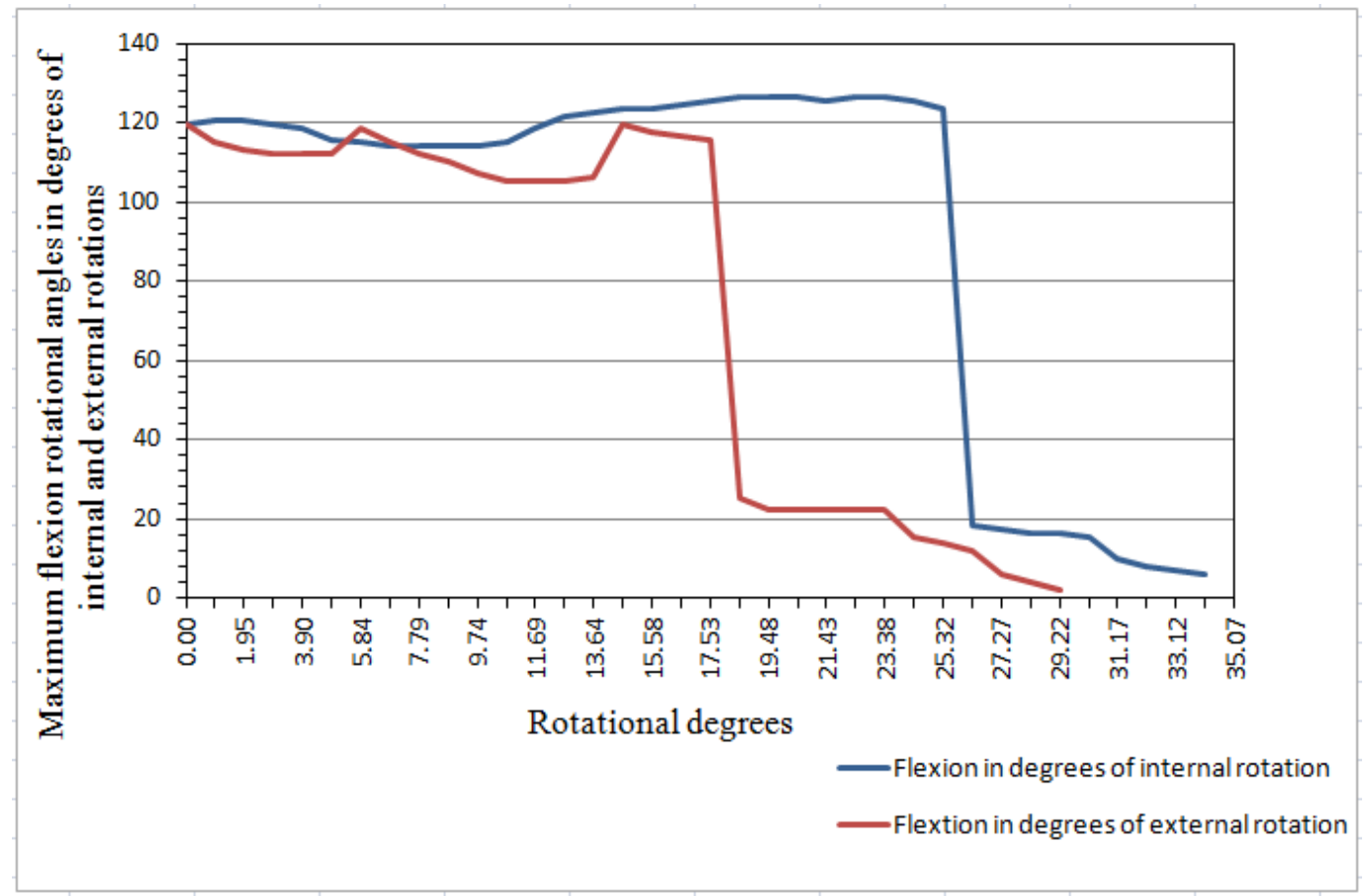

Figure 4.5: Maximum flexion rotational angles (degrees) of hip joint in degrees of external and internal rotations. 


\subsubsection{Combinational Movement}

Furthermore, a combinational movement is performed so that a clock is projected, as shown in Figure 3.19, on the transverse plane passing through the center of the femoral head (HJC) in the way that it divides the femoral head into 12 sectors corresponding to the clock face and the center of clock is at the HJC. The 12 and 6 o'clock are placed on frontal axis and the 3 and 9 o'clock are placed on sagittal axes. The maximum rotational movement of the joint is performed around the clock hours hand in clockwise (right-handed). As shown in Figure 4.6, the degree of rotational angles, from 12 to 12.8 o'clock is decreased to $96.4^{\circ}$ and then it is climbed to a peak of $131.5^{\circ}$ degree at 1.4 o'clock. From 1.4 o'clock to 3.8 o'clock, the maximum rotational degree is dropped quickly. At 3.8 o'clock, the trend is reached the lowest degree which is $31^{\circ}$. From 4 o'clock, the maximum rotational degree begins to climb again until 5 o'clock and then the degree is dropped gradually until 9'oclock and after that it is enlarged again to $128.5^{\circ}$ at 11.8 o'clock with a slow change at 10.4 o'clock and 11.8 o'clock. Hip joint performs flexion, abduction, extension and adduction movements around 12,3,6 and 9 o'clock, respectively. 


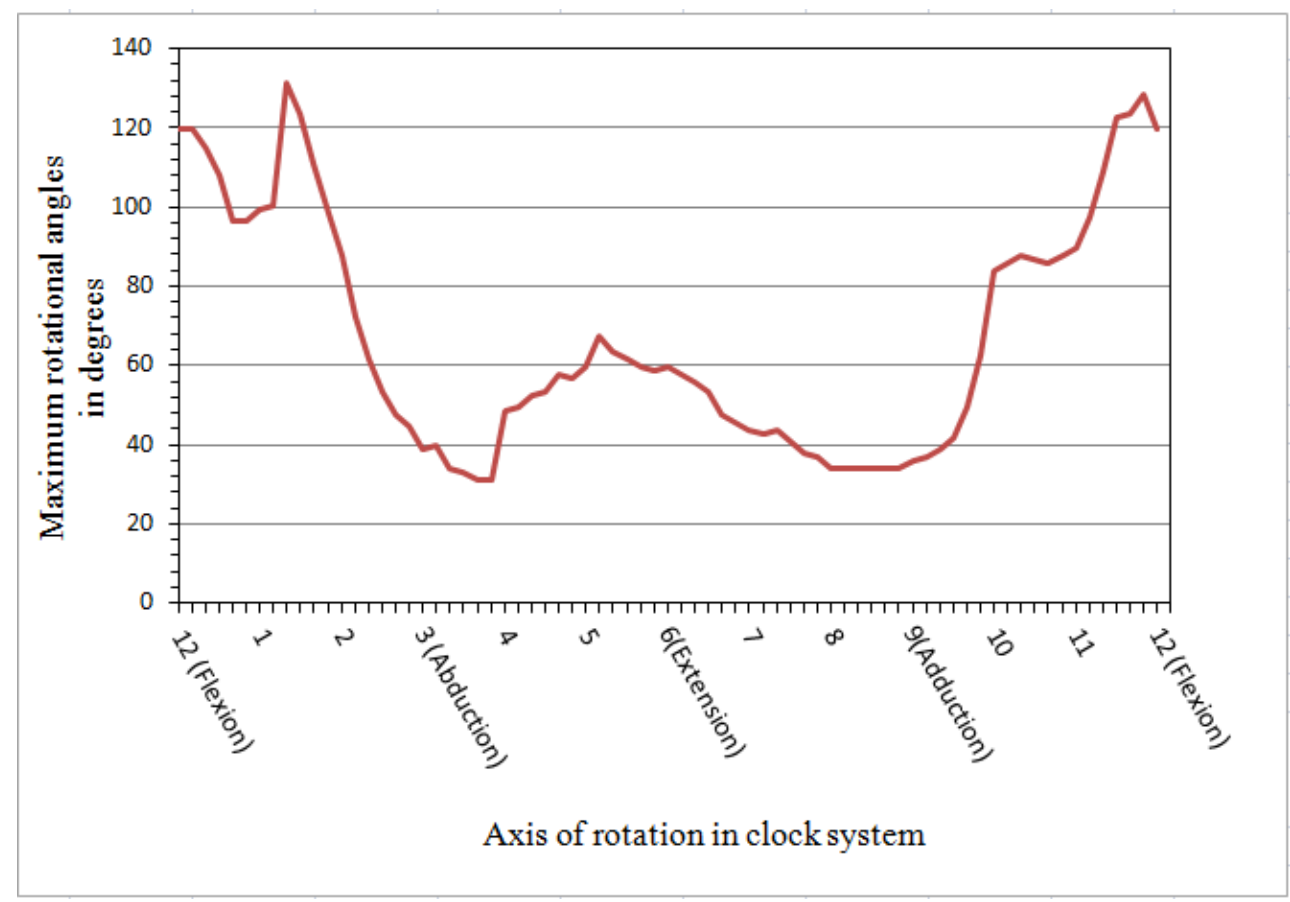

Figure 4.6: Maximum rotational angles $\left({ }^{\circ}\right)$ of hip joint around clock hours hand when the clock face is projected on the transverse plane that is passing through the HJC (center of clock is placed at the HJC).

\subsection{D Visualization of Range of Motion}

After simulation of hip joint motion, the envelopes of motions are visualized in 3D space. The femur in the neutral pose is assumed as the position of a line passing from the HJC to a point with coordinate data $1.0 \mathrm{~cm}, 2.0 \mathrm{~cm}$ and $0.0 \mathrm{~cm}$, respectively as $\mathrm{x}, \mathrm{y}$ and $\mathrm{z}$ values. The maximum ROM degrees are applied to this line to illustrate the envelope of the maximal movements of the hip joint which is computed from bony morphology of the joint. The envelope of aforementioned combined motions is visualized in (Figure 4.7). The computed ROMs from Figure 4.6 are applied to this line to illustrate the envelope of this combined motion. 


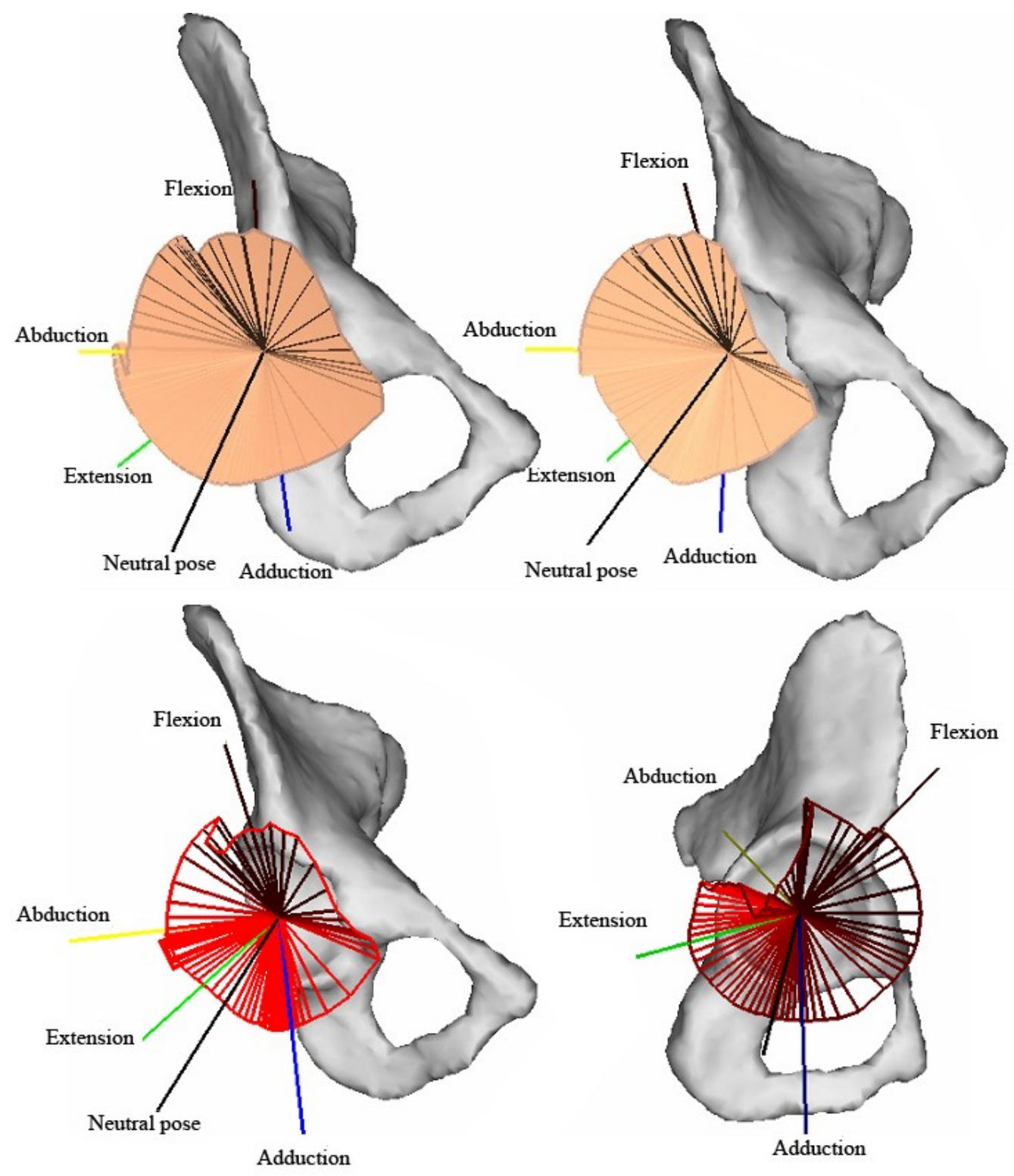

Figure 4.7: Envelope visualization of Figure 4.6 which represents hip combinational movements. Top row is colored representation of this envelope and bottom row is wired representation of the envelope, from different views.

The envelope of a successive movement is illustrated in Figure 4.8 which represents the flexibility and extendibility of the joint per abduction movement as demonstrated in Figure 4.3. 
The computed ROMs of Figure 4.3 are applied to the line presenting the femur in its neutral pose and the following envelope is obtained.


Figure 4.8: Envelope visualization of Figure 4.3 representing hip successive movements;

abduction with flexion and extension. Blue envelope represents the extendibility of the hip joint per abduction movement while the red envelope represents the flexibility of abducted joint (yellow line shows when the hip is abducted only).

These envelopes would be influenced by FAI in a way that it will be shrunken with the presence of FAI compared with a healthy patient's motion envelope. Motion envelope of a patient diagnosed with FAI can be compared for before and after the corrective hip surgery to qualify the FAI treatment. In addition, these envelopes gives us a better understanding of how far the hip can move inside the acetabulum and also it provides us a framework to measure and analyze the rotational angles of different hip bone shapes. For instance, these envelopes would be enlarged for a hip with more rounded femoral head. 


\subsection{Moving Center Experiments}

In this research, we examine the effect of moving HJC on the maximum ROMs for the main six plane-movements of the hip joint. To achieve this goal, the center of 3D bone model of the acetabulum is calculated based on the best sphere fitted to the articulated portion of acetabulum as described in section 3.3. According to our achieved result, there is a gap between the centers of the femoral head (HJC) and the acetabulum. The coordinates of acetabulum center are calculated with respect to the $\mathrm{HJC}$ as $-1 \mathrm{~mm},-0.7 \mathrm{~mm}$ and $0.4 \mathrm{~mm}$ (where radius of sphere fitted to acetabulum is $15.12 \mathrm{~mm}$ ) in $\mathrm{x}, \mathrm{y}$ and $\mathrm{z}$ dimensions, respectively. Therefore, we examine the possibility of moving the HJC in direction of the acetabulum center outward (Table 4.2) and inward (Table 4.3) the acetabulum depression. To evaluate the effect of moving center inward the acetabulum on the maximum ROMs, the $\mathrm{HJC}$ is moved in direction of acetabulum center axes to $0.25 \mathrm{~mm}, 0.175 \mathrm{~mm}$ and $0.1 \mathrm{~mm}$ in $\mathrm{x}, \mathrm{y}$ and $\mathrm{z}$ dimensions.

Table 4.2: Maximum ROMs of six plane-movements of hip joint when the HJC is moved to the acetabulum center, outward the acetabulum depression compared with fixed HJC (center of femoral head).

\begin{tabular}{|c|c|c|}
\hline \multirow{2}{*}{ Motion } & \multicolumn{2}{|c|}{ Maximum ROM( ${ }^{\circ}$ ) } \\
\hline Flexion & Fixed HJC & Moved HJC Outward Acetabulum \\
\hline Extension & 119.8 & 142.2 \\
\hline Abduction & 59.4 & 86.69 \\
\hline Adduction & 38.96 & 48.70 \\
\hline Internal Rotation & 36.03 & 40.9 \\
\hline External Rotation & 35.06 & 59.4 \\
\hline
\end{tabular}


Table 4.3: Maximum ROMs of six plane-movements of hip joint when the HJC is moved to the acetabulum center, inward the acetabulum depression compared with fixed HJC.

\begin{tabular}{|c|c|c|}
\hline \multirow{2}{*}{ Motion } & \multicolumn{2}{|c|}{ Maximum ROM( ${ }^{\circ}$ ) } \\
\hline Flexion & Fixed HJC & Moved HJC Inward Acetabulum \\
\hline Extension & 119.8 & 67.21 \\
\hline Abduction & 59.4 & 23.38 \\
\hline Adduction & 38.96 & 31.17 \\
\hline Internal Rotation & 36.03 & 20.45 \\
\hline External Rotation & 35.06 & 13.64 \\
\hline
\end{tabular}

According to our observation, the ROM degrees are decreased with moving the center inside the acetabulum and there are significant rises when the HJC moves outward the acetabulum (Figure 4.9).



Figure 4.9: Comparison between the effects of moving center on the maximum ROMs. 


\subsection{Discussion}

Reflecting upon the results are obtained in this study, further important discussion can be drawn.

\subsubsection{ROM Analysis}

Different plane-movement, successive and combined hip joint motions are simulated and visualized and the ROMs are analyzed. Moreover, the envelopes of one combined movement and one successive movement are visualized and further analyzed. In this research, all the conducted motions are the maximum achievable motion of the joint, based on the bony morphology of the femur and the acetabulum.

\subsubsection{Successive Movements}

Relying on our observation on conducted successive motions, maximum flexion and extension rotational angles are considerably reduced per abduction and adduction motions. However, this reduction is more significant for extension movement rather than flexion. The extendibility of abducted and adducted hip joint is declined to almost zero degree at the maximum degrees of abduction and adduction. But the result shows that there is still possibility of flexion when the joint is abducted and adducted. On the other hand, per rotation of the hip internally or externally, the flexion remained steady at first and then in two different points, the flexion degree is dropped. In overall, it is observed that the flexibility of hip joint is diminished by rotating the joint internally or externally. 


\subsubsection{Combinational Movement}

The combinational movement of the hip is designed as rotation of the femur around the HJC about the rotational axes which are the clock hours hand of the projected clock on the transverse plane. Flexion, abduction, extension and adduction are occurred at rotation around 12, 3, 6 and 9 o'clock, respectively. As we expected, the maximum rotational degree is increased between flexion and abduction and the peak of maximum rotation is happened between 12 o'clock to 3 o'clock and then it is declined when the abduction is occurred. Around 3.8 o'clock, the trend is reached the lowest degree of rotation. Subsequently, the degree is increased slowly between 3.8 o'clock and 6 o'clock when extension is occurred and between 6 o'clock and 9 o'clock again is declined until the adduction is occurred. Towards the end, from 9 o'clock to 12 o'clock (adduction to flexion), the rotational degree is increased. Although these ROMs may vary for different bone shapes, we expect to see similar trend for all type of the normal bone shapes.

\subsubsection{Difference between ROM of Healthy Hip and FAI Patients Hip}

A hip joint with diagnosing to FAI may show different trends due to osseous abnormality of the joint. Therefore, the joint with FAI can be distinguished from healthy hip. The visualization of ROMs envelopes can presents a better perspective of the effect of FAI variety on ROMs extents, as well as, the effect of bone shapes of the joint on the ROMs. However, at this thesis, due to lack of data, we don't evaluate the ROMs of a patient with FAI. As our future work, more data would be collected and envelopes of these motions for healthy hips and patients diagnosed with FAI would be compared and analyzed. 


\subsubsection{Moving Center}

In this research, the effect of moving center on the ROMs is evaluated. Based on our experiment, the hip center may move outward the acetabulum to maximize the ROM but when the HJC is moved inward the acetabulum, the ROMs degrees are decreased. Therefore, we can conclude that considering the hip joint as ball-and-socket joint will give more flexibility to the joint than a conchoid joint. The research which is done by Yazdifar et al. [36] confirms our result about the effect of moving center on the kinematic of the hip joint and the ROMs. They examined their research for the CT scan of a patient with Cam FAI. They monitored the effect of center of rotation displacement on the kinematics of the joint with FAI, based on the captured motion data. On the contrary, in our motion simulation and visualization system, we examine the effect of moving HJC on the maximum achievable motions of the hip according to the shape of the bones (which are constructed from MRI) through a computer simulation rather than a motion capture system. Therefore, we minimize the errors that may happen during motion capture procedure which we discussed in section 2.3.2 and also the risk of CT scans due to their high exposure of radiations. Thereby, our versatile markerless digital simulation of hip joint motions allows the process to be easy and improves accuracy and efficiency of the system. In addition, our system simulates the motions that are restricted by an impingement detection algorithm and all types of impingements can be taken into consideration including intra-articular, posterior and superior articular impingements.

\subsubsection{Collision Detection System}

Our impingement detection algorithm examines the distance between the proximal femur and the acetabulum to prevent the impingement (collision) between two bone models during motion. 
compare to Cai et al. [33] approach which impingement detection processed twice for the static models, the femoral head's vertices tested against the acetabulum LUT as the first scan and then the acetabulum vertices tested against the femoral head's LUT as the second scan, we performed the impingement detection test once by comparing the LUTs together since they are indexed same (sampling precision of $0.05^{\circ}$ ). Furthermore, compared to the sampling approach proposed by Sik [73], which is based on randomly generation of the sample points, our sampling approach would speed up the collision detection process and beside requires a smaller amount of memory by use of LUT. Moreover, our sampling method is independent of complexity of mesh models, since a constant number of sample points is generating for each surface model and even for the less complex mesh models, we can still get the result with high precision and accuracy. The immense advantage of our method is its extendibility to entail the cartilage thickness information as the thickness of cartilage is varying and irregular. Additionally, since the impingement detection is based on distance difference, we can compute how close the surfaces are with providing the proximity information of the surfaces (femur and acetabulum).

Furthermore, our emphasis is placed on the investigation of osseous impingement, for example, the acetabular labrum is not considered during detection of impingement. However, the system has flexibility to add such information to improve its functionality and reliability.

\subsubsection{Sphere or Conchoid}

In light of these findings, some limitations of the present study have to be discussed. First, the HJC is calculated based on the center of sphere fitted to the femoral head. However, some studies are done about the HJC that shows the femoral head represents better a conchoid shape rather than a sphere and the HJC is not fixed as it is mentioned in section 2.3.1.1. But we have 
not calculated the rotation center of conchoid as its motion is non-determined. Therefore, at the current stage of the research, we calculate the HJC as center of femoral head as a common solution and we evaluate the possibility of moving the center.

\subsection{Validation}

All the experiments and tests of our hip joint motion simulation and visualization system are performed on the same and modern desktop computer (Intel i7 processor with 4 cores running 2.67 GHz, 6GB of DRR3 RAM running at Windows 7 64bit Operating System). The system is developed by using Microsoft Visual $\mathrm{C}++$ 2010. The simulation and visualization are implemented with OpenGL® (Open Graphics Library) and OpenCV® (Open Source Computer Vision Library).

Our system simulates and visualizes the maximum motions of 3D hip joint that are constructed from MRI after segmentation. The segmented bones are verified by medical doctors of Ottawa General Hospital. As the process of motion simulation, the HJC is estimated from the constructed femur bone model through our sphere fitting algorithm using Hough Transform method as explained in 3.2. In Figure 4.10, a color scale is used to visualize the difference from the calculated sphere to the surface of $3 \mathrm{D}$ femoral head. 

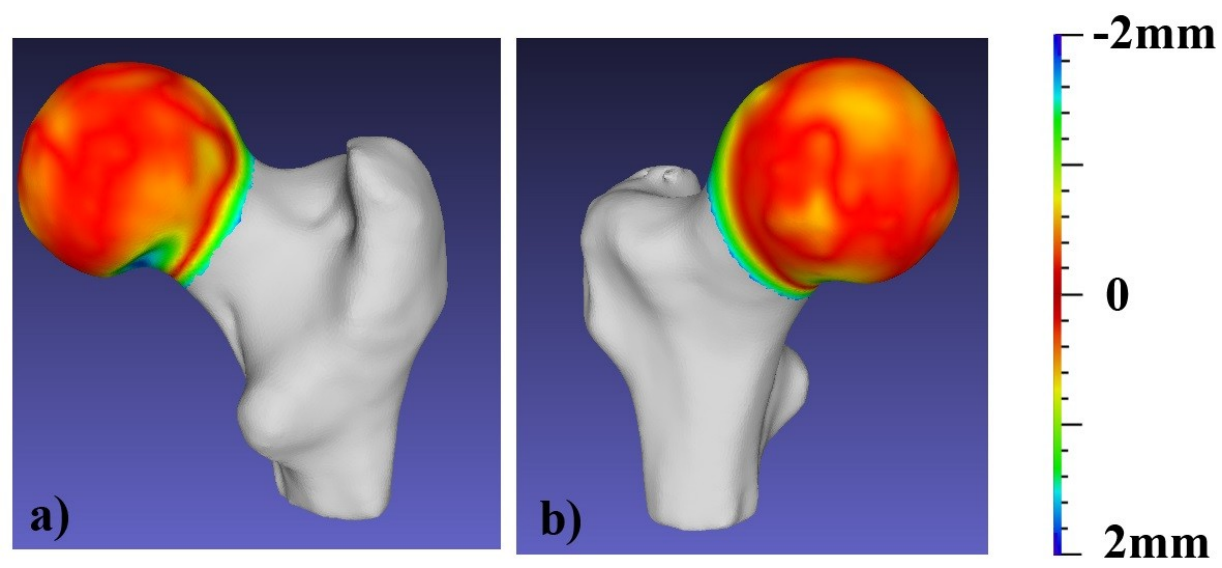

Figure 4.10: color scale of the difference from femoral head surface and fitted sphere.

Figure 4.10 shows that the error of fitting a sphere to the femoral head surface is minor and also Table 4.4 shows very small quantitative error. Therefore, such information demonstrates that the sphere is being well fitted to the $3 \mathrm{D}$ bone of the femoral head and proves robustness of the system in calculation of HJC.

Table 4.4: Quantitative errors obtained from difference between femoral head surface and fitted sphere.

\begin{tabular}{|c|c|}
\hline Distance & From Sphere to the Femur (mm) \\
\hline Min & 0.000000 \\
\hline Max & 0.49953 \\
\hline Mean & 0.06316 \\
\hline
\end{tabular}

After calculation of $\mathrm{HJC}$, the maximum motion of the joint is simulated with association of a robust and fast collision detection algorithm which is based on two main phases; sampling of surfaces and collision detection (section 3.3). Table 4.5 shows the computational time of our collision detection algorithm. 
Table 4.5: Performance analysis of our collision detection algorithm.

\begin{tabular}{|c|c|}
\hline Process & Computational Time in seconds \\
\hline Sampling phase & $1.3 \mathrm{~s}$ \\
\hline Collision Detection phase & $2.5 \mathrm{~s}$ \\
\hline
\end{tabular}

The exact quantitative validation of our motion simulation and visualization system is not possible, since the maximum ROMs are only calculated based on the bony structure of the joint and we do not consider the effect of other connective soft tissues like fats and muscles, since our objective is to evaluate the maximum achievable movement of the joint. Therefore, our calculated ROMs are different from those that can be determined during physical examinations as active and passive range of movements. Furthermore, based on the fact that ROM depends on the age, gender, ethnicity and geographic location (Table 2.2), it is difficult to ascertain an allencompassing set of hip ROM values. However, Table 4.6 shows our ranges are logical and accurate Comparing with normal ranges and the mean active ROMs of white men with ages 2539 years. In other words, we found the global behavior of bone ROM matches motion captured ROM with our experiment on a real person's data.

Moreover, ROM values are verified with the medical doctors from Ottawa General Hospital; musculoskeletal radiologist and orthopedic surgeon. 
Table 4.6:Evaluation of our computed ROMs with the normal ranges [14] and mean active range of motion for white men with ages 25-39 years [7].

\begin{tabular}{|c|c|c|c|}
\hline \multirow{2}{*}{ Motion } & \multicolumn{3}{|c|}{ ROM (') } \\
\cline { 2 - 4 } & $\begin{array}{c}\text { Normal } \\
\text { ranges[14] }\end{array}$ & $\begin{array}{c}\text { Mean ranges for } \\
\text { white men } \\
\text { (25-39 yrs) in } \\
\text { real-life[7] }\end{array}$ & $\begin{array}{c}\text { Our result } \\
\text { of bone } \\
\text { simulation }\end{array}$ \\
\hline Flexion & $0-125$ & 123 & 119.8 \\
\hline Extension & $115-0$ & 22 & 59.4 \\
\hline Abduction & $0-45$ & 46 & 38.96 \\
\hline Adduction & $45-0$ & - & 36.03 \\
\hline Internal Rotation & $0-45$ & 34 & 35.06 \\
\hline External Rotation & $0-45$ & 33 & 30.2 \\
\hline
\end{tabular}




\section{Chapter 5. Conclusion}

In this chapter, we summarize and conclude the works are developed and some gained insights within this dissertation. We also outline our key contributions and point out the potential and future work direction.

\subsection{Summary}

In this research, we present a computer-assisted simulation system to simulate and visualize hip joint motion from MRI of the patients. Through the system, 3D surface of the proximal femur and the pelvis bones are constructed from MR Images that are manually segmented with verification of medical doctors of Ottawa General Hospital. Then, the hip joint is considered as a ball-and-socket joint and its rotational movement is performed around a fixed HJC, which is considered as center of the femoral head and it is computed from best sphere fitting to the sphere portion of the femoral head. Hip joint motion is designed as the maximum potential movement of joint restricted by its bony structure without considering the connective soft tissues effects such as muscle and fat, since they are variable factors and they might show bigger ROM after taking exercise or physical training. In other words, our simulated motions exhibit extreme motions that are assessed depending on bone morphology of the hip joint.

The system simulates diversity of single, successive and combined hip joint motions that are controlled by a fast collision detection algorithm. During motion simulation the proximal femur rotates around the $\mathrm{HJC}$ about an arbitrary axis and stops whenever it detects collision while an equidistant distance is considered between models as cartilage thickness. The maximum ROM 
degree of each conducted motion is computed through our effortless and painless motion simulation system with less error in comparison with motion capture systems in real-life. Therefore, our motion simulation system can eventually be used before and after surgery to evaluate the effect of the applied osseous repairs on the maximal ROM degrees based on the MRI of the patient which is diagnosed with FAI. Furthermore, since the computed ROM degrees are extracted from bone shape of the joint, these degrees represent that if the patient (dancer or athlete) undertake the exercises that are involving the motions greater than these ranges, then there would be the possibility of FAI for the patient. Even for non-athlete patients, by analyzing the ROMs from bony morphological shape of the joint, the system determines whether there is a possibility of ROM improvement by undergoing physical training or not.

In addition, the 3D envelopes of the maximum ROMs are visualized. Our 3D visualization system of the hip joint motion helps to improve communication of patient and doctor by providing more comprehensible representation of hip mobility through 3D representation of motion envelope. Our proposed motion simulation and visualization system was welcomed by the medical doctors, particularly Orthopedics surgeons, from Ottawa General Hospital.

Furthermore, in our research the possibility of moving HJC is examined. It is observed that moving the center of femur in direction of acetabulum center, outward the acetabulum will improve the ROM of the joint but inward the acetabulum will result to ROM reduction. 


\subsection{Contribution}

Our contributions can be itemized as follows:

- We developed a computer-assisted simulation system to compute the maximum range of impingement-free motions from MRI data without collaboration of a motion capture system.

- We developed a computer-assisted visualization system to provide 3D visualization of ROM.

- We designed and simulated three different kinds of hip joint movements including plane-movements, successive movements and combinational movement. Also, we experimented on these movements with envelope visualization.

- We evaluated the effect of moving center on the ROM in the relation to mobility of the joint.

Our system may be useful

- For pre-operative computer simulation of FAI surgery. Expected-after-surgery-bone shape can be simulated in advance to check if ROM would be improved after surgery.

- To help a surgeon to communicate with patients by visualization of ROM graph and envelope.

- To encourage people by showing the maximum achievable ROM to train more their muscle and to lose fat. Our system examines ROMs only from bony structure of the 
joint isolating the effects of the connective soft tissues, since they are variable and can be altered through physical training.

- To prevent athletes, dancers or other people from having worn-out bone by repeated exercise to the direction of bone contact.

\subsection{Future Research}

There are still some open issues and research directions that can be pursued to enhance obtained results and the performance of proposed system. The following points can be taken into consideration when we decide to further develop the work accomplished in this thesis.

- More data should be collected to be able to analyze and compare the envelopes of ROMs for healthy hips with patients diagnosed with FAI, as well as, to compare the ROMs of the hip joint of different ages and races.

- The system has ability to integrate the cartilage information; at this research, we considered a uniform cartilage thickness between femur and acetabulum while it is variable. Therefore, more research needs to be done in the area of cartilage segmentation and modeling.

- ROM of patients can be collected from motion capture system or through physical examination to be able to compare those ROMs with the ROMs which are calculated with our proposed system from MRI of the patient. 


\section{References}

[1] R. Ganz, J. Parvizi, M. Beck, M. Leunig, H. Nötzli, and K. A. Siebenrock, "Femoroacetabular impingement: a cause for osteoarthritis of the hip," Clinical orthopaedics and related research, vol. 417, pp. 112-120, 2003.

[2] C. W. Pfirrmann, B. Mengiardi, C. Dora, F. Kalberer, M. Zanetti, and J. Hodler, "Cam and Pincer Femoroacetabular Impingement: Characteristic MR Arthrographic Findings in 50 Patients1," Radiology, vol. 240, pp. 778-785, 2006.

[3] M. Beck, M. Kalhor, M. Leunig, and R. Ganz, "Hip morphology influences the pattern of damage to the acetabular cartilage FEMOROACETABULAR IMPINGEMENT AS A CAUSE OF EARLY OSTEOARTHRITIS OF THE HIP," Journal of Bone \& Joint Surgery, British Volume, vol. 87, pp. 1012-1018, 2005.

[4] C. Charbonnier, F. C. Kolo, V. B. Duthon, N. Magnenat-Thalmann, C. D. Becker, P. Hoffmeyer, et al., "Assessment of Congruence and Impingement of the Hip Joint in Professional Ballet Dancers A Motion Capture Study," The American journal of sports medicine, vol. 39, pp. 557-566, 2011.

[5] C. Charbonnier, N. Magnenat-Thalmann, C. D. Becker, P. Hoffmeyer, and J. Menetrey, "An integrated platform for hip joint osteoarthritis analysis: design, implementation and results," International journal of computer assisted radiology and surgery, vol. 5, pp. $351-358,2010$. 
[6] T. C. Chang, H. Kang, L. Arata, and W. Zhao, "A pre-operative approach of range of motion simulation and verification for femoroacetabular impingement," The International Journal of Medical Robotics and Computer Assisted Surgery, vol. 7, pp. 318-326, 2011.

[7] D. J. Berry and J. Lieberman, Surgery of the Hip vol. 2. Elsevier Health Sciences, 2012.

[8] K. Ashwell, The Anatomy Student's Self-Test Visual Dictionary: An All-in-One Anatomy Reference and Study Aid. Barron's Educational Series, 2011

[9] M. E. Madden, Introduction to Sectional Anatomy, 2 ed. Wolters Kluwer Health: Lippincott Williams \& Wilkins, 2008.

[10] D. P. Byrne, K. J. Mulhall, and J. F. Baker, "Anatomy \& biomechanics of the hip," Open Sports Medicine Journal, vol. 4, pp. 51-57, 2010.

[11] R. C. France, Introduction to Physical Education and Sports Science: CengageBrain. com, 2008.

[12] P. Baerlocher and R. Boulic, "Parametrization and range of motion of the ball-and-socket joint," in Deformable avatars, ed: Springer, 2001, pp. 180-190.

[13] J. Dario-Becker, Derived copy of Anatomy \& Physiology: A\&P II: Connexions 2013.

[14] M. H. M. L. a. A. Moroz. ( 2009). Physical Therapy (PT): Rehabilitation: Merck Manual Professional. Available: http://www.merck.com/mmpe/sec22/ch336/ch336b.html

[15] B. Mackenzie. (2004). Range of Movement (ROM). Available: http://www.brianmac.co.uk/musrom.htm

[16] D. Reynolds, J. Lucas, and K. Klaue, "Retroversion of the acetabulum a cause of hip pain," Journal of Bone \& Joint Surgery, British Volume, vol. 81, pp. 281-288, 1999. 
[17] M. Tannast, K. A. Siebenrock, and S. E. Anderson, "Femoroacetabular impingement: radiographic diagnosis — what the radiologist should know," American Journal of Roentgenology, vol. 188, pp. 1540-1552, 2007.

[18] M. JÄger, A. Wild, B. Westhoff, and R. Krauspe, "Femoroacetabular impingement caused by a femoral osseous head-neck bump deformity: clinical, radiological, and experimental results," Journal of Orthopaedic Science, vol. 9, pp. 256-263, 2004.

[19] A. Kassarjian, M. Brisson, and W. E. Palmer, "Femoroacetabular impingement," European journal of radiology, vol. 63, pp. 29-35, 2007.

[20] M. Leunig, W. J. Robertson, and R. Ganz, "Femoroacetabular impingement: diagnosis and management, including open surgical technique," Operative Techniques in Sports Medicine, vol. 15, pp. 178-188, 2007.

[21] M. Lavigne, J. Parvizi, M. Beck, K. A. Siebenrock, R. Ganz, and M. Leunig, "Anterior femoroacetabular impingement: part I. Techniques of joint preserving surgery," Clinical orthopaedics and related research, vol. 418, pp. 61-66, 2004.

[22] K. Siebenrock, R. Schoeniger, and R. Ganz, "Anterior Femoro-Acetabular Impingement Due to Acetabular Retroversion Treatment with Periacetabular Osteotomy," The Journal of Bone \& Joint Surgery, vol. 85, pp. 278-286, 2003.

[23] T. Sampson, "Arthroscopic treatment of femoroacetabular impingement: a proposed technique with clinical experience," Instructional course lectures, vol. 55, p. 337, 2006.

[24] V. M. Ilizaliturri Jr, "Complications of arthroscopic femoroacetabular impingement treatment: a review," Clinical orthopaedics and related research, vol. 467, pp. 760-768, 2009. 
[25] M. Teschner, J. Richolt, R. Kikinis, and B. Girod, "Computer-assisted analysis of hip joint flexibility," Proc. of Image and Multidimensional Digital Signal Processing IMDSP, vol. 98, pp. 63-66, 1998.

[26] S. J. Piazza, A. Erdemir, N. Okita, and P. R. Cavanagh, "Assessment of the functional method of hip joint center location subject to reduced range of hip motion," Journal of Biomechanics, vol. 37, pp. 349-356, 2004.

[27] M. Puls, T. M. Ecker, M. Tannast, S. D. Steppacher, K. A. Siebenrock, and J. H. Kowal, "The Equidistant Method-a novel hip joint simulation algorithm for detection of femoroacetabular impingement," Computer Aided Surgery, vol. 15, pp. 75-82, 2010.

[28] V. Camomilla, A. Cereatti, G. Vannozzi, and A. Cappozzo, "An optimized protocol for hip joint centre determination using the functional method," Journal of biomechanics, vol. 39, pp. 1096-1106, 2006.

[29] R. A. Siston and S. L. Delp, "Evaluation of a new algorithm to determine the hip joint center," Journal of biomechanics, vol. 39, pp. 125-130, 2006.

[30] M. Kennedy, M. Lamontagne, and P. Beaule, "The effect of cam femoroacetabular impingement on hip maximal dynamic range of motion," Journal of Orthopedics, vol. 1, pp. 41-50, 2009.

[31] M. Kubiak-Langer, M. Tannast, S. Murphy, K. Siebenrock, and F. Langlotz, "Range of motion in anterior femoroacetabular impingement," Clinical orthopaedics and related research, vol. 458, pp. 117-124, 2007.

[32] M. Kang, H. Sadri, L. Moccozet, N. Magnenat-Thalmann, and P. Hoffmeyer, "Accurate simulation of hip joint range of motion," in Computer Animation, 2002. Proceedings of, 2002, pp. 215-219. 
[33] D. Cai, W.-S. Lee, C. Joslin, and P. Beaulé, "Rapid Impingement Detection System with Uniform Sampling for Ball-and-Socket Joint," in Recent Advances in the $3 D$ Physiological Human, ed: Springer, 2009, pp. 179-192.

[34] A. Maciel, R. Boulic, and D. Thalmann, "Efficient collision detection within deforming spherical sliding contact," Visualization and Computer Graphics, IEEE Transactions on, vol. 13, pp. 518-529, 2007.

[35] E. Arbabi, R. Boulic, and D. Thalmann, "Fast collision detection methods for joint surfaces," Journal of biomechanics, vol. 42, pp. 91-99, 2009.

[36] M. Yazdifar, M. Yazdifar, P. Rahmanivahid, S. Eshraghi, I. Esat, and M. Chizari, "Evaluation of Hip Impingement Kinematics on Range of Motion," in Human-Computer Interaction. Towards Intelligent and Implicit Interaction, ed: Springer, 2013, pp. 262269.

[37] A. Leardini, A. Cappozzo, F. Catani, S. Toksvig-Larsen, A. Petitto, V. Sforza, et al., "Validation of a functional method for the estimation of hip joint centre location," Journal of biomechanics, vol. 32, pp. 99-103, 1999.

[38] A. L. Bell, R. A. Brand, and D. R. Pedersen, "Prediction of hip joint centre location from external landmarks," Human Movement Science, vol. 8, pp. 3-16, 1989.

[39] A. L. Bell, D. R. Pedersen, and R. A. Brand, "A comparison of the accuracy of several hip center location prediction methods," Journal of biomechanics, vol. 23, pp. 617-621, 1990.

[40] M. Cao, C. Ye, O. Doessel, and C. Liu, "Spherical parameter detection based on hierarchical Hough transform," Pattern recognition letters, vol. 27, pp. 980-986, 2006. 
[41] R. Suppanee, M. Yazdifar, M. Chizari, I. Esat, N. V. Bardakos, and R. E. Field, "Simulating osteoarthritis: the effect of the changing thickness of articular cartilage on the kinematics and pathological bone-to-bone contact in a hip joint with femoroacetabular impingement," European Orthopaedics and Traumatology, pp. 1-9, 2013.

[42] J. Illingworth and J. Kittler, "A survey of the Hough transform," Computer vision, graphics, and image processing, vol. 44, pp. 87-116, 1988.

[43] V. Leavers, "Which hough transform?," CVGIP: Image understanding, vol. 58, pp. 250264, 1993.

[44] M. Kang, "Hip joint center location by fitting conchoid shape to the acetabular rim region of MR images," in Engineering in Medicine and Biology Society, 2004. IEMBS'04. 26th Annual International Conference of the IEEE, 2004, pp. 4477-4480.

[45] F. Menschik, "The hip joint as a conchoid shape," Journal of biomechanics, vol. 30, pp. 971-973, 1997.

[46] S. Chegini, M. Beck, and S. J. Ferguson, "The effects of impingement and dysplasia on stress distributions in the hip joint during sitting and walking: a finite element analysis," Journal of Orthopaedic Research, vol. 27, pp. 195-201, 2009.

[47] B. Gilles, L. Moccozet, and N. Magnenat-Thalmann, "Anatomical modelling of the musculoskeletal system from MRI," in Medical Image Computing and ComputerAssisted Intervention-MICCAI 2006, ed: Springer, 2006, pp. 289-296.

[48] M. Kang, H. Sadri, L. Moccozet, and N. Magnenat-Thalmann, "Hip joint modeling for the control of the joint center and the range of motions," in Proceedings of the IFAC symposium on modelling and control in biomedical systems, 2003, pp. 23-27. 
[49] E. Arbabi, R. Boulic, and D. Thalmann, "A fast method for finding range of motion in the human joints," in Engineering in Medicine and Biology Society, 2007. EMBS 2007. 29th Annual International Conference of the IEEE, 2007, pp. 5079-5082.

[50] S. Kockara, T. Halic, K. Iqbal, C. Bayrak, and R. Rowe, "Collision detection: A survey," in Systems, Man and Cybernetics, 2007. ISIC. IEEE International Conference on, 2007, pp. 4046-4051.

[51] M. Teschner, S. Kimmerle, B. Heidelberger, G. Zachmann, L. Raghupathi, A. Fuhrmann, et al., "Collision detection for deformable objects," in Computer Graphics Forum, 2005, pp. 61-81.

[52] T. Larsson and T. Akenine-Möller, "Collision detection for continuously deforming bodies," 2001.

[53] M. Teschner, B. Heidelberger, M. Müller, D. Pomerantes, and M. H. Gross, "Optimized Spatial Hashing for Collision Detection of Deformable Objects," in VMV, 2003, pp. 4754.

[54] M. Eitz and G. Lixu, "Hierarchical spatial hashing for real-time collision detection," in Shape Modeling and Applications, 2007. SMI'07. IEEE International Conference on, 2007, pp. 61-70.

[55] Y. Kim, S. O. Koo, D. Lee, L. Kim, and S. Park, "Mesh-to-mesh collision detection by ray tracing for medical simulation with deformable bodies," in Cyberworlds (CW), 2010 International Conference on, 2010, pp. 60-66.

[56] K. E. Hoff III, J. Keyser, M. Lin, D. Manocha, and T. Culver, "Fast computation of generalized Voronoi diagrams using graphics hardware," in Proceedings of the 26th annual conference on Computer graphics and interactive techniques, 1999, pp. 277-286. 
[57] R. Barzel, J. R. Hughes, and D. N. Wood, "Plausible motion simulation for computer graphics animation," in Computer Animation and Simulation'96, ed: Springer, 1996, pp. 183-197.

[58] G. v. d. Bergen, "Efficient collision detection of complex deformable models using AABB trees," Journal of Graphics Tools, vol. 2, pp. 1-13, 1997.

[59] S. Gottschalk, M. C. Lin, and D. Manocha, "OBBTree: a hierarchical structure for rapid interference detection," in Proceedings of the 23rd annual conference on Computer graphics and interactive techniques, 1996, pp. 171-180.

[60] J. T. Klosowski, M. Held, J. S. Mitchell, H. Sowizral, and K. Zikan, "Efficient collision detection using bounding volume hierarchies of k-DOPs," Visualization and Computer Graphics, IEEE Transactions on, vol. 4, pp. 21-36, 1998.

[61] G. Zachmann, "Rapid collision detection by dynamically aligned DOP-trees," in Virtual Reality Annual International Symposium, 1998. Proceedings., IEEE 1998, 1998, pp. 9097.

[62] S. A. Ehmann and M. C. Lin, "Accurate and fast proximity queries between polyhedra using convex surface decomposition," in Computer Graphics Forum, 2001, pp. 500-511.

[63] S. Fisher and M. C. Lin, "Deformed distance fields for simulation of non-penetrating flexible bodies," in Computer Animation and Simulation 2001, ed: Springer, 2001, pp. $99-111$.

[64] D. E. Breen, S. Mauch, R. T. Whitaker, and J. Mao, "3d metamorphosis between different types of geometric models," in Computer Graphics Forum, 2001, pp. 36-48. 
[65] R. Bridson, S. Marino, and R. Fedkiw, "Simulation of clothing with folds and wrinkles," in Proceedings of the 2003 ACM SIGGRAPH/Eurographics symposium on Computer animation, 2003, pp. 28-36.

[66] S. F. Frisken, R. N. Perry, A. P. Rockwood, and T. R. Jones, "Adaptively sampled distance fields: a general representation of shape for computer graphics," in Proceedings of the 27th annual conference on Computer graphics and interactive techniques, 2000, pp. 249-254.

[67] J. Wu and L. Kobbelt, "Piecewise Linear Approximation of Signed Distance Fields," in $V M V, 2003$, pp. 513-520.

[68] D. Knott, "Cinder: Collision and interference detection in real-time using graphics hardware," 2003.

[69] R. Krug, A. J. Burghardt, S. Majumdar, and T. M. Link, "High-resolution imaging techniques for the assessment of osteoporosis," Radiologic clinics of North America, vol. 48, p. 601, 2010.

[70] H. Gray and W. H. Lewis, Anatomy of the Human Body: Lea \& Febiger, 1918.

[71] F. Bernardini, J. Mittleman, H. Rushmeier, C. Silva, and G. Taubin, "The ball-pivoting algorithm for surface reconstruction," Visualization and Computer Graphics, IEEE Transactions on, vol. 5, pp. 349-359, 1999.

[72] M. Attene, "A lightweight approach to repairing digitized polygon meshes," The Visual Computer, vol. 26, pp. 1393-1406, 2010.

[73] M. Sik and J. Krivánek, "Fast Random Sampling of Triangular Meshes," in Pacific Graphics Short Papers, 2013, pp. 17-22. 


\section{Publication by author}

1. Sahar Aghayan, WonSook Lee, "Calculation and Visualization of Range of Motion of Hip Joint from MRI", accepted to 27TH IEEE INTERNATIONAL SYMPOSIUM ON COMPUTER-BASED MEDICAL SYSTEMS (CBMS 2014), May 27, 2014 - May 29, 2014, New York, USA 